\title{
Air quality improvement in a megacity: implications from 2015 Beijing Parade Blue pollution control actions
}

Wen Xu et al.

Correspondence to: Xuejun Liu (liu310@cau.edu.cn)

The copyright of individual parts of the supplement might differ from the CC-BY 3.0 licence. 
1 Supporting Information (SI)

2 Figure captions

3 Figure S1. Pictures showing the sites \#15 (a) and \#20 (b) on the $5^{\text {th }}$ ring road, site \#2

4 on the $3^{\text {rd }}$ ring road (c) and site $\# 10$ on the $4^{\text {th }}$ ring road (d).

5 Figure S2. Correlations between daily average concentrations of $\mathrm{PM}_{2.5}$ with those of

$6 \mathrm{CO}(\mathrm{a}), \mathrm{NO}_{2}(\mathrm{~b}), \mathrm{SO}_{2}(\mathrm{c}), \mathrm{CO}+\mathrm{NO}_{2}+\mathrm{SO}_{2}(\mathrm{~d})$ and $\mathrm{CO}+\mathrm{NO}_{2}(\mathrm{e})$.

7 Figure S3. Map showing the distance between sampling site \#22 and the Wanliu

8 monitoring station in Beijing (http://map.baidu.com/).

9 Figure S4. Daily average sulfur oxidation ratio (SOR) and nitrogen oxidation ratio

10 (NOR) during the pre-Parade Blue, Parade Blue and post-Parade Blue periods.

11 Figure S5. Correlations between $\mathrm{NH}_{4}{ }^{+}$and $\mathrm{SO}_{4}{ }^{2-}, \mathrm{SO}_{4}{ }^{2-}+\mathrm{NO}_{3}{ }^{-}$(unit: $\mu \mathrm{eq} \mathrm{m}{ }^{-3}$ ) during 12 the pre-Parade Blue, Parade Blue and post-Parade Blue periods. 

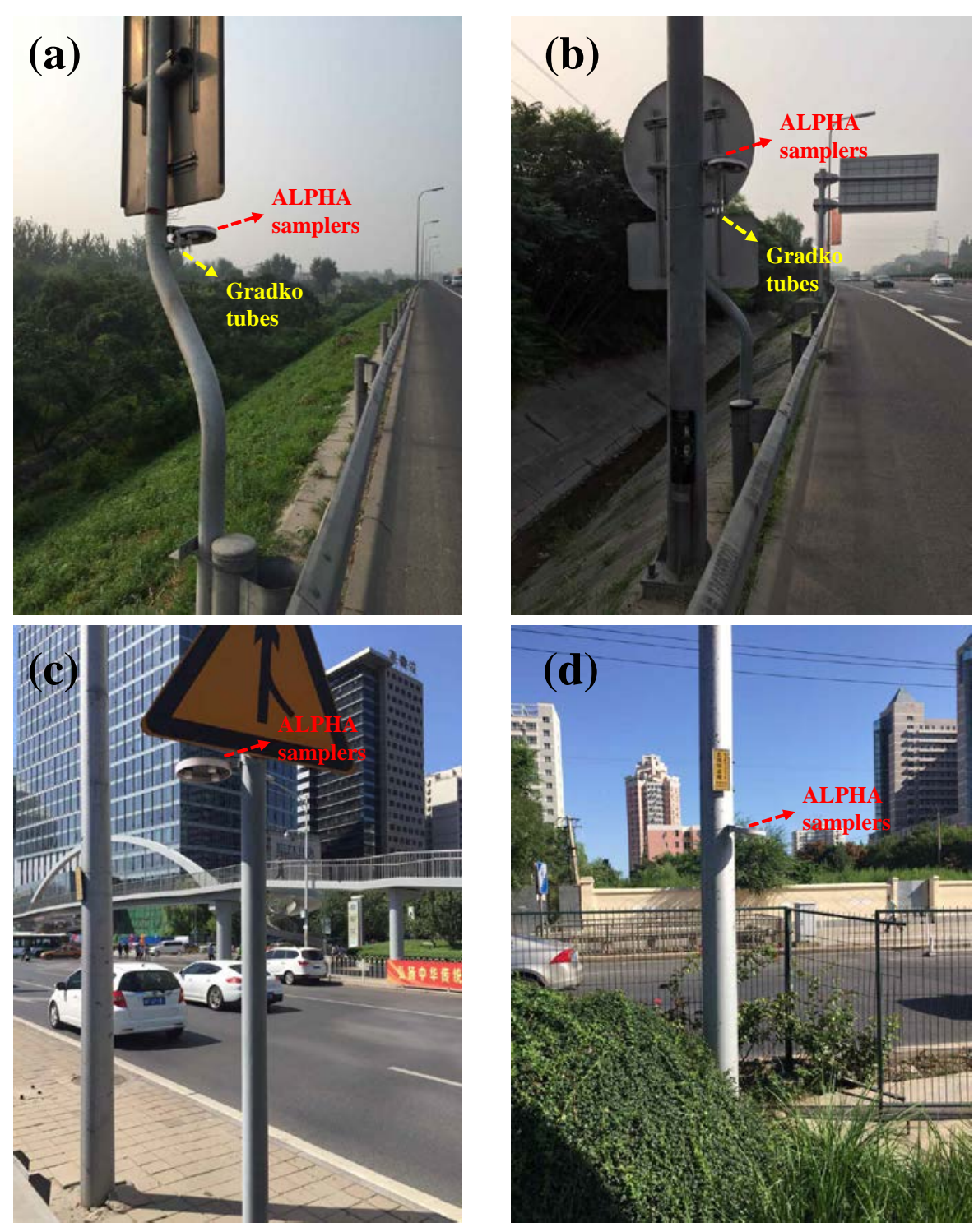

32 

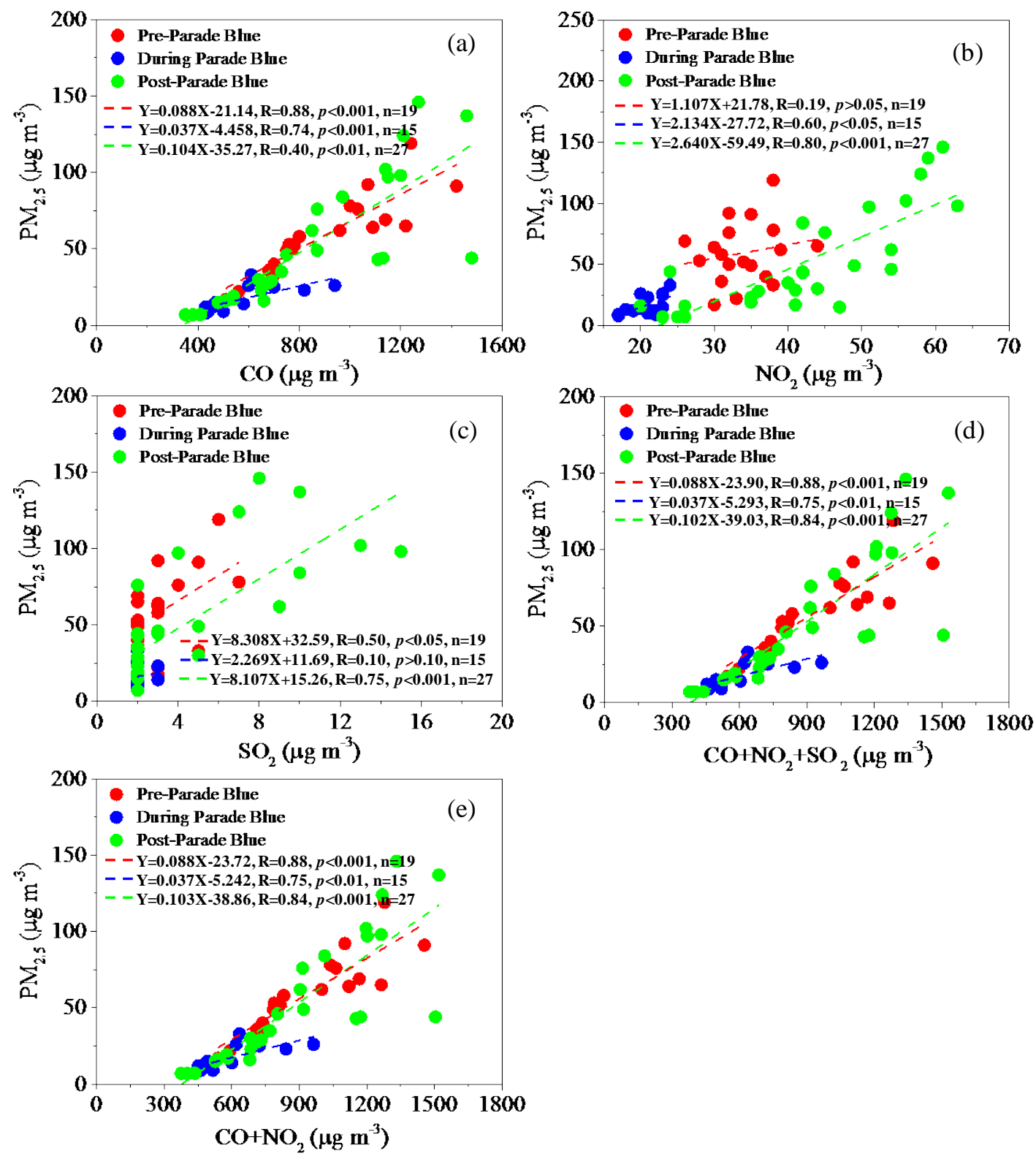
Figure S3

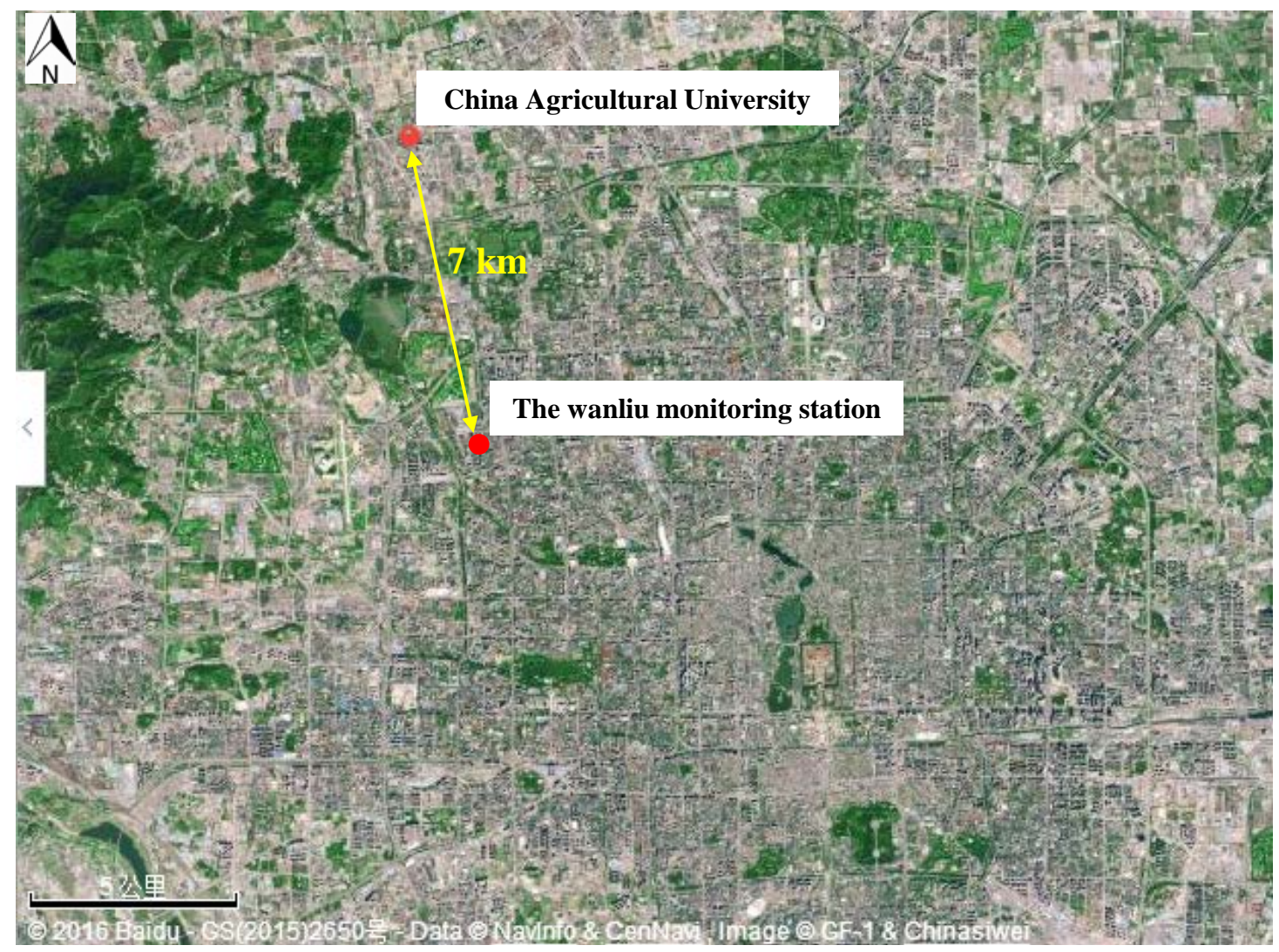

58

59

60

61

62

63

64

65

66

67

68

69

70

71

72

73 
76

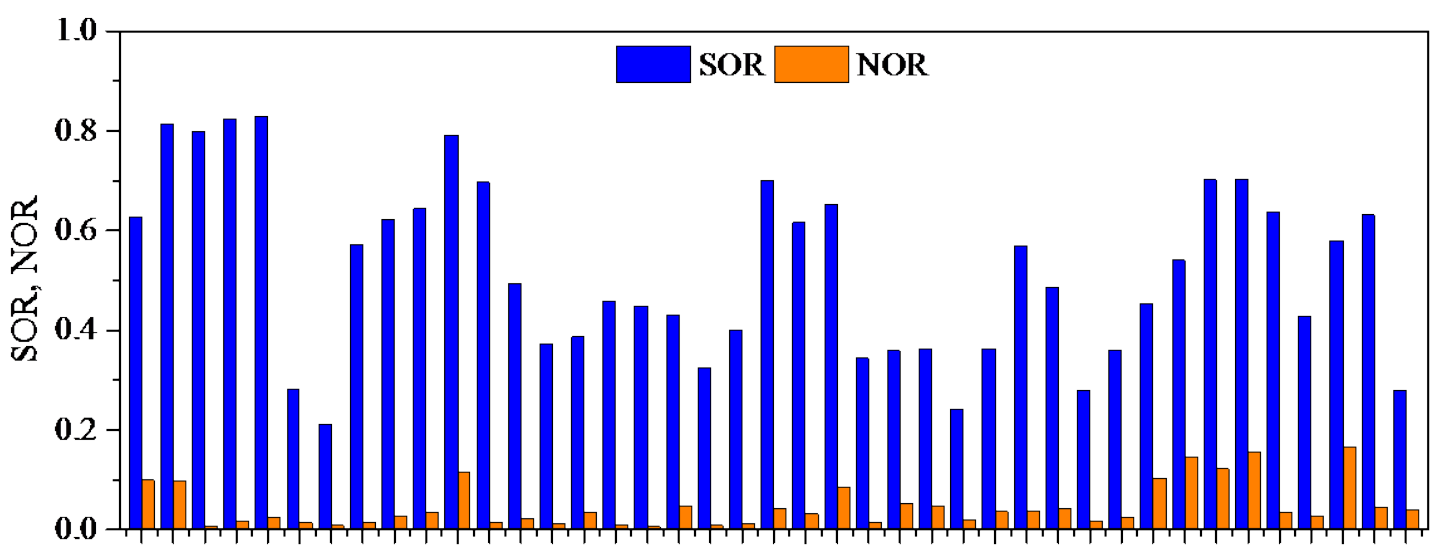

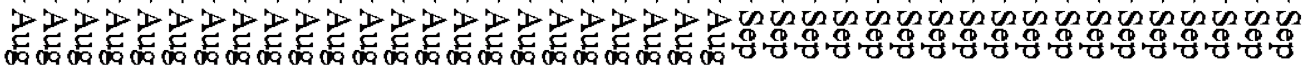
WのV 

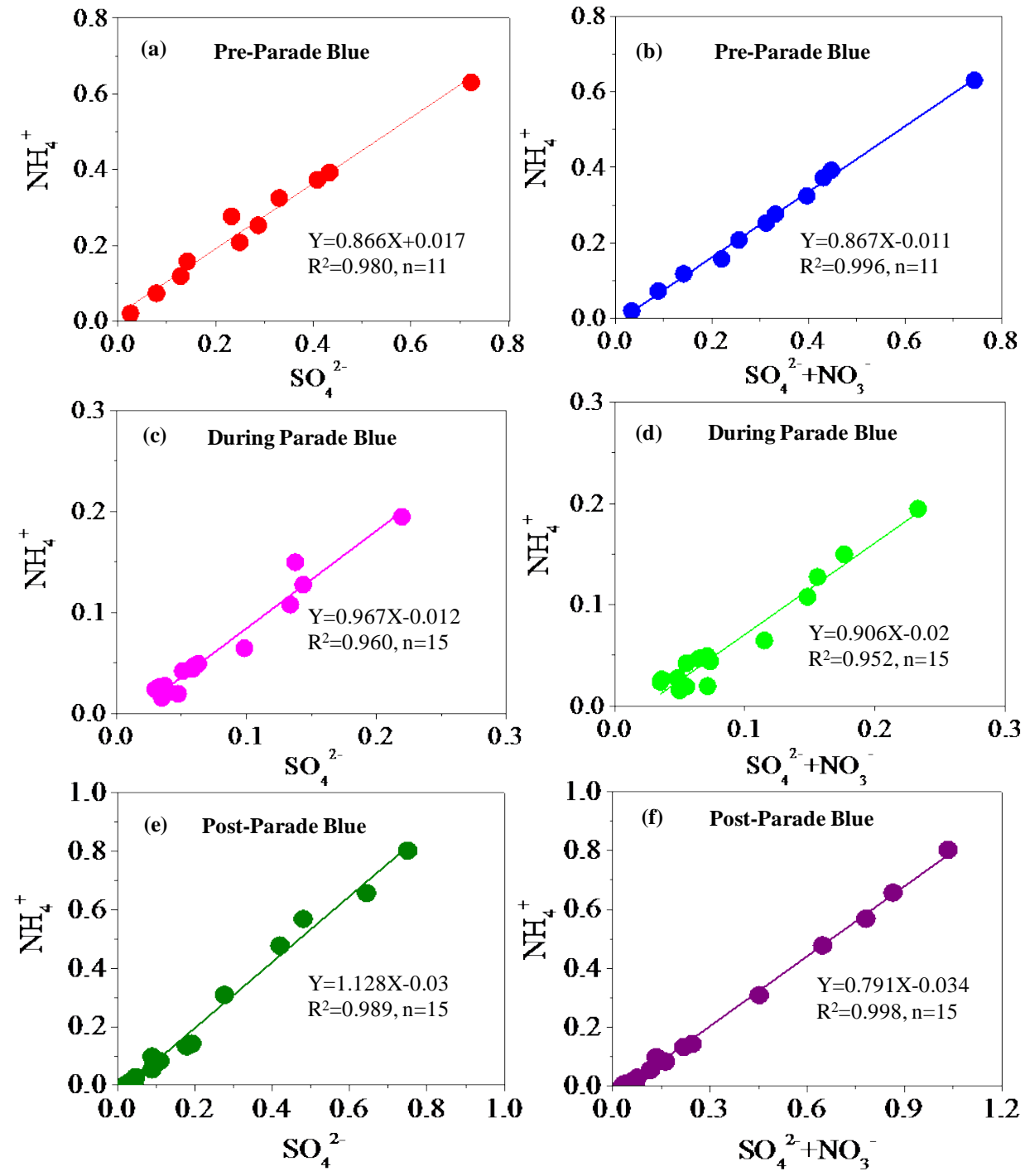

89

90

91

92

93

94

95

96

97

98

99 


\section{Figure S6}
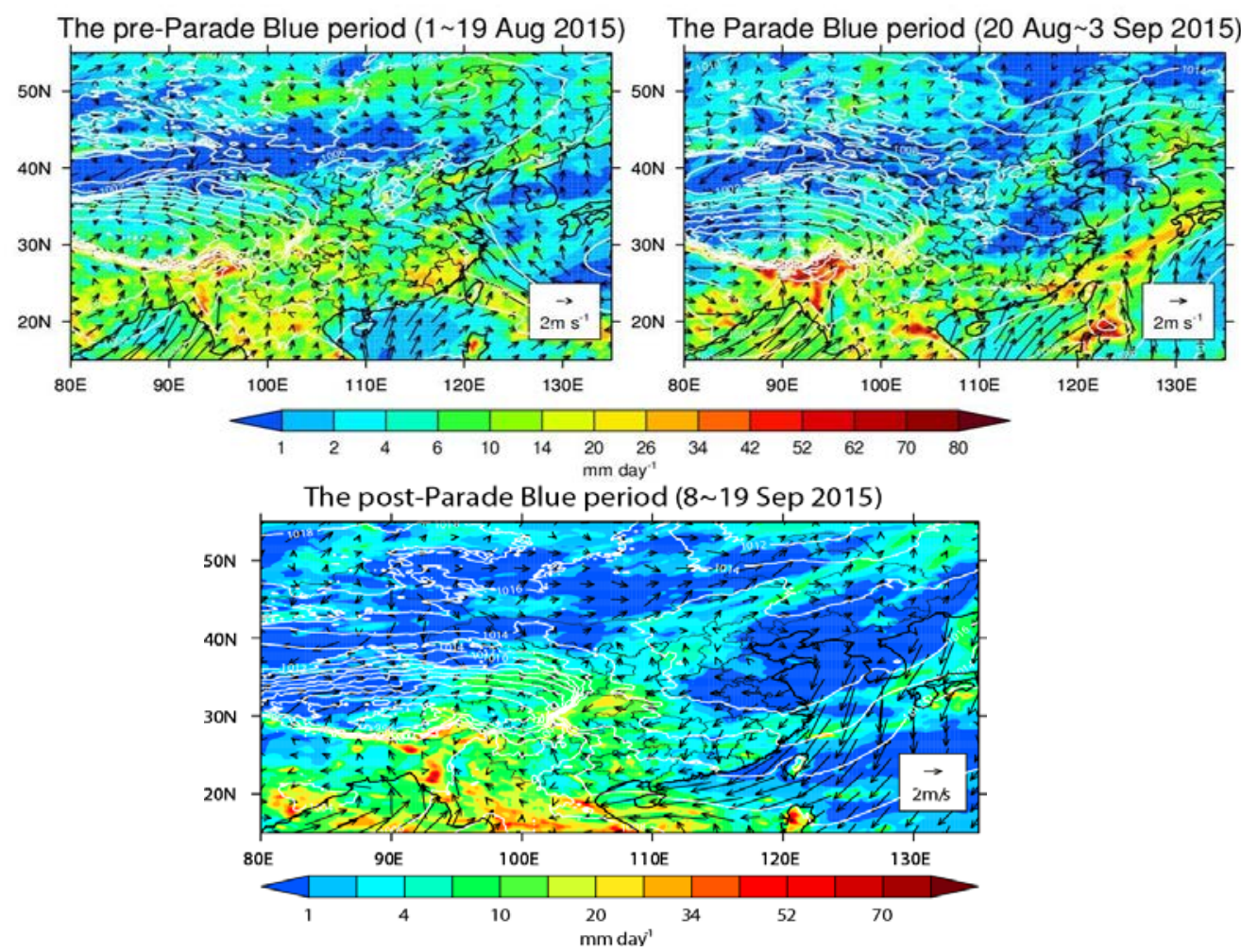
Table S1. Information on the thirty-one monitoring sites.

\begin{tabular}{|c|c|c|c|c|c|c|c|c|c|c|c|}
\hline \multirow[t]{2}{*}{$\begin{array}{l}\text { Site } \\
\text { code }\end{array}$} & \multirow[t]{2}{*}{ Coordinate } & \multirow[t]{2}{*}{ Sampling place } & \multirow[t]{2}{*}{$\begin{array}{l}\text { Site } \\
\text { type }^{a}\end{array}$} & \multirow[t]{2}{*}{$\begin{array}{l}\text { Measured } \\
\text { pollutants }\end{array}$} & \multicolumn{3}{|c|}{$\begin{array}{l}\text { Sampling durations } \\
\text { for each pollutant }\end{array}$} & \multicolumn{3}{|c|}{ Numbers of samples ${ }^{c}$} & \multirow{2}{*}{$\begin{array}{l}\text { Surrounding } \\
\text { environment and } \\
\text { possible emission } \\
\text { sources }\end{array}$} \\
\hline & & & & & $\mathrm{NH}_{3}$ & $\mathrm{NO}_{2}$ & $\mathrm{PM}_{2.5}$ & $\mathrm{NH}_{3}$ & $\mathrm{NO}_{2}$ & $\mathrm{PM}_{2.5}$ & \\
\hline 1 & $116.37^{\circ} \mathrm{E}, 39.95^{\circ} \mathrm{N}$ & $\begin{array}{l}\text { At Beijing Normal } \\
\text { University }\end{array}$ & NRS & $\mathrm{NH}_{3}$ & II, III & n.m. & n.m. & 2 & n.d. & n.d. & $\begin{array}{l}\text { Densely occupied } \\
\text { residences, } \\
\text { traffic roads }\end{array}$ \\
\hline 2 & $116.47^{\circ} \mathrm{E}, 39.91^{\circ} \mathrm{N}$ & On the $3^{\text {rd }}$ ring road & RS & $\mathrm{NH}_{3}$ & II, III & n.m. & n.m. & 2 & n.d. & n.d. & Traffic roads \\
\hline 3 & $116.39^{\circ} \mathrm{E}, 39.86^{\circ} \mathrm{N}$ & On the $3^{\text {rd }}$ ring road & RS & $\mathrm{NH}_{3}$ & II, III & n.m. & n.m. & 2 & n.d. & n.d. & Traffic roads \\
\hline 4 & $116.32^{\circ} \mathrm{E}, 39.92^{\circ} \mathrm{N}$ & On the $3^{\text {rd }}$ ring road & RS & $\mathrm{NH}_{3}$ & II, III & n.m. & n.m. & 2 & n.d. & n.d. & Traffic roads \\
\hline 5 & $116.38^{\circ} \mathrm{E}, 39.97^{\circ} \mathrm{N}$ & On the $3^{\text {rd }}$ ring road & RS & $\mathrm{NH}_{3}$ & II, III & n.m. & n.m. & 2 & n.d. & n.d. & Traffic roads \\
\hline 6 & $116.29^{\circ} \mathrm{E}, 39.95^{\circ} \mathrm{N}$ & $\begin{array}{l}\text { At Beijing } \\
\text { Academy of } \\
\text { Agricultural and } \\
\text { Forestry Sciences }\end{array}$ & NRS & $\mathrm{NH}_{3}$ & II, III & n.m. & n.m. & 2 & n.d. & n.d. & $\begin{array}{l}\text { Occupied residences, } \\
\text { traffic roads }\end{array}$ \\
\hline 7 & $116.36^{\circ} \mathrm{E}, 39.99^{\circ} \mathrm{N}$ & On the $4^{\text {th }}$ ring road & RS & $\mathrm{NH}_{3}$ & II, III & n.m. & n.m. & 2 & n.d. & n.d. & Traffic roads \\
\hline 8 & $116.28^{\circ} \mathrm{E}, 39.91^{\circ} \mathrm{N}$ & On the $4^{\text {th }}$ ring road & RS & $\mathrm{NH}_{3}$ & II, III & n.m. & n.m. & 2 & n.d. & n.d. & Traffic roads \\
\hline 9 & $116.39^{\circ} \mathrm{E}, 39.84^{\circ} \mathrm{N}$ & On the $4^{\text {th }}$ ring road & RS & $\mathrm{NH}_{3}$ & II, III & n.m. & n.m. & 2 & n.d. & n.d. & Traffic roads \\
\hline 10 & $116.49^{\circ} \mathrm{E}, 39.92^{\circ} \mathrm{N}$ & On the $4^{\text {th }}$ ring road & RS & $\mathrm{NH}_{3}$ & II, III & n.m. & n.m. & 2 & n.d. & n.d. & Traffic roads \\
\hline 11 & $116.39^{\circ} \mathrm{E}, 40.01^{\circ} \mathrm{N}$ & $\begin{array}{l}\text { Inside the Olympic } \\
\text { Park }\end{array}$ & NRS & $\mathrm{NH}_{3}$ & II, III & n.m. & n.m. & 2 & n.d. & n.d. & $\begin{array}{l}\text { Occupied residences, } \\
\text { traffic roads }\end{array}$ \\
\hline 12 & $116.54^{\circ} \mathrm{E}, 39.92^{\circ} \mathrm{N}$ & $\begin{array}{l}\text { Outside a sewage } \\
\text { treatment plant }\end{array}$ & NRS & $\mathrm{NH}_{3}$ & II, III & n.m. & n.m. & 2 & n.d. & n.d. & $\begin{array}{l}\text { Small villages, } \\
\text { sewage treatment } \\
\text { plant }\end{array}$ \\
\hline 13 & $116.29^{\circ} \mathrm{E}, 40.00^{\circ} \mathrm{N}$ & $\begin{array}{l}\text { Inside Summer } \\
\text { Palace }\end{array}$ & NRS & $\mathrm{NH}_{3}$ & II, III & n.m. & n.m. & 2 & n.d. & n.d. & $\begin{array}{l}\text { Occupied residences, } \\
\text { traffic roads }\end{array}$ \\
\hline
\end{tabular}




\begin{tabular}{|c|c|c|c|c|c|c|c|c|c|c|c|}
\hline 14 & $116.22^{\circ} \mathrm{E}, 39.99^{\circ} \mathrm{N}$ & On the $5^{\text {th }}$ ring road & RS & $\mathrm{NH}_{3}, \mathrm{NO}_{2}$ & $\begin{array}{l}\text { I, II, } \\
\text { III }\end{array}$ & $\begin{array}{l}\text { I, II, } \\
\text { III }\end{array}$ & n.m. & 3 & 3 & n.d. & Traffic roads \\
\hline 15 & $116.22^{\circ} \mathrm{E}, 39.91^{\circ} \mathrm{N}$ & On the $5^{\text {th }}$ ring road & RS & $\mathrm{NH}_{3}, \mathrm{NO}_{2}$ & $\begin{array}{l}\text { I, II, } \\
\text { III }\end{array}$ & $\begin{array}{l}\text { I, II, } \\
\text { III }\end{array}$ & n.m. & 3 & 3 & n.d. & Traffic roads \\
\hline 16 & $116.27^{\circ} \mathrm{E}, 39.79^{\circ} \mathrm{N}$ & On the $5^{\text {th }}$ ring road & RS & $\mathrm{NH}_{3}, \mathrm{NO}_{2}$ & $\begin{array}{l}\text { I, II, } \\
\text { III }\end{array}$ & $\begin{array}{l}\text { I, II, } \\
\text { III }\end{array}$ & n.m. & 3 & 3 & n.d. & Traffic roads \\
\hline 17 & $116.40^{\circ} \mathrm{E}, 39.77^{\circ} \mathrm{N}$ & On the $5^{\text {th }}$ ring road & RS & $\mathrm{NH}_{3}, \mathrm{NO}_{2}$ & $\begin{array}{l}\text { I, II, } \\
\text { III }\end{array}$ & $\begin{array}{l}\text { I, II, } \\
\text { III }\end{array}$ & n.m. & 3 & 3 & n.d. & Traffic roads \\
\hline 18 & $116.49^{\circ} \mathrm{E}, 39.81^{\circ} \mathrm{N}$ & On the $5^{\text {th }}$ ring road & RS & $\mathrm{NH}_{3}, \mathrm{NO}_{2}$ & $\begin{array}{l}\text { I, II, } \\
\text { III }\end{array}$ & $\begin{array}{l}\text { I, II, } \\
\text { III }\end{array}$ & n.m. & 3 & 3 & n.d. & Traffic roads \\
\hline 19 & $116.56^{\circ} \mathrm{E}, 39.88^{\circ} \mathrm{N}$ & On the $5^{\text {th }}$ ring road & RS & $\mathrm{NH}_{3}, \mathrm{NO}_{2}$ & $\begin{array}{l}\text { I, II, } \\
\text { III }\end{array}$ & $\begin{array}{l}\text { I, II, } \\
\text { III }\end{array}$ & n.m. & 3 & 3 & n.d. & Traffic roads \\
\hline 20 & $116.52^{\circ} \mathrm{E}, 39.99^{\circ} \mathrm{N}$ & On the $5^{\text {th }}$ ring road & RS & $\mathrm{NH}_{3}, \mathrm{NO}_{2}$ & $\begin{array}{l}\text { I, II, } \\
\text { III }\end{array}$ & $\begin{array}{l}\text { I, II, } \\
\text { III }\end{array}$ & n.m. & 3 & 3 & n.d. & Traffic roads \\
\hline 21 & $116.39^{\circ} \mathrm{E}, 40.03^{\circ} \mathrm{N}$ & On the $5^{\text {th }}$ ring road & RS & $\mathrm{NH}_{3}, \mathrm{NO}_{2}$ & $\begin{array}{l}\text { I, II, } \\
\text { III }\end{array}$ & $\begin{array}{l}\text { I, II, } \\
\text { III }\end{array}$ & n.m. & 3 & 3 & n.d. & Traffic roads \\
\hline 22 & $116.29^{\circ} \mathrm{E}, 40.03^{\circ} \mathrm{N}$ & $\begin{array}{l}\text { At China } \\
\text { Agricultural } \\
\text { University }\end{array}$ & NRS & $\begin{array}{l}\mathrm{NH}_{3}, \mathrm{NO}_{2}, \\
\mathrm{PM}_{2.5}\end{array}$ & $\begin{array}{l}\text { I, II, } \\
\text { III }\end{array}$ & $\begin{array}{l}\text { I, II, } \\
\text { III }\end{array}$ & $\begin{array}{l}\text { I, II, } \\
\text { IV }\end{array}$ & 3 & 3 & 41 & $\begin{array}{l}\text { Densely occupied } \\
\text { residences, industry, } \\
\text { small-scale urban } \\
\text { agriculture, and } \\
\text { traffic roads }\end{array}$ \\
\hline 23 & $116.26^{\circ} \mathrm{E}, 40.04^{\circ} \mathrm{N}$ & $\begin{array}{l}\text { On the Baiwang } \\
\text { hill }\end{array}$ & NRS & $\mathrm{NH}_{3}, \mathrm{NO}_{2}$ & $\begin{array}{l}\text { I, II, } \\
\text { III }\end{array}$ & $\begin{array}{l}\text { I, II, } \\
\text { III }\end{array}$ & n.m. & 3 & 3 & n.d. & $\begin{array}{l}\text { Small villages and } \\
\text { roads }\end{array}$ \\
\hline 24 & $116.23^{\circ} \mathrm{E}, 40.07^{\circ} \mathrm{N}$ & At a landfill site & NRS & $\mathrm{NH}_{3}$ & II, III & n.m. & n.m. & 2 & n.d. & n.d. & $\begin{array}{l}\text { Small villages, } \\
\text { refuse landfill }\end{array}$ \\
\hline 25 & $116.19^{\circ} \mathrm{E}, 40.14^{\circ} \mathrm{N}$ & $\begin{array}{l}\text { In Shangzhuang } \\
\text { Experimental } \\
\text { Station }\end{array}$ & NRS & $\mathrm{NH}_{3}, \mathrm{NO}_{2}$ & $\begin{array}{l}\text { I, II, } \\
\text { III }\end{array}$ & $\begin{array}{l}\text { I, II, } \\
\text { III }\end{array}$ & n.m. & 3 & 3 & n.d. & $\begin{array}{l}\text { Small villages, } \\
\text { croplands }\end{array}$ \\
\hline 26 & $116.14^{\circ} \mathrm{E}, 40.26^{\circ} \mathrm{N}$ & On an expressway & RS & $\mathrm{NH}_{3}, \mathrm{NO}_{2}$ & II, III & $\begin{array}{l}\text { II, } \\
\text { III }\end{array}$ & n.m. & 2 & 2 & n.d. & Traffic roads \\
\hline
\end{tabular}




\begin{tabular}{|c|c|c|c|c|c|c|c|c|c|c|c|}
\hline 27 & $116.02^{\circ} \mathrm{E}, 40.36^{\circ} \mathrm{N}$ & $\begin{array}{l}\text { Inside Badaling } \\
\text { Highway Tunnel }\end{array}$ & RS & $\mathrm{NH}_{3}, \mathrm{NO}_{2}$ & II, III & $\begin{array}{l}\text { II, } \\
\text { III }\end{array}$ & n.m. & 2 & 2 & n.d. & Traffic roads \\
\hline 28 & $116.01^{\circ} \mathrm{E}, 40.36^{\circ} \mathrm{N}$ & $\begin{array}{l}\text { Near the exit of } \\
\text { Badaling Highway } \\
\text { Tunnel }\end{array}$ & RS & $\mathrm{NH}_{3}, \mathrm{NO}_{2}$ & II, III & $\begin{array}{l}\text { II, } \\
\text { III }\end{array}$ & n.m. & 2 & 2 & n.d. & Traffic roads \\
\hline 29 & $116.58^{\circ} \mathrm{E}, 36.84^{\circ} \mathrm{N}$ & $\begin{array}{l}\text { In Yucheng } \\
\text { Experimental } \\
\text { Station }\end{array}$ & RS & $\begin{array}{l}\mathrm{NH}_{3}, \mathrm{NO}_{2}, \\
\mathrm{PM}_{2.5}\end{array}$ & $\begin{array}{l}\text { I, II, } \\
\text { III }\end{array}$ & $\begin{array}{l}\text { I, II, } \\
\text { III }\end{array}$ & $\begin{array}{l}\text { I, II, } \\
\text { IV }\end{array}$ & 3 & 3 & 21 & $\begin{array}{l}\text { Small villages, } \\
\text { croplands }\end{array}$ \\
\hline 30 & $115.02^{\circ} \mathrm{E}, 36.87^{\circ} \mathrm{N}$ & $\begin{array}{l}\text { In Quzhou } \\
\text { Experimental } \\
\text { Station }\end{array}$ & RS & $\begin{array}{l}\mathrm{NH}_{3}, \mathrm{NO}_{2}, \\
\mathrm{PM}_{2.5}\end{array}$ & $\begin{array}{l}\text { I, II, } \\
\text { III }\end{array}$ & $\begin{array}{l}\text { I, II, } \\
\text { III }\end{array}$ & $\begin{array}{l}\text { I, II, } \\
\text { IV }\end{array}$ & 3 & 3 & 19 & $\begin{array}{l}\text { Small villages, } \\
\text { croplands }\end{array}$ \\
\hline 31 & $\begin{array}{l}120.75^{\circ} \mathrm{E}, 37.93^{\circ} \\
\mathrm{N}\end{array}$ & $\begin{array}{l}\text { On Changdao } \\
\text { Island }\end{array}$ & BS & $\mathrm{NH}_{3}, \mathrm{NO}_{2}$ & $\begin{array}{l}\text { I, II, } \\
\text { III }\end{array}$ & $\begin{array}{l}\text { I, II, } \\
\text { III }\end{array}$ & n.m. & 3 & 3 & n.d. & $\begin{array}{l}\text { Almost no } \\
\text { agricultural and } \\
\text { industrial activities }\end{array}$ \\
\hline
\end{tabular}

${ }^{\mathrm{a}} \mathrm{NRS}$, RS and BS denote non-road site, road site and background site, respectively.

${ }^{\text {b }}$ I, II, III, and IV denote the periods of 3-19 August 2015, 20 August-3 September 2015, 8-19 September 2015, and 4-30 September 2015, respectively; n.m. means no measurements.

${ }^{\mathrm{d}}$ Values for $\mathrm{NH}_{3}$ and $\mathrm{NO}_{2}$ samples multiply by 3 to obtain the total numbers of samples; n.d. means no data. 
116 Table S2. Summary of daily average $\mathrm{PM}_{2.5}$ concentrations $\left(\mu \mathrm{g} \mathrm{m}^{-3}\right)$ during the pre-Parade Blue, Parade Blue and post-Parade Blue periods in the 117291 cities across China.

\begin{tabular}{|c|c|c|c|c|c|c|c|c|c|c|c|c|c|c|}
\hline \multirow{2}{*}{ City } & \multirow{2}{*}{ Province } & \multirow{2}{*}{ Region $^{\mathrm{a}}$} & \multicolumn{4}{|c|}{ Pre-Parade Blue period ${ }^{\mathrm{b}}$} & \multicolumn{4}{|c|}{ Parade Blue period $^{\mathrm{b}}$} & \multicolumn{4}{|c|}{ Post-Parade Blue period $^{\mathrm{b}}$} \\
\hline & & & Mean & SE & Min & Max & Mean & $\mathrm{SE}$ & Min & Max & Mean & $\mathrm{SE}$ & Min & Max \\
\hline Beijing & Municipality & EC & 59 & 6 & 17 & 119 & 17 & 2 & 8 & 33 & 52 & 8 & 7 & 146 \\
\hline Tianjing & Municipality & $\mathrm{EC}$ & 62 & 5 & 20 & 116 & 32 & 4 & 15 & 77 & 47 & 5 & 9 & 116 \\
\hline Baoding & Hebei & $\mathrm{EC}$ & 88 & 7 & 29 & 148 & 35 & 4 & 16 & 75 & 66 & 8 & 15 & 159 \\
\hline Cangzhou & Hebei & EC & 59 & 6 & 17 & 119 & 17 & 2 & 8 & 33 & 39 & 5 & 9 & 88 \\
\hline Chengde & Hebei & $\mathrm{EC}$ & 31 & 4 & 11 & 73 & 14 & 0 & 11 & 17 & 28 & 4 & 9 & 95 \\
\hline Handan & Hebei & EC & 81 & 7 & 35 & 147 & 52 & 7 & 9 & 91 & 63 & 6 & 15 & 131 \\
\hline Hengshui & Hebei & $\mathrm{EC}$ & 91 & 7 & 33 & 159 & 47 & 5 & 25 & 92 & 76 & 8 & 24 & 167 \\
\hline Langfang & Hebei & $\mathrm{EC}$ & 69 & 6 & 17 & 113 & 24 & 4 & 5 & 78 & 54 & 7 & 4 & 132 \\
\hline Qinhuangdao & Hebei & $\mathrm{EC}$ & 45 & 6 & 10 & 92 & 7 & 1 & 4 & 23 & 15 & 2 & 4 & 46 \\
\hline Shijiazhuang & Hebei & $\mathrm{EC}$ & 78 & 7 & 20 & 128 & 35 & 5 & 6 & 77 & 45 & 5 & 11 & 98 \\
\hline Tangshan & Hebei & $\mathrm{EC}$ & 73 & 6 & 25 & 118 & 13 & 2 & 4 & 29 & 43 & 5 & 8 & 91 \\
\hline Xingtai & Hebei & $\mathrm{EC}$ & 83 & 7 & 30 & 144 & 45 & 7 & 8 & 101 & 60 & 5 & 17 & 103 \\
\hline Zhangjiakou & Hebei & $\mathrm{EC}$ & 29 & 3 & 17 & 66 & 18 & 1 & 9 & 27 & 27 & 4 & 11 & 71 \\
\hline Changzhi & Shanxi & $\mathrm{EC}$ & 59 & 5 & 22 & 96 & 39 & 3 & 25 & 55 & 47 & 3 & 15 & 79 \\
\hline Datong & Shanxi & $\mathrm{EC}$ & 26 & 2 & 13 & 59 & 16 & 1 & 12 & 22 & 27 & 3 & 9 & 71 \\
\hline Jincheng & Shanxi & EC & 41 & 3 & 11 & 69 & 37 & 3 & 19 & 70 & 37 & 4 & 8 & 104 \\
\hline Jinzhong & Shanxi & EC & 39 & 3 & 21 & 71 & 26 & 3 & 11 & 51 & 43 & 4 & 8 & 88 \\
\hline Linfen & Shanxi & $\mathrm{EC}$ & 32 & 2 & 19 & 44 & 27 & 2 & 17 & 43 & 40 & 3 & 11 & 63 \\
\hline Lvliang & Shanxi & $\mathrm{EC}$ & 46 & 4 & 17 & 88 & 29 & 2 & 18 & 40 & 46 & 4 & 18 & 85 \\
\hline Shuozhou & Shanxi & $\mathrm{EC}$ & 39 & 2 & 18 & 54 & 28 & 1 & 22 & 36 & 39 & 4 & 17 & 89 \\
\hline Taiyuan & Shanxi & $\mathrm{EC}$ & 44 & 3 & 26 & 73 & 23 & 3 & 9 & 55 & 46 & 5 & 10 & 106 \\
\hline
\end{tabular}




\begin{tabular}{|c|c|c|c|c|c|c|c|c|c|c|c|c|c|c|}
\hline Xinzhou & Shanxi & $\mathrm{EC}$ & 39 & 3 & 23 & 61 & 20 & 2 & 12 & 36 & 44 & 5 & 12 & 107 \\
\hline Yangquan & Shanxi & $\mathrm{EC}$ & 38 & 3 & 16 & 64 & 19 & 2 & 9 & 31 & 36 & 4 & 6 & 70 \\
\hline Yuncheng & Shanxi & $\mathrm{EC}$ & 58 & 3 & 29 & 78 & 48 & 3 & 29 & 69 & 38 & 2 & 16 & 68 \\
\hline Binzhou & Shandong & $\mathrm{EC}$ & 49 & 5 & 12 & 94 & 37 & 4 & 18 & 69 & 51 & 6 & 12 & 139 \\
\hline Dezhou & Shandong & $\mathrm{EC}$ & 89 & 6 & 25 & 133 & 44 & 4 & 23 & 85 & 72 & 8 & 22 & 159 \\
\hline Dongying & Shandong & $\mathrm{EC}$ & 65 & 7 & 21 & 123 & 43 & 4 & 21 & 80 & 51 & 6 & 13 & 117 \\
\hline Jinan & Shandong & $\mathrm{EC}$ & 59 & 5 & 21 & 87 & 50 & 4 & 30 & 82 & 72 & 5 & 23 & 118 \\
\hline Jining & Shandong & $\mathrm{EC}$ & 50 & 5 & 22 & 103 & 49 & 3 & 34 & 78 & 58 & 4 & 21 & 101 \\
\hline Laiwu & Shandong & $\mathrm{EC}$ & 54 & 6 & 19 & 98 & 65 & 5 & 40 & 101 & 62 & 5 & 19 & 122 \\
\hline Liaocheng & Shandong & $\mathrm{EC}$ & 67 & 6 & 24 & 105 & 49 & 5 & 17 & 80 & 73 & 6 & 27 & 122 \\
\hline Linyi & Shandong & $\mathrm{EC}$ & 37 & 4 & 15 & 76 & 43 & 4 & 25 & 78 & 45 & 6 & 13 & 133 \\
\hline Qingdao & Shandong & $\mathrm{EC}$ & 35 & 4 & 9 & 60 & 30 & 3 & 14 & 71 & 25 & 3 & 9 & 71 \\
\hline Rizhao & Shandong & $\mathrm{EC}$ & 39 & 4 & 8 & 75 & 40 & 5 & 16 & 74 & 37 & 5 & 7 & 93 \\
\hline Tai'an & Shandong & $\mathrm{EC}$ & 42 & 4 & 21 & 76 & 40 & 3 & 20 & 67 & 51 & 4 & 20 & 92 \\
\hline Weihai & Shandong & $\mathrm{EC}$ & 38 & 4 & 19 & 74 & 22 & 2 & 7 & 31 & 20 & 2 & 7 & 47 \\
\hline Weifang & Shandong & $\mathrm{EC}$ & 51 & 6 & 17 & 102 & 55 & 7 & 25 & 115 & 50 & 7 & 17 & 170 \\
\hline Yantai & Shandong & $\mathrm{EC}$ & 39 & 5 & 14 & 87 & 23 & 2 & 9 & 36 & 23 & 2 & 9 & 63 \\
\hline Zaozhuang & Shandong & $\mathrm{EC}$ & 45 & 5 & 16 & 83 & 51 & 3 & 40 & 74 & 60 & 6 & 9 & 144 \\
\hline Zibo & Shandong & $\mathrm{EC}$ & 56 & 4 & 29 & 90 & 55 & 4 & 35 & 89 & 68 & 6 & 26 & 135 \\
\hline Alxa League & Inner Mongolia & $\mathrm{EC}$ & 38 & 3 & 18 & 66 & 33 & 1 & 22 & 40 & 36 & 2 & 21 & 60 \\
\hline Bayannur & Inner Mongolia & $\mathrm{EC}$ & 33 & 3 & 15 & 56 & 32 & 1 & 21 & 41 & 39 & 3 & 22 & 71 \\
\hline Baotou & Inner Mongolia & $\mathrm{EC}$ & 35 & 3 & 11 & 56 & 22 & 1 & 13 & 30 & 33 & 3 & 8 & 74 \\
\hline Chifeng & Inner Mongolia & $\mathrm{EC}$ & 22 & 2 & 10 & 47 & 13 & 1 & 11 & 18 & 25 & 3 & 7 & 69 \\
\hline Ordos & Inner Mongolia & $\mathrm{EC}$ & 22 & 3 & 5 & 40 & 12 & 1 & 8 & 18 & 22 & 3 & 6 & 61 \\
\hline Hohhot & Inner Mongolia & $\mathrm{EC}$ & 23 & 2 & 8 & 43 & 14 & 1 & 10 & 23 & 27 & 3 & 5 & 64 \\
\hline Hulun Buir & Inner Mongolia & $\mathrm{EC}$ & 28 & 2 & 18 & 48 & 26 & 2 & 16 & 38 & 22 & 1 & 15 & 36 \\
\hline
\end{tabular}




\begin{tabular}{|c|c|c|c|c|c|c|c|c|c|c|c|c|c|c|}
\hline Tongliao & Inner Mongolia & EC & 46 & 6 & 17 & 99 & 25 & 3 & 17 & 55 & 27 & 3 & 13 & 74 \\
\hline Wuhai & Inner Mongolia & EC & 42 & 4 & 18 & 85 & 30 & 2 & 18 & 44 & 51 & 5 & 18 & 109 \\
\hline Hinggan League & Inner Mongolia & $\mathrm{EC}$ & 27 & 2 & 15 & 37 & 21 & 1 & 15 & 28 & 18 & 1 & 8 & 33 \\
\hline Anyang & Henan & EC & 61 & 5 & 30 & 106 & 46 & 5 & 16 & 82 & 68 & 6 & 29 & 158 \\
\hline Hebi & Henan & EC & 50 & 4 & 26 & 76 & 31 & 4 & 10 & 61 & 44 & 4 & 20 & 92 \\
\hline Jiaozuo & Henan & EC & 40 & 3 & 13 & 61 & 41 & 4 & 12 & 63 & 37 & 3 & 15 & 70 \\
\hline Kaifeng & Henan & EC & 45 & 4 & 21 & 81 & 38 & 4 & 17 & 69 & 54 & 5 & 20 & 124 \\
\hline Luoyang & Henan & EC & 56 & 6 & 20 & 95 & 41 & 3 & 21 & 56 & 41 & 4 & 12 & 80 \\
\hline Luohe & Henan & EC & 48 & 5 & 21 & 87 & 52 & 3 & 31 & 71 & 81 & 7 & 32 & 194 \\
\hline Nanyang & Henan & EC & 49 & 4 & 22 & 84 & 56 & 4 & 31 & 85 & 55 & 4 & 33 & 97 \\
\hline Pingdingshan & Henan & EC & 53 & 6 & 19 & 91 & 50 & 3 & 33 & 73 & 68 & 5 & 29 & 142 \\
\hline Sanmenxia & Henan & EC & 58 & 5 & 17 & 94 & 39 & 3 & 20 & 56 & 36 & 3 & 8 & 66 \\
\hline Shangqiu & Henan & EC & 41 & 4 & 19 & 88 & 40 & 4 & 18 & 79 & 47 & 4 & 18 & 102 \\
\hline Xinyang & Henan & EC & 29 & 4 & 13 & 67 & 40 & 3 & 22 & 61 & 47 & 4 & 15 & 94 \\
\hline Zhengzhou & Henan & EC & 61 & 6 & 25 & 112 & 50 & 6 & 17 & 98 & 70 & 6 & 31 & 162 \\
\hline Zhoukou & Henan & EC & 36 & 3 & 22 & 60 & 42 & 3 & 21 & 58 & 68 & 6 & 26 & 146 \\
\hline Zhumadian & Henan & $\mathrm{EC}$ & 41 & 4 & 16 & 76 & 41 & 3 & 16 & 59 & 60 & 6 & 20 & 130 \\
\hline Anshan & Liaoning & NEC & 42 & 5 & 14 & 72 & 43 & 2 & 30 & 59 & 41 & 4 & 17 & 95 \\
\hline Benxi & Liaoning & NEC & 34 & 3 & 17 & 72 & 30 & 2 & 12 & 45 & 31 & 3 & 11 & 61 \\
\hline Chaoyang & Liaoning & NEC & 30 & 4 & 13 & 65 & 14 & 1 & 9 & 25 & 30 & 5 & 9 & 105 \\
\hline Dalian & Liaoning & NEC & 41 & 5 & 13 & 97 & 23 & 3 & 11 & 52 & 24 & 2 & 8 & 59 \\
\hline Dandong & Liaoning & NEC & 24 & 4 & 1 & 51 & 20 & 2 & 8 & 28 & 26 & 2 & 8 & 53 \\
\hline Fushun & Liaoning & NEC & 27 & 3 & 12 & 63 & 18 & 2 & 6 & 28 & 28 & 3 & 6 & 54 \\
\hline Fuxin & Liaoning & NEC & 44 & 6 & 11 & 97 & 18 & 2 & 9 & 34 & 32 & 3 & 10 & 83 \\
\hline Huludao & Liaoning & NEC & 66 & 9 & 20 & 137 & 24 & 2 & 15 & 48 & 34 & 4 & 9 & 92 \\
\hline Jinzhou & Liaoning & NEC & 58 & 8 & 23 & 125 & 19 & 2 & 10 & 44 & 34 & 4 & 10 & 85 \\
\hline
\end{tabular}




\begin{tabular}{|c|c|c|c|c|c|c|c|c|c|c|c|c|c|c|}
\hline Liaoyang & Liaoning & NEC & 36 & 4 & 11 & 69 & 31 & 2 & 18 & 41 & 34 & 3 & 11 & 80 \\
\hline Panjin & Liaoning & NEC & 41 & 6 & 13 & 93 & 18 & 2 & 10 & 43 & 26 & 3 & 7 & 59 \\
\hline Shenyang & Liaoning & NEC & 36 & 4 & 16 & 66 & 23 & 2 & 11 & 35 & 36 & 4 & 10 & 77 \\
\hline Tieling & Liaoning & NEC & 28 & 3 & 13 & 53 & 17 & 2 & 7 & 31 & 28 & 3 & 11 & 71 \\
\hline Wafangdian & Liaoning & NEC & 48 & 6 & 19 & 103 & 27 & 1 & 21 & 38 & 38 & 3 & 13 & 84 \\
\hline Yingkou & Liaoning & NEC & 25 & 3 & 11 & 45 & 23 & 3 & 9 & 51 & 22 & 3 & 5 & 66 \\
\hline Baicheng & Jilin & NEC & 47 & 2 & 36 & 63 & 42 & 1 & 32 & 51 & 36 & 2 & 22 & 61 \\
\hline Baishan & Jilin & NEC & 29 & 3 & 15 & 52 & 25 & 2 & 17 & 38 & 30 & 2 & 17 & 43 \\
\hline Changchun & Jilin & NEC & 29 & 4 & 16 & 73 & 23 & 2 & 8 & 39 & 27 & 2 & 12 & 63 \\
\hline Jilin & Jilin & NEC & 19 & 2 & 10 & 39 & 18 & 2 & 8 & 30 & 24 & 2 & 13 & 54 \\
\hline Liaoyuan & Jilin & NEC & 36 & 5 & 13 & 76 & 36 & 6 & 14 & 81 & 36 & 3 & 17 & 79 \\
\hline Siping & Jilin & NEC & 35 & 5 & 15 & 89 & 23 & 2 & 12 & 38 & 33 & 3 & 15 & 80 \\
\hline Songyuan & Jilin & NEC & 20 & 1 & 10 & 36 & 13 & 1 & 5 & 23 & 19 & 2 & 8 & 41 \\
\hline Daqing & Heilongjiang & NEC & 30 & 2 & 17 & 51 & 21 & 1 & 15 & 33 & 22 & 2 & 13 & 42 \\
\hline Daxinganling & Heilongjiang & NEC & 13 & 2 & 5 & 40 & 10 & 1 & 7 & 14 & 13 & 1 & 4 & 29 \\
\hline Harbin & Heilongjiang & NEC & 25 & 2 & 15 & 38 & 18 & 2 & 11 & 35 & 21 & 2 & 10 & 42 \\
\hline Hegang & Heilongjiang & NEC & 47 & 2 & 33 & 70 & 28 & 4 & 6 & 61 & 11 & 1 & 5 & 27 \\
\hline Heihe & Heilongjiang & NEC & 33 & 1 & 26 & 44 & 27 & 1 & 18 & 34 & 10 & 1 & 4 & 33 \\
\hline Jixi & Heilongjiang & NEC & 19 & 1 & 14 & 25 & 16 & 1 & 11 & 27 & 19 & 1 & 9 & 32 \\
\hline Jiamusi & Heilongjiang & NEC & 15 & 1 & 6 & 24 & 13 & 1 & 7 & 29 & 11 & 1 & 5 & 17 \\
\hline Mudanjiang & Heilongjiang & NEC & 20 & 1 & 16 & 27 & 22 & 1 & 14 & 33 & 23 & 1 & 16 & 38 \\
\hline Qitaihe & Heilongjiang & NEC & 22 & 1 & 17 & 32 & 21 & 1 & 14 & 33 & 25 & 1 & 13 & 39 \\
\hline Qiqihar & Heilongjiang & NEC & 19 & 2 & 7 & 31 & 13 & 1 & 6 & 22 & 16 & 1 & 6 & 31 \\
\hline Shuangyashan & Heilongjiang & NEC & 23 & 1 & 17 & 28 & 26 & 1 & 19 & 34 & 20 & 1 & 10 & 31 \\
\hline Suihua & Heilongjiang & NEC & 15 & 1 & 9 & 26 & 11 & 1 & 8 & 15 & 16 & 1 & 10 & 29 \\
\hline Ankang & Shaanxi & NEC & 21 & 2 & 7 & 43 & 27 & 2 & 13 & 44 & 37 & 3 & 13 & 63 \\
\hline
\end{tabular}




\begin{tabular}{|c|c|c|c|c|c|c|c|c|c|c|c|c|c|c|}
\hline Baoji & Shaanxi & NEC & 38 & 5 & 11 & 78 & 34 & 4 & 11 & 68 & 35 & 4 & 8 & 71 \\
\hline Hanzhong & Shaanxi & NEC & 27 & 3 & 10 & 60 & 32 & 2 & 18 & 40 & 32 & 2 & 9 & 58 \\
\hline Shangluo & Shaanxi & NEC & 28 & 3 & 9 & 65 & 27 & 2 & 14 & 42 & 35 & 3 & 12 & 65 \\
\hline Tongchuan & Shaanxi & NEC & 36 & 4 & 12 & 71 & 32 & 2 & 14 & 45 & 37 & 3 & 11 & 60 \\
\hline Weinan & Shaanxi & NEC & 36 & 3 & 8 & 58 & 34 & 3 & 17 & 52 & 55 & 5 & 10 & 119 \\
\hline Xi'an & Shaanxi & NEC & 45 & 4 & 13 & 73 & 40 & 3 & 20 & 66 & 38 & 3 & 11 & 77 \\
\hline Xianyang & Shaanxi & NEC & 38 & 4 & 10 & 60 & 35 & 3 & 19 & 55 & 43 & 4 & 14 & 94 \\
\hline Yan'an & Shaanxi & NEC & 33 & 3 & 14 & 59 & 24 & 2 & 14 & 44 & 36 & 4 & 12 & 70 \\
\hline Yulin & Shaanxi & NEC & 30 & 3 & 12 & 53 & 20 & 2 & 9 & 33 & 31 & 3 & 5 & 75 \\
\hline Baiying & Gansu & NEC & 26 & 3 & 9 & 65 & 27 & 1 & 21 & 33 & 26 & 2 & 9 & 47 \\
\hline Dingxi & Gansu & NEC & 21 & 1 & 9 & 29 & 28 & 1 & 19 & 36 & 22 & 1 & 14 & 32 \\
\hline Gannan & Gansu & NEC & 17 & 1 & 9 & 33 & 26 & 3 & 15 & 53 & 21 & 2 & 9 & 42 \\
\hline Jiayuguan & Gansu & NEC & 23 & 5 & 10 & 86 & 26 & 3 & 12 & 58 & 17 & 2 & 7 & 48 \\
\hline Jiuquan & Gansu & NEC & 36 & 6 & 19 & 121 & 35 & 3 & 18 & 61 & 26 & 1 & 17 & 51 \\
\hline Lanzhou & Gansu & NEC & 36 & 2 & 19 & 62 & 32 & 1 & 24 & 38 & 35 & 2 & 18 & 57 \\
\hline Linxia & Gansu & NEC & 18 & 1 & 7 & 28 & 21 & 2 & 12 & 35 & 23 & 1 & 13 & 31 \\
\hline Pingliang & Gansu & NEC & 30 & 3 & 10 & 68 & 20 & 2 & 12 & 29 & 23 & 2 & 10 & 55 \\
\hline Qingyang & Gansu & NEC & 22 & 3 & 7 & 57 & 20 & 2 & 11 & 31 & 20 & 2 & 5 & 39 \\
\hline Tianshui & Gansu & NEC & 18 & 3 & 6 & 67 & 14 & 2 & 6 & 30 & 11 & 1 & 4 & 22 \\
\hline Wuwei & Gansu & NEC & 27 & 4 & 14 & 77 & 27 & 2 & 11 & 44 & 19 & 1 & 8 & 32 \\
\hline Zhangye & Gansu & NEC & 21 & 2 & 9 & 54 & 17 & 2 & 5 & 30 & 17 & 1 & 10 & 44 \\
\hline Guyuan & Ningxia & NEC & 25 & 3 & 9 & 53 & 22 & 1 & 14 & 32 & 20 & 2 & 9 & 39 \\
\hline Shizuishan & Ningxia & NEC & 30 & 3 & 13 & 51 & 23 & 1 & 12 & 31 & 32 & 3 & 7 & 71 \\
\hline Wuzhong & Ningxia & NEC & 26 & 3 & 13 & 46 & 28 & 2 & 17 & 35 & 28 & 2 & 4 & 55 \\
\hline Yinchuan & Ningxia & NEC & 41 & 3 & 21 & 68 & 34 & 3 & 18 & 50 & 32 & 2 & 11 & 54 \\
\hline Zhongwei & Ningxia & NEC & 27 & 3 & 13 & 74 & 26 & 2 & 17 & 36 & 29 & 3 & 8 & 60 \\
\hline
\end{tabular}




\begin{tabular}{|c|c|c|c|c|c|c|c|c|c|c|c|c|c|c|}
\hline Aksu & Sinkiang & NEC & 99 & 16 & 22 & 281 & 52 & 6 & 28 & 95 & 43 & 6 & 18 & 169 \\
\hline Hami & Sinkiang & NEC & 29 & 9 & 11 & 191 & 22 & 2 & 11 & 40 & 18 & 1 & 9 & 36 \\
\hline Hotan & Sinkiang & NEC & 130 & 29 & 26 & 646 & 82 & 12 & 21 & 180 & 61 & 8 & 19 & 215 \\
\hline Kashgar & Sinkiang & NEC & 103 & 20 & 25 & 329 & 45 & 10 & 10 & 141 & 59 & 8 & 12 & 156 \\
\hline Karamay & Sinkiang & NEC & 20 & 1 & 15 & 35 & 16 & 1 & 12 & 20 & 16 & 1 & 10 & 26 \\
\hline Kizilsu & Sinkiang & NEC & 59 & 13 & 7 & 209 & 20 & 5 & 0 & 65 & 27 & 5 & 6 & 97 \\
\hline Shihezi & Sinkiang & NEC & 32 & 6 & 9 & 106 & 21 & 2 & 11 & 35 & 28 & 6 & 8 & 155 \\
\hline Urumchi & Sinkiang & NEC & 32 & 3 & 10 & 58 & 30 & 3 & 15 & 56 & 27 & 3 & 9 & 75 \\
\hline Wujiaqu & Sinkiang & NEC & 32 & 3 & 12 & 57 & 30 & 3 & 13 & 52 & 32 & 4 & 5 & 90 \\
\hline Yili & Sinkiang & NEC & 26 & 2 & 11 & 35 & 26 & 2 & 10 & 37 & 29 & 3 & 10 & 58 \\
\hline Ezhou & Hubei & NEC & 33 & 4 & 8 & 67 & 51 & 3 & 22 & 71 & 49 & 2 & 18 & 72 \\
\hline Huanggang & Hubei & NEC & 33 & 3 & 9 & 64 & 46 & 3 & 21 & 60 & 42 & 2 & 14 & 61 \\
\hline Jingmen & Hubei & NEC & 32 & 4 & 13 & 77 & 57 & 5 & 23 & 84 & 66 & 4 & 21 & 120 \\
\hline Shiyan & Hubei & NEC & 30 & 3 & 9 & 61 & 42 & 4 & 23 & 64 & 51 & 4 & 13 & 101 \\
\hline Wuhan & Hubei & NEC & 32 & 3 & 13 & 62 & 53 & 4 & 24 & 76 & 47 & 2 & 19 & 64 \\
\hline Xianning & Hubei & NEC & 31 & 3 & 10 & 62 & 57 & 3 & 25 & 69 & 44 & 2 & 18 & 61 \\
\hline Xiangyang & Hubei & NEC & 33 & 4 & 10 & 74 & 45 & 4 & 24 & 74 & 55 & 4 & 19 & 94 \\
\hline Xiaogan & Hubei & NEC & 36 & 3 & 19 & 61 & 57 & 3 & 31 & 76 & 53 & 3 & 30 & 85 \\
\hline Yichang & Hubei & NEC & 29 & 4 & 10 & 74 & 48 & 4 & 26 & 84 & 48 & 4 & 10 & 79 \\
\hline Anqing & Anhui & NEC & 38 & 3 & 14 & 58 & 35 & 3 & 14 & 58 & 39 & 2 & 11 & 57 \\
\hline Bengbu & Anhui & NEC & 41 & 4 & 12 & 71 & 55 & 4 & 18 & 76 & 52 & 4 & 13 & 103 \\
\hline Bozhou & Anhui & NEC & 34 & 3 & 19 & 63 & 63 & 5 & 28 & 103 & 61 & 4 & 16 & 114 \\
\hline Chuzhou & Anhui & NEC & 42 & 4 & 14 & 77 & 52 & 5 & 22 & 80 & 48 & 3 & 27 & 87 \\
\hline Fuyang & Anhui & NEC & 31 & 3 & 13 & 61 & 47 & 3 & 25 & 76 & 37 & 3 & 10 & 71 \\
\hline Hefei & Anhui & NEC & 35 & 4 & 8 & 60 & 43 & 4 & 13 & 65 & 40 & 3 & 15 & 63 \\
\hline Huaibei & Anhui & NEC & 37 & 4 & 14 & 86 & 41 & 2 & 22 & 51 & 45 & 4 & 16 & 85 \\
\hline
\end{tabular}




\begin{tabular}{|c|c|c|c|c|c|c|c|c|c|c|c|c|c|c|}
\hline Huainan & Anhui & NEC & 23 & 3 & 6 & 53 & 41 & 4 & 9 & 62 & 40 & 4 & 7 & 89 \\
\hline Huangshan & Anhui & NEC & 22 & 2 & 6 & 37 & 35 & 5 & 10 & 62 & 28 & 2 & 10 & 45 \\
\hline Lu'an & Anhui & NEC & 37 & 3 & 12 & 67 & 50 & 2 & 34 & 64 & 40 & 2 & 11 & 55 \\
\hline Ma'anshan & Anhui & NEC & 38 & 3 & 16 & 58 & 50 & 5 & 21 & 85 & 40 & 2 & 24 & 69 \\
\hline Suzhou & Anhui & NEC & 56 & 7 & 22 & 111 & 58 & 4 & 34 & 79 & 58 & 6 & 16 & 151 \\
\hline Tongling & Anhui & NEC & 42 & 4 & 14 & 80 & 54 & 5 & 24 & 85 & 34 & 2 & 19 & 51 \\
\hline Wuhu & Anhui & NEC & 30 & 3 & 10 & 52 & 44 & 4 & 17 & 70 & 41 & 3 & 21 & 68 \\
\hline Changzhou & Jiangsu & NEC & 31 & 3 & 11 & 67 & 40 & 5 & 8 & 61 & 29 & 2 & 16 & 63 \\
\hline Huaian & Jiangsu & NEC & 32 & 3 & 10 & 59 & 45 & 4 & 12 & 74 & 29 & 2 & 10 & 60 \\
\hline Lianyungang & Jiangsu & NEC & 33 & 4 & 9 & 64 & 42 & 5 & 13 & 90 & 31 & 4 & 6 & 85 \\
\hline Nanjing & Jiangsu & NEC & 29 & 3 & 7 & 47 & 39 & 4 & 13 & 63 & 28 & 2 & 14 & 63 \\
\hline Nantong & Jiangsu & NEC & 34 & 4 & 10 & 62 & 42 & 6 & 10 & 96 & 33 & 3 & 15 & 74 \\
\hline Suzhou & Jiangsu & NEC & 33 & 3 & 9 & 54 & 54 & 7 & 15 & 96 & 36 & 3 & 13 & 78 \\
\hline Suqian & Jiangsu & NEC & 37 & 3 & 12 & 67 & 45 & 3 & 18 & 71 & 37 & 4 & 8 & 103 \\
\hline Taizhou & Jiangsu & NEC & 36 & 4 & 10 & 68 & 38 & 4 & 13 & 62 & 34 & 3 & 16 & 64 \\
\hline Wuxi & Jiangsu & NEC & 35 & 3 & 12 & 59 & 52 & 6 & 15 & 90 & 38 & 2 & 18 & 77 \\
\hline Xuzhou & Jiangsu & NEC & 31 & 3 & 10 & 56 & 38 & 2 & 26 & 52 & 36 & 4 & 14 & 84 \\
\hline Yanchen & Jiangsu & NEC & 30 & 4 & 8 & 67 & 29 & 3 & 8 & 49 & 23 & 2 & 10 & 58 \\
\hline Yangzhou & Jiangsu & NEC & 31 & 3 & 9 & 57 & 38 & 3 & 17 & 55 & 30 & 3 & 11 & 67 \\
\hline Zhenjiang & Jiangsu & NEC & 38 & 4 & 10 & 65 & 46 & 5 & 15 & 70 & 37 & 3 & 17 & 80 \\
\hline Shanghai & Municipality & NEC & 31 & 3 & 9 & 54 & 56 & 8 & 11 & 122 & 30 & 2 & 10 & 71 \\
\hline Hangzhou & Zhejiang & NEC & 32 & 3 & 9 & 63 & 43 & 5 & 12 & 74 & 39 & 3 & 12 & 69 \\
\hline Huzhou & Zhejiang & NEC & 31 & 2 & 17 & 54 & 49 & 5 & 20 & 77 & 34 & 2 & 17 & 78 \\
\hline Jiaxing & Zhejiang & NEC & 32 & 3 & 10 & 47 & 52 & 6 & 16 & 99 & 37 & 3 & 13 & 85 \\
\hline Jinhua & Zhejiang & NEC & 33 & 3 & 13 & 57 & 53 & 6 & 19 & 100 & 47 & 4 & 16 & 93 \\
\hline Lishui & Zhejiang & NEC & 23 & 2 & 6 & 33 & 30 & 3 & 14 & 45 & 32 & 4 & 5 & 77 \\
\hline
\end{tabular}




\begin{tabular}{|c|c|c|c|c|c|c|c|c|c|c|c|c|c|c|}
\hline Ningbo & Zhejiang & NEC & 23 & 2 & 9 & 37 & 36 & 5 & 10 & 73 & 31 & 2 & 14 & 62 \\
\hline Quzhou & Zhejiang & NEC & 33 & 3 & 13 & 57 & 52 & 6 & 19 & 81 & 37 & 3 & 15 & 66 \\
\hline Shaoxing & Zhejiang & NEC & 33 & 3 & 12 & 63 & 57 & 7 & 21 & 113 & 43 & 3 & 11 & 70 \\
\hline Taizhou & Zhejiang & NEC & 25 & 2 & 10 & 40 & 29 & 4 & 8 & 61 & 30 & 2 & 16 & 67 \\
\hline Wenjiang & Zhejiang & NEC & 30 & 2 & 15 & 42 & 30 & 3 & 12 & 47 & 31 & 2 & 15 & 52 \\
\hline Zhoushan & Zhejiang & NEC & 17 & 1 & 8 & 30 & 23 & 3 & 7 & 47 & 19 & 2 & 11 & 48 \\
\hline Fuzhou & Jiangxi & NEC & 28 & 2 & 15 & 50 & 44 & 4 & 16 & 74 & 41 & 2 & 15 & 60 \\
\hline Ganzhou & Jiangxi & NEC & 38 & 3 & 20 & 63 & 44 & 4 & 24 & 73 & 44 & 2 & 28 & 67 \\
\hline Ji'an & Jiangxi & NEC & 32 & 3 & 15 & 56 & 41 & 4 & 19 & 65 & 43 & 2 & 18 & 61 \\
\hline Jingdezhen & Jiangxi & NEC & 31 & 2 & 14 & 47 & 45 & 4 & 17 & 66 & 32 & 2 & 13 & 54 \\
\hline Jiujiang & Jiangxi & NEC & 31 & 3 & 9 & 49 & 48 & 4 & 24 & 69 & 35 & 2 & 10 & 51 \\
\hline Nanchang & Jiangxi & NEC & 23 & 2 & 8 & 36 & 40 & 4 & 10 & 68 & 29 & 2 & 7 & 55 \\
\hline Pingxiang & Jiangxi & NEC & 23 & 2 & 13 & 40 & 49 & 5 & 15 & 78 & 44 & 3 & 18 & 62 \\
\hline Shangrao & Jiangxi & NEC & 41 & 3 & 19 & 61 & 38 & 4 & 20 & 71 & 32 & 2 & 17 & 65 \\
\hline Xinyu & Jiangxi & NEC & 25 & 2 & 10 & 36 & 38 & 4 & 15 & 59 & 34 & 2 & 11 & 47 \\
\hline Yichun & Jiangxi & NEC & 31 & 1 & 25 & 49 & 48 & 2 & 28 & 62 & 43 & 2 & 27 & 53 \\
\hline Yingtan & Jiangxi & NEC & 21 & 2 & 6 & 42 & 39 & 4 & 20 & 66 & 39 & 3 & 11 & 62 \\
\hline Changsha & Hunan & NEC & 27 & 2 & 14 & 49 & 59 & 4 & 21 & 86 & 48 & 3 & 15 & 89 \\
\hline Changde & Hunan & NEC & 22 & 2 & 6 & 43 & 31 & 2 & 11 & 43 & 32 & 2 & 9 & 56 \\
\hline Chenzhou & Hunan & NEC & 27 & 2 & 12 & 48 & 45 & 8 & 12 & 126 & 47 & 4 & 15 & 84 \\
\hline Huaihua & Hunan & NEC & 21 & 1 & 13 & 33 & 49 & 4 & 17 & 76 & 45 & 4 & 14 & 101 \\
\hline Loudi & Hunan & NEC & 28 & 2 & 15 & 40 & 54 & 3 & 21 & 71 & 44 & 3 & 15 & 90 \\
\hline Xiangtan & Hunan & NEC & 27 & 2 & 15 & 46 & 58 & 4 & 22 & 79 & 49 & 3 & 15 & 92 \\
\hline Yiyang & Hunan & NEC & 28 & 2 & 17 & 51 & 49 & 3 & 19 & 68 & 45 & 2 & 18 & 65 \\
\hline Yongzhou & Hunan & NEC & 37 & 2 & 25 & 55 & 44 & 3 & 22 & 63 & 54 & 5 & 24 & 133 \\
\hline Zhangjiajie & Hunan & NEC & 23 & 3 & 9 & 56 & 45 & 4 & 17 & 71 & 43 & 3 & 8 & 67 \\
\hline
\end{tabular}




\begin{tabular}{|c|c|c|c|c|c|c|c|c|c|c|c|c|c|}
\hline Zhuzhou & Hunan & NEC & 22 & 1 & 11 & 34 & 50 & 4 & 21 & 85 & 40 & 2 & 13 \\
\hline Dongguan & Guangdong & NEC & 26 & 2 & 11 & 45 & 35 & 3 & 16 & 57 & 39 & 2 & 19 \\
\hline Foshan & Guangdong & NEC & 34 & 3 & 20 & 59 & 44 & 4 & 18 & 75 & 38 & 2 & 17 \\
\hline Guangzhou & Guangdong & NEC & 34 & 2 & 17 & 47 & 39 & 3 & 19 & 63 & 41 & 2 & 23 \\
\hline Heyuan & Guangdong & NEC & 25 & 2 & 10 & 37 & 31 & 4 & 12 & 54 & 38 & 2 & 14 \\
\hline Huizhou & Guangdong & NEC & 22 & 2 & 10 & 39 & 25 & 4 & 7 & 44 & 24 & 2 & 6 \\
\hline Jiangmen & Guangdong & NEC & 19 & 2 & 10 & 39 & 36 & 5 & 12 & 67 & 33 & 2 & 15 \\
\hline Maoming & Guangdong & NEC & 19 & 3 & 5 & 56 & 28 & 6 & 4 & 65 & 25 & 3 & 9 \\
\hline Meizhou & Guangdong & NEC & 23 & 2 & 9 & 43 & 26 & 3 & 9 & 48 & 35 & 2 & 14 \\
\hline Qingyuan & Guangdong & NEC & 32 & 3 & 17 & 62 & 32 & 4 & 14 & 59 & 30 & 3 & 11 \\
\hline Shantou & Guangdong & NEC & 24 & 2 & 12 & 38 & 25 & 3 & 13 & 43 & 29 & 2 & 16 \\
\hline Shaoguan & Guangdong & NEC & 30 & 2 & 15 & 56 & 32 & 4 & 13 & 59 & 40 & 3 & 10 \\
\hline Shenzhen & Guangdong & NEC & 20 & 2 & 8 & 48 & 27 & 4 & 11 & 47 & 30 & 2 & 14 \\
\hline Zhujiang & Guangdong & NEC & 15 & 2 & 6 & 42 & 31 & 6 & 7 & 69 & 25 & 3 & 10 \\
\hline Zhaoqing & Guangdong & NEC & 30 & 2 & 15 & 53 & 40 & 4 & 17 & 73 & 40 & 3 & 16 \\
\hline Zhongshan & Guangdong & NEC & 19 & 3 & 7 & 56 & 28 & 5 & 7 & 59 & 31 & 3 & 11 \\
\hline Zhuhai & Guangdong & NEC & 17 & 3 & 6 & 55 & 26 & 5 & 7 & 57 & 26 & 2 & 9 \\
\hline Fuzhou & Fujian & NEC & 22 & 2 & 6 & 38 & 17 & 2 & 7 & 27 & 21 & 1 & 9 \\
\hline Longyan & Fujian & NEC & 17 & 2 & 5 & 36 & 19 & 2 & 10 & 37 & 27 & 2 & 11 \\
\hline Nanping & Fujian & NEC & 18 & 2 & 3 & 37 & 18 & 3 & 9 & 37 & 23 & 2 & 9 \\
\hline Ningde & Fujian & NEC & 21 & 2 & 7 & 35 & 17 & 2 & 7 & 30 & 21 & 1 & 8 \\
\hline Putian & Fujian & NEC & 23 & 2 & 10 & 44 & 19 & 2 & 9 & 30 & 25 & 1 & 11 \\
\hline Quanzhou & Fujian & NEC & 20 & 2 & 7 & 33 & 16 & 1 & 9 & 26 & 23 & 1 & 10 \\
\hline Sanming & Fujian & NEC & 21 & 2 & 4 & 38 & 22 & 3 & 11 & 44 & 25 & 2 & 7 \\
\hline Xiamen & Fujian & NEC & 21 & 2 & 10 & 36 & 19 & 1 & 12 & 30 & 27 & 1 & 11 \\
\hline Zhangzhou & Fujian & NEC & 20 & 2 & 8 & 44 & 20 & 1 & 14 & 29 & 26 & 1 & 9 \\
\hline
\end{tabular}




\begin{tabular}{|c|c|c|c|c|c|c|c|c|c|c|c|c|c|c|}
\hline Haikou & Hainan & NEC & 12 & 1 & 7 & 25 & 20 & 3 & 7 & 48 & 16 & 2 & 4 & 41 \\
\hline Sanya & Hainan & NEC & 10 & 0 & 6 & 13 & 11 & 1 & 6 & 19 & 12 & 1 & 4 & 21 \\
\hline Chongqing & Municipality & NEC & 34 & 3 & 11 & 64 & 53 & 3 & 24 & 71 & 30 & 3 & 10 & 61 \\
\hline Bazhong & Sichuan & NEC & 17 & 1 & 8 & 25 & 23 & 2 & 14 & 36 & 20 & 2 & 6 & 34 \\
\hline Chengdu & Sichuan & NEC & 33 & 4 & 9 & 75 & 54 & 4 & 30 & 78 & 31 & 3 & 9 & 88 \\
\hline Dazhou & Sichuan & NEC & 32 & 2 & 13 & 50 & 62 & 4 & 33 & 85 & 43 & 3 & 16 & 66 \\
\hline Deyang & Sichuan & NEC & 31 & 3 & 12 & 58 & 53 & 5 & 28 & 94 & 27 & 2 & 9 & 65 \\
\hline Guangyuan & Sichuan & NEC & 13 & 2 & 4 & 52 & 13 & 1 & 8 & 18 & 9 & 1 & 4 & 16 \\
\hline Leshan & Sichuan & NEC & 29 & 4 & 7 & 82 & 55 & 6 & 23 & 105 & 32 & 3 & 12 & 96 \\
\hline Luzhou & Sichuan & NEC & 36 & 5 & 6 & 84 & 57 & 8 & 13 & 111 & 27 & 2 & 9 & 52 \\
\hline Meishan & Sichuan & NEC & 32 & 4 & 8 & 63 & 51 & 6 & 25 & 100 & 28 & 4 & 8 & 117 \\
\hline Mianyang & Sichuan & NEC & 25 & 2 & 8 & 40 & 41 & 3 & 19 & 70 & 21 & 2 & 3 & 49 \\
\hline Nanchong & Sichuan & NEC & 36 & 3 & 15 & 59 & 50 & 3 & 28 & 65 & 38 & 3 & 18 & 77 \\
\hline Panzhihua & Sichuan & NEC & 22 & 1 & 12 & 32 & 28 & 2 & 17 & 40 & 24 & 1 & 9 & 35 \\
\hline Suining & Sichuan & NEC & 29 & 3 & 8 & 51 & 47 & 3 & 18 & 71 & 30 & 3 & 10 & 71 \\
\hline Ya'an & Sichuan & NEC & 15 & 2 & 6 & 36 & 16 & 1 & 10 & 25 & 19 & 2 & 7 & 49 \\
\hline Ziyang & Sichuan & NEC & 18 & 1 & 12 & 31 & 25 & 2 & 15 & 42 & 16 & 1 & 11 & 34 \\
\hline Zigong & Sichuan & NEC & 37 & 4 & 17 & 80 & 57 & 6 & 29 & 88 & 38 & 3 & 15 & 86 \\
\hline Baoshan & Yunnan & NEC & 20 & 1 & 13 & 34 & 18 & 1 & 14 & 23 & 17 & 1 & 11 & 22 \\
\hline Chuxiong & Yunnan & NEC & 14 & 1 & 5 & 26 & 24 & 3 & 6 & 45 & 12 & 1 & 4 & 24 \\
\hline Dali & Yunnan & NEC & 13 & 2 & 4 & 31 & 13 & 1 & 9 & 24 & 11 & 0 & 8 & 15 \\
\hline Dehong & Yunnan & NEC & 21 & 1 & 13 & 33 & 19 & 1 & 12 & 32 & 21 & 1 & 12 & 32 \\
\hline Honghe & Yunnan & NEC & 19 & 4 & 5 & 78 & 34 & 4 & 14 & 68 & 21 & 2 & 10 & 46 \\
\hline Kunming & Yunnan & NEC & 18 & 2 & 9 & 38 & 27 & 3 & 12 & 43 & 20 & 1 & 9 & 37 \\
\hline Lijiang & Yunnan & NEC & 15 & 0 & 14 & 22 & 16 & 0 & 14 & 19 & 17 & 1 & 14 & 31 \\
\hline Lincang & Yunnan & NEC & 15 & 1 & 8 & 26 & 17 & 1 & 14 & 24 & 19 & 1 & 13 & 31 \\
\hline
\end{tabular}




\begin{tabular}{|c|c|c|c|c|c|c|c|c|c|c|c|c|c|c|}
\hline Nujiang & Yunnan & NEC & 12 & 0 & 8 & 15 & 13 & 0 & 11 & 16 & 13 & 0 & 8 & 16 \\
\hline Qujing & Yunnan & NEC & 23 & 2 & 8 & 44 & 34 & 3 & 16 & 51 & 23 & 1 & 6 & 35 \\
\hline Wenshan & Yunnan & NEC & 27 & 2 & 17 & 48 & 40 & 2 & 28 & 53 & 26 & 1 & 16 & 40 \\
\hline Xishuangbanna & Yunnan & NEC & 7 & 1 & 2 & 13 & 16 & 1 & 5 & 23 & 16 & 1 & 6 & 28 \\
\hline Tuxi & Yunnan & NEC & 13 & 1 & 5 & 25 & 26 & 3 & 8 & 40 & 17 & 1 & 7 & 31 \\
\hline Zhaotong & Yunnan & NEC & 31 & 3 & 15 & 56 & 37 & 4 & 10 & 68 & 14 & 1 & 7 & 35 \\
\hline Anshun & Guizhou & NEC & 19 & 1 & 14 & 33 & 34 & 3 & 17 & 57 & 21 & 1 & 14 & 40 \\
\hline Bijie & Guizhou & NEC & 18 & 2 & 6 & 32 & 29 & 3 & 4 & 48 & 16 & 2 & 5 & 38 \\
\hline Guiyang & Guizhou & NEC & 19 & 2 & 7 & 42 & 41 & 3 & 10 & 60 & 25 & 2 & 9 & 55 \\
\hline Liupanshui & Guizhou & NEC & 26 & 3 & 11 & 52 & 42 & 5 & 11 & 67 & 21 & 2 & 8 & 38 \\
\hline Tongren & Guizhou & NEC & 15 & 0 & 13 & 16 & 19 & 1 & 14 & 29 & 22 & 2 & 13 & 47 \\
\hline Zunyi & Guizhou & NEC & 19 & 2 & 7 & 37 & 35 & 3 & 15 & 58 & 28 & 2 & 9 & 58 \\
\hline Baise & Guangxi & NEC & 28 & 3 & 7 & 63 & 37 & 4 & 6 & 59 & 31 & 3 & 11 & 55 \\
\hline Beihai & Guangxi & NEC & 16 & 2 & 6 & 42 & 24 & 5 & 5 & 59 & 22 & 2 & 9 & 53 \\
\hline Chongzuo & Guangxi & NEC & 20 & 2 & 5 & 38 & 29 & 5 & 7 & 63 & 24 & 2 & 8 & 52 \\
\hline Fangchenggang & Guangxi & NEC & 18 & 2 & 7 & 46 & 26 & 6 & 7 & 66 & 23 & 2 & 11 & 50 \\
\hline Guigang & Guangxi & NEC & 37 & 3 & 17 & 73 & 39 & 7 & 11 & 85 & 31 & 2 & 14 & 52 \\
\hline Guilin & Guangxi & NEC & 25 & 2 & 14 & 41 & 43 & 5 & 10 & 66 & 52 & 4 & 19 & 87 \\
\hline Hechi & Guangxi & NEC & 28 & 3 & 16 & 55 & 42 & 5 & 11 & 70 & 35 & 4 & 6 & 86 \\
\hline Hezhou & Guangxi & NEC & 31 & 2 & 20 & 44 & 37 & 6 & 10 & 72 & 31 & 2 & 9 & 46 \\
\hline Laibin & Guangxi & NEC & 34 & 3 & 15 & 70 & 39 & 6 & 11 & 80 & 35 & 3 & 8 & 68 \\
\hline Liuzhou & Guangxi & NEC & 34 & 3 & 16 & 68 & 45 & 7 & 14 & 83 & 42 & 4 & 17 & 77 \\
\hline Nanning & Guangxi & NEC & 27 & 4 & 8 & 62 & 37 & 7 & 7 & 70 & 29 & 3 & 8 & 61 \\
\hline Qinzhou & Guangxi & NEC & 22 & 3 & 10 & 47 & 29 & 6 & 7 & 69 & 26 & 3 & 11 & 55 \\
\hline Wuzhou & Guangxi & NEC & 32 & 3 & 19 & 56 & 36 & 5 & 13 & 60 & 34 & 2 & 14 & 55 \\
\hline Yulin & Guangxi & NEC & 30 & 3 & 11 & 53 & 32 & 5 & 13 & 65 & 29 & 2 & 14 & 50 \\
\hline
\end{tabular}




\begin{tabular}{|c|c|c|c|c|c|c|c|c|c|c|c|c|c|}
\hline Golog & Qinghai & NEC & 34 & 2 & 22 & 47 & 49 & 3 & 40 & 80 & 23 & 3 & 3 \\
\hline Haidong & Qinghai & NEC & 41 & 2 & 28 & 56 & 42 & 4 & 29 & 72 & 35 & 1 & 24 \\
\hline Xining & Qinghai & NEC & 33 & 2 & 15 & 62 & 33 & 2 & 25 & 48 & 32 & 2 & 12 \\
\hline Ali & Tibet & NEC & 14 & 2 & 7 & 33 & 11 & 1 & 5 & 21 & 14 & 1 & 7 \\
\hline Qamdo & Tibet & NEC & 17 & 1 & 9 & 35 & 14 & 1 & 8 & 18 & 15 & 1 & 5 \\
\hline Lhasa & Tibet & NEC & 18 & 1 & 10 & 36 & 19 & 2 & 12 & 39 & 17 & 1 & 11 \\
\hline Nyingchi & Tibet & NEC & 8 & 0 & 5 & 12 & 6 & 0 & 5 & 8 & 6 & 0 & 4 \\
\hline Naqu & Tibet & NEC & 35 & 4 & 16 & 77 & 40 & 4 & 26 & 68 & 47 & 2 & 27 \\
\hline Shigatse & Tibet & NEC & 16 & 2 & 5 & 37 & 13 & 1 & 7 & 26 & 14 & 1 & 5 \\
\hline Lhoka & Tibet & NEC & 9 & 1 & 3 & 14 & 5 & 0 & 4 & 7 & 8 & 0 & 3 \\
\hline
\end{tabular}

118
${ }^{\mathrm{a}} \mathrm{EC}$ and NEC denote emission control and non-emission control regions, respec of emission control measures.

120

b The pre-Parade Blue, Parade Blue, and post-Parade Blue periods indicate the periods of 1-19 August, 20 August-3 September, and 4-30 September 2015, respectively. 
130 Table S3. Summary of daily average $\mathrm{PM}_{10}$ concentrations $\left(\mu \mathrm{g} \mathrm{m}^{-3}\right)$ during the pre-Parade Blue, Parade Blue and post-Parade Blue periods in the 131291 cities across China

\begin{tabular}{|c|c|c|c|c|c|c|c|c|c|c|c|c|c|c|}
\hline \multirow{2}{*}{ City } & \multirow{2}{*}{ Province } & \multirow{2}{*}{ Region $^{a}$} & \multicolumn{4}{|c|}{ Pre-Parade Blue period ${ }^{\mathrm{b}}$} & \multicolumn{4}{|c|}{ Parade Blue period $^{\mathrm{b}}$} & \multicolumn{4}{|c|}{ Post-Parade Blue period ${ }^{\mathrm{b}}$} \\
\hline & & & Mean & $\mathrm{SE}$ & Min & Max & Mean & $\mathrm{SE}$ & Min & $\operatorname{Max}$ & Mean & $\mathrm{SE}$ & Min & Max \\
\hline Beijing & Municipality & EC & 87 & 8 & 44 & 176 & 29 & 3 & 13 & 51 & 65 & 9 & 11 & 163 \\
\hline Tianjing & Municipality & $\mathrm{EC}$ & 111 & 8 & 57 & 200 & 58 & 7 & 23 & 125 & 78 & 7 & 23 & 148 \\
\hline Baoding & Hebei & $\mathrm{EC}$ & 152 & 10 & 61 & 241 & 70 & 8 & 33 & 142 & 115 & 12 & 36 & 250 \\
\hline Cangzhou & Hebei & $\mathrm{EC}$ & 87 & 8 & 44 & 176 & 29 & 3 & 13 & 51 & 70 & 8 & 17 & 152 \\
\hline Chengde & Hebei & EC & 72 & 5 & 43 & 121 & 32 & 2 & 23 & 46 & 58 & 7 & 20 & 154 \\
\hline Handan & Hebei & $\mathrm{EC}$ & 157 & 11 & 84 & 245 & 104 & 14 & 23 & 194 & 124 & 10 & 26 & 224 \\
\hline Hengshui & Hebei & $\mathrm{EC}$ & 121 & 9 & 51 & 221 & 69 & 9 & 12 & 141 & 132 & 13 & 32 & 273 \\
\hline Langfang & Hebei & $\mathrm{EC}$ & 131 & 9 & 47 & 200 & 56 & 8 & 21 & 147 & 106 & 12 & 23 & 229 \\
\hline Qinhuangdao & Hebei & $\mathrm{EC}$ & 90 & 8 & 30 & 175 & 25 & 3 & 13 & 54 & 52 & 6 & 20 & 119 \\
\hline Shijiazhuang & Hebei & $\mathrm{EC}$ & 136 & 11 & 41 & 212 & 70 & 8 & 16 & 127 & 91 & 8 & 29 & 181 \\
\hline Tangshan & Hebei & $\mathrm{EC}$ & 124 & 9 & 52 & 187 & 35 & 4 & 14 & 68 & 87 & 8 & 27 & 163 \\
\hline Xingtai & Hebei & $\mathrm{EC}$ & 127 & 9 & 65 & 208 & 81 & 11 & 20 & 162 & 120 & 10 & 42 & 219 \\
\hline Zhangjiakou & Hebei & $\mathrm{EC}$ & 70 & 14 & 37 & 320 & 40 & 3 & 16 & 54 & 59 & 7 & 22 & 134 \\
\hline Changzhi & Shanxi & $\mathrm{EC}$ & 89 & 7 & 34 & 148 & 63 & 4 & 41 & 86 & 75 & 5 & 32 & 133 \\
\hline Datong & Shanxi & $\mathrm{EC}$ & 70 & 11 & 36 & 250 & 44 & 3 & 24 & 66 & 54 & 5 & 20 & 117 \\
\hline Jincheng & Shanxi & $\mathrm{EC}$ & 92 & 7 & 28 & 139 & 73 & 7 & 39 & 140 & 75 & 6 & 24 & 179 \\
\hline Jinzhong & Shanxi & $\mathrm{EC}$ & 64 & 5 & 32 & 117 & 52 & 4 & 29 & 86 & 66 & 6 & 19 & 126 \\
\hline Linfen & Shanxi & $\mathrm{EC}$ & 58 & 4 & 39 & 118 & 45 & 4 & 25 & 70 & 60 & 4 & 23 & 94 \\
\hline Lvliang & Shanxi & $\mathrm{EC}$ & 66 & 4 & 31 & 101 & 46 & 2 & 34 & 62 & 62 & 4 & 33 & 105 \\
\hline Shuozhou & Shanxi & $\mathrm{EC}$ & 91 & 7 & 47 & 169 & 64 & 4 & 39 & 96 & 60 & 6 & 24 & 128 \\
\hline Taiyuan & Shanxi & $\mathrm{EC}$ & 92 & 7 & 45 & 169 & 58 & 5 & 25 & 97 & 87 & 9 & 23 & 186 \\
\hline
\end{tabular}




\begin{tabular}{|c|c|c|c|c|c|c|c|c|c|c|c|c|c|c|}
\hline Xinzhou & Shanxi & EC & 67 & 4 & 45 & 118 & 42 & 2 & 30 & 51 & 64 & 6 & 24 & 130 \\
\hline Yangquan & Shanxi & EC & 74 & 5 & 36 & 120 & 49 & 4 & 25 & 68 & 69 & 6 & 18 & 128 \\
\hline Yuncheng & Shanxi & EC & 92 & 11 & 38 & 242 & 74 & 6 & 43 & 113 & 55 & 3 & 26 & 102 \\
\hline Binzhou & Shandong & EC & 86 & 7 & 27 & 144 & 59 & 6 & 32 & 118 & 89 & 9 & 25 & 215 \\
\hline Dezhou & Shandong & EC & 137 & 9 & 41 & 204 & 71 & 8 & 25 & 140 & 113 & 11 & 32 & 238 \\
\hline Dongying & Shandong & EC & 109 & 11 & 35 & 196 & 79 & 7 & 44 & 130 & 98 & 9 & 38 & 210 \\
\hline Jinan & Shandong & EC & 101 & 7 & 37 & 137 & 85 & 7 & 50 & 135 & 135 & 9 & 53 & 217 \\
\hline Jining & Shandong & $\mathrm{EC}$ & 80 & 8 & 42 & 154 & 85 & 6 & 52 & 131 & 100 & 6 & 52 & 185 \\
\hline Laiwu & Shandong & $\mathrm{EC}$ & 91 & 9 & 40 & 153 & 96 & 7 & 57 & 156 & 103 & 8 & 41 & 200 \\
\hline Liaocheng & Shandong & EC & 135 & 12 & 47 & 223 & 101 & 11 & 32 & 169 & 132 & 9 & 45 & 216 \\
\hline Linyi & Shandong & EC & 91 & 10 & 36 & 173 & 91 & 8 & 55 & 174 & 101 & 11 & 29 & 262 \\
\hline Qingdao & Shandong & EC & 75 & 5 & 37 & 115 & 68 & 6 & 40 & 135 & 63 & 4 & 35 & 141 \\
\hline Rizhao & Shandong & EC & 69 & 6 & 24 & 123 & 70 & 7 & 29 & 128 & 71 & 7 & 24 & 158 \\
\hline Tai'an & Shandong & EC & 85 & 9 & 39 & 163 & 85 & 7 & 46 & 140 & 103 & 8 & 44 & 196 \\
\hline Weihai & Shandong & EC & 69 & 6 & 39 & 123 & 41 & 3 & 21 & 59 & 41 & 3 & 21 & 73 \\
\hline Weifang & Shandong & EC & 101 & 9 & 47 & 177 & 99 & 12 & 44 & 198 & 100 & 9 & 39 & 253 \\
\hline Yantai & Shandong & EC & 75 & 7 & 36 & 141 & 50 & 4 & 23 & 75 & 53 & 4 & 29 & 120 \\
\hline Zaozhuang & Shandong & EC & 92 & 8 & 46 & 148 & 98 & 5 & 69 & 135 & 117 & 9 & 28 & 245 \\
\hline Zibo & Shandong & EC & 109 & 7 & 56 & 159 & 106 & 9 & 63 & 164 & 129 & 10 & 49 & 241 \\
\hline Alxa League & Inner Mongolia & EC & 96 & 9 & 56 & 195 & 67 & 4 & 33 & 93 & 57 & 7 & 10 & 160 \\
\hline Bayannur & Inner Mongolia & $\mathrm{EC}$ & 82 & 8 & 43 & 193 & 67 & 3 & 54 & 94 & 72 & 5 & 37 & 155 \\
\hline Baotou & Inner Mongolia & EC & 86 & 8 & 35 & 165 & 62 & 3 & 39 & 90 & 71 & 7 & 20 & 145 \\
\hline Chifeng & Inner Mongolia & EC & 59 & 7 & 34 & 156 & 35 & 2 & 24 & 49 & 56 & 5 & 17 & 122 \\
\hline Ordos & Inner Mongolia & EC & 54 & 7 & 20 & 148 & 36 & 2 & 28 & 51 & 46 & 4 & 18 & 97 \\
\hline Hohhot & Inner Mongolia & EC & 87 & 8 & 46 & 207 & 54 & 4 & 33 & 80 & 73 & 8 & 16 & 154 \\
\hline Hulun Buir & Inner Mongolia & EC & 53 & 7 & 16 & 127 & 65 & 5 & 36 & 99 & 54 & 5 & 19 & 115 \\
\hline
\end{tabular}




\begin{tabular}{|c|c|c|c|c|c|c|c|c|c|c|c|c|c|c|}
\hline Tongliao & Inner Mongolia & EC & 95 & 8 & 56 & 165 & 62 & 4 & 46 & 99 & 65 & 6 & 31 & 154 \\
\hline Wuhai & Inner Mongolia & EC & 104 & 12 & 43 & 280 & 67 & 5 & 33 & 104 & 97 & 8 & 38 & 216 \\
\hline Hinggan League & Inner Mongolia & EC & 61 & 3 & 41 & 82 & 43 & 2 & 34 & 53 & 45 & 3 & 21 & 89 \\
\hline Anyang & Henan & EC & 100 & 7 & 49 & 169 & 82 & 9 & 39 & 151 & 118 & 9 & 45 & 256 \\
\hline Hebi & Henan & EC & 99 & 6 & 59 & 144 & 66 & 8 & 27 & 132 & 98 & 8 & 34 & 193 \\
\hline Jiaozuo & Henan & EC & 84 & 6 & 40 & 137 & 71 & 8 & 18 & 123 & 66 & 5 & 33 & 123 \\
\hline Kaifeng & Henan & EC & 76 & 5 & 46 & 131 & 68 & 7 & 35 & 130 & 101 & 7 & 33 & 186 \\
\hline Luoyang & Henan & EC & 96 & 11 & 34 & 223 & 72 & 5 & 31 & 101 & 75 & 5 & 36 & 134 \\
\hline Luohe & Henan & $\mathrm{EC}$ & 80 & 6 & 42 & 117 & 99 & 7 & 57 & 141 & 139 & 8 & 73 & 271 \\
\hline Nanyang & Henan & EC & 93 & 6 & 48 & 140 & 109 & 7 & 67 & 148 & 104 & 7 & 57 & 176 \\
\hline Pingdingshan & Henan & EC & 91 & 8 & 42 & 148 & 91 & 6 & 61 & 133 & 114 & 8 & 52 & 217 \\
\hline Sanmenxia & Henan & EC & 100 & 12 & 29 & 255 & 72 & 5 & 42 & 112 & 71 & 5 & 24 & 112 \\
\hline Shangqiu & Henan & EC & 65 & 6 & 30 & 141 & 65 & 6 & 31 & 112 & 83 & 7 & 40 & 158 \\
\hline Xinyang & Henan & EC & 56 & 5 & 30 & 104 & 78 & 4 & 45 & 103 & 86 & 6 & 30 & 135 \\
\hline Zhengzhou & Henan & EC & 107 & 9 & 44 & 187 & 97 & 12 & 37 & 193 & 135 & 10 & 50 & 255 \\
\hline Zhoukou & Henan & EC & 57 & 4 & 29 & 92 & 68 & 5 & 36 & 94 & 103 & 7 & 48 & 193 \\
\hline Zhumadian & Henan & $\mathrm{EC}$ & 78 & 6 & 47 & 125 & 88 & 8 & 32 & 128 & 100 & 8 & 32 & 196 \\
\hline Anshan & Liaoning & NEC & 61 & 5 & 24 & 101 & 70 & 4 & 52 & 101 & 76 & 5 & 38 & 139 \\
\hline Benxi & Liaoning & NEC & 57 & 5 & 29 & 108 & 57 & 3 & 29 & 80 & 67 & 4 & 27 & 112 \\
\hline Chaoyang & Liaoning & NEC & 61 & 6 & 34 & 139 & 41 & 2 & 30 & 56 & 64 & 5 & 24 & 146 \\
\hline Dalian & Liaoning & NEC & 67 & 8 & 25 & 141 & 46 & 4 & 22 & 81 & 49 & 4 & 22 & 97 \\
\hline Dandong & Liaoning & NEC & 28 & 7 & 0 & 90 & 36 & 2 & 19 & 49 & 50 & 3 & 19 & 84 \\
\hline Fushun & Liaoning & NEC & 47 & 4 & 25 & 90 & 36 & 3 & 19 & 53 & 60 & 5 & 19 & 98 \\
\hline Fuxin & Liaoning & NEC & 72 & 8 & 27 & 143 & 30 & 2 & 17 & 49 & 54 & 4 & 22 & 117 \\
\hline Huludao & Liaoning & NEC & 94 & 12 & 31 & 191 & 45 & 3 & 33 & 75 & 62 & 5 & 22 & 128 \\
\hline Jinzhou & Liaoning & NEC & 83 & 10 & 38 & 174 & 37 & 3 & 29 & 67 & 60 & 4 & 28 & 114 \\
\hline
\end{tabular}




\begin{tabular}{|c|c|c|c|c|c|c|c|c|c|c|c|c|c|c|}
\hline Liaoyang & Liaoning & NEC & 60 & 5 & 21 & 103 & 64 & 3 & 44 & 82 & 78 & 6 & 32 & 140 \\
\hline Panjin & Liaoning & NEC & 63 & 8 & 23 & 141 & 33 & 3 & 25 & 69 & 44 & 3 & 22 & 78 \\
\hline Shenyang & Liaoning & NEC & 62 & 5 & 30 & 99 & 46 & 3 & 30 & 69 & 69 & 5 & 27 & 130 \\
\hline Tieling & Liaoning & NEC & 48 & 4 & 26 & 83 & 34 & 2 & 15 & 45 & 66 & 5 & 28 & 119 \\
\hline Wafangdian & Liaoning & NEC & 57 & 6 & 21 & 93 & 45 & 3 & 25 & 69 & 55 & 4 & 18 & 92 \\
\hline Yingkou & Liaoning & NEC & 54 & 5 & 23 & 109 & 44 & 7 & 14 & 122 & 39 & 5 & 8 & 106 \\
\hline Baicheng & Jilin & NEC & 63 & 3 & 42 & 83 & 51 & 2 & 39 & 61 & 57 & 3 & 33 & 103 \\
\hline Baishan & Jilin & NEC & 50 & 3 & 26 & 82 & 39 & 2 & 22 & 52 & 59 & 3 & 36 & 92 \\
\hline Changchun & Jilin & NEC & 52 & 4 & 26 & 103 & 42 & 4 & 15 & 64 & 64 & 4 & 28 & 118 \\
\hline Jilin & Jilin & NEC & 38 & 3 & 23 & 59 & 38 & 3 & 14 & 54 & 55 & 2 & 31 & 89 \\
\hline Liaoyuan & Jilin & NEC & 46 & 6 & 21 & 91 & 48 & 8 & 18 & 103 & 53 & 3 & 30 & 96 \\
\hline Siping & Jilin & NEC & 59 & 5 & 27 & 118 & 40 & 3 & 18 & 54 & 63 & 4 & 26 & 119 \\
\hline Songyuan & Jilin & NEC & 34 & 2 & 20 & 55 & 23 & 2 & 8 & 37 & 40 & 3 & 14 & 71 \\
\hline Daqing & Heilongjiang & NEC & 47 & 2 & 33 & 75 & 38 & 2 & 26 & 48 & 40 & 3 & 22 & 74 \\
\hline Daxinganling & Heilongjiang & NEC & 48 & 3 & 31 & 88 & 43 & 2 & 35 & 53 & 44 & 3 & 25 & 84 \\
\hline Harbin & Heilongjiang & NEC & 45 & 2 & 26 & 59 & 32 & 2 & 15 & 47 & 51 & 3 & 26 & 92 \\
\hline Hegang & Heilongjiang & NEC & 80 & 4 & 48 & 123 & 48 & 5 & 21 & 100 & 26 & 3 & 7 & 57 \\
\hline Heihe & Heilongjiang & NEC & 56 & 3 & 32 & 81 & 41 & 2 & 30 & 56 & 24 & 3 & 7 & 56 \\
\hline Jixi & Heilongjiang & NEC & 35 & 2 & 24 & 46 & 30 & 3 & 15 & 54 & 50 & 3 & 21 & 87 \\
\hline Jiamusi & Heilongjiang & NEC & 29 & 2 & 16 & 43 & 26 & 2 & 14 & 44 & 19 & 1 & 7 & 32 \\
\hline Mudanjiang & Heilongjiang & NEC & 38 & 1 & 27 & 53 & 45 & 2 & 31 & 59 & 55 & 3 & 34 & 92 \\
\hline Qitaihe & Heilongjiang & NEC & 36 & 2 & 21 & 56 & 32 & 3 & 14 & 51 & 52 & 3 & 32 & 99 \\
\hline Qiqihar & Heilongjiang & NEC & 47 & 3 & 30 & 68 & 42 & 2 & 31 & 56 & 45 & 3 & 24 & 91 \\
\hline Shuangyashan & Heilongjiang & NEC & 44 & 2 & 35 & 57 & 45 & 3 & 27 & 63 & 39 & 2 & 24 & 64 \\
\hline Suihua & Heilongjiang & NEC & 23 & 2 & 14 & 40 & 21 & 1 & 15 & 32 & 30 & 2 & 13 & 57 \\
\hline Ankang & Shaanxi & NEC & 34 & 3 & 11 & 60 & 43 & 2 & 26 & 59 & 53 & 3 & 20 & 92 \\
\hline
\end{tabular}




\begin{tabular}{|c|c|c|c|c|c|c|c|c|c|c|c|c|c|c|}
\hline Baoji & Shaanxi & NEC & 87 & 14 & 31 & 286 & 73 & 5 & 42 & 113 & 69 & 5 & 18 & 122 \\
\hline Hanzhong & Shaanxi & NEC & 42 & 5 & 19 & 126 & 50 & 2 & 33 & 58 & 45 & 3 & 12 & 75 \\
\hline Shangluo & Shaanxi & NEC & 53 & 10 & 18 & 213 & 45 & 3 & 28 & 57 & 53 & 4 & 25 & 88 \\
\hline Tongchuan & Shaanxi & NEC & 75 & 9 & 35 & 178 & 64 & 2 & 46 & 82 & 71 & 5 & 26 & 107 \\
\hline Weinan & Shaanxi & NEC & 79 & 11 & 16 & 219 & 74 & 4 & 46 & 102 & 94 & 7 & 37 & 178 \\
\hline Xi'an & Shaanxi & NEC & 106 & 15 & 30 & 285 & 94 & 5 & 61 & 136 & 80 & 5 & 24 & 123 \\
\hline Xianyang & Shaanxi & NEC & 79 & 10 & 22 & 189 & 78 & 4 & 43 & 104 & 78 & 5 & 26 & 129 \\
\hline Yan'an & Shaanxi & NEC & 76 & 8 & 39 & 194 & 67 & 3 & 53 & 95 & 73 & 5 & 21 & 119 \\
\hline Yulin & Shaanxi & NEC & 70 & 8 & 26 & 160 & 59 & 5 & 39 & 97 & 63 & 5 & 19 & 133 \\
\hline Baiying & Gansu & NEC & 71 & 11 & 19 & 247 & 71 & 4 & 37 & 100 & 73 & 8 & 18 & 192 \\
\hline Dingxi & Gansu & NEC & 49 & 4 & 19 & 74 & 59 & 4 & 39 & 91 & 42 & 2 & 23 & 67 \\
\hline Gannan & Gansu & NEC & 42 & 4 & 19 & 80 & 68 & 7 & 43 & 140 & 38 & 3 & 15 & 63 \\
\hline Jiayuguan & Gansu & NEC & 85 & 22 & 31 & 421 & 82 & 11 & 38 & 212 & 87 & 21 & 15 & 535 \\
\hline Jiuquan & Gansu & NEC & 126 & 39 & 45 & 662 & 96 & 8 & 49 & 180 & 82 & 15 & 27 & 458 \\
\hline Lanzhou & Gansu & NEC & 90 & 10 & 36 & 237 & 81 & 6 & 45 & 117 & 80 & 6 & 24 & 163 \\
\hline Linxia & Gansu & NEC & 46 & 3 & 20 & 82 & 44 & 2 & 34 & 57 & 40 & 2 & 26 & 57 \\
\hline Pingliang & Gansu & NEC & 59 & 6 & 22 & 135 & 41 & 2 & 27 & 51 & 46 & 4 & 19 & 102 \\
\hline Qingyang & Gansu & NEC & 54 & 10 & 14 & 186 & 40 & 2 & 27 & 54 & 36 & 3 & 12 & 63 \\
\hline Tianshui & Gansu & NEC & 43 & 9 & 14 & 200 & 28 & 2 & 16 & 41 & 22 & 1 & 9 & 43 \\
\hline Wuwei & Gansu & NEC & 80 & 13 & 39 & 284 & 74 & 7 & 34 & 129 & 51 & 4 & 15 & 115 \\
\hline Zhangye & Gansu & NEC & 50 & 11 & 20 & 199 & 34 & 4 & 9 & 58 & 40 & 7 & 17 & 212 \\
\hline Guyuan & Ningxia & NEC & 75 & 11 & 27 & 232 & 55 & 3 & 28 & 71 & 54 & 6 & 14 & 157 \\
\hline Shizuishan & Ningxia & NEC & 86 & 12 & 40 & 258 & 64 & 5 & 39 & 90 & 81 & 9 & 31 & 252 \\
\hline Wuzhong & Ningxia & NEC & 75 & 9 & 35 & 157 & 78 & 4 & 51 & 99 & 68 & 5 & 26 & 156 \\
\hline Yinchuan & Ningxia & NEC & 96 & 8 & 58 & 187 & 74 & 5 & 44 & 104 & 69 & 4 & 30 & 107 \\
\hline Zhongwei & Ningxia & NEC & 65 & 8 & 30 & 168 & 63 & 4 & 29 & 79 & 58 & 7 & 12 & 201 \\
\hline
\end{tabular}




\begin{tabular}{|c|c|c|c|c|c|c|c|c|c|c|c|c|c|c|}
\hline Aksu & Sinkiang & NEC & 255 & 46 & 46 & 804 & 118 & 18 & 48 & 231 & 118 & 17 & 31 & 466 \\
\hline Hami & Sinkiang & NEC & 79 & 8 & 39 & 197 & 114 & 16 & 72 & 317 & 81 & 7 & 34 & 176 \\
\hline Hotan & Sinkiang & NEC & 485 & 117 & 77 & 2562 & 296 & 42 & 67 & 628 & 176 & 25 & 52 & 627 \\
\hline Kashgar & Sinkiang & NEC & 295 & 63 & 97 & 1039 & 151 & 32 & 43 & 413 & 194 & 25 & 57 & 534 \\
\hline Karamay & Sinkiang & NEC & 53 & 5 & 21 & 139 & 58 & 8 & 31 & 147 & 43 & 4 & 23 & 134 \\
\hline Kizilsu & Sinkiang & NEC & 213 & 46 & 21 & 757 & 97 & 24 & 23 & 291 & 135 & 21 & 25 & 438 \\
\hline Shihezi & Sinkiang & NEC & 79 & 19 & 18 & 369 & 59 & 8 & 24 & 134 & 55 & 6 & 11 & 139 \\
\hline Urumchi & Sinkiang & NEC & 95 & 9 & 30 & 209 & 102 & 15 & 38 & 208 & 81 & 8 & 18 & 183 \\
\hline Wujiaqu & Sinkiang & NEC & 86 & 11 & 26 & 225 & 95 & 18 & 21 & 229 & 68 & 9 & 9 & 200 \\
\hline Yili & Sinkiang & NEC & 64 & 4 & 27 & 97 & 66 & 9 & 17 & 127 & 62 & 7 & 16 & 167 \\
\hline Ezhou & Hubei & NEC & 61 & 5 & 16 & 98 & 79 & 5 & 36 & 110 & 78 & 3 & 32 & 106 \\
\hline Huanggang & Hubei & NEC & 48 & 4 & 18 & 87 & 63 & 3 & 29 & 83 & 64 & 3 & 32 & 98 \\
\hline Jingmen & Hubei & NEC & 58 & 6 & 20 & 135 & 90 & 6 & 44 & 122 & 98 & 6 & 41 & 165 \\
\hline Shiyan & Hubei & NEC & 48 & 4 & 21 & 85 & 63 & 5 & 38 & 92 & 77 & 6 & 25 & 148 \\
\hline Wuhan & Hubei & NEC & 66 & 6 & 13 & 116 & 103 & 8 & 42 & 158 & 85 & 5 & 27 & 143 \\
\hline Xianning & Hubei & NEC & 55 & 4 & 28 & 93 & 83 & 4 & 41 & 96 & 67 & 3 & 34 & 87 \\
\hline Xiangyang & Hubei & NEC & 59 & 5 & 26 & 112 & 80 & 5 & 51 & 115 & 91 & 6 & 32 & 142 \\
\hline Xiaogan & Hubei & NEC & 55 & 4 & 30 & 84 & 79 & 5 & 45 & 108 & 81 & 4 & 47 & 138 \\
\hline Yichang & Hubei & NEC & 58 & 6 & 21 & 117 & 85 & 5 & 55 & 131 & 77 & 5 & 24 & 131 \\
\hline Anqing & Anhui & NEC & 58 & 4 & 25 & 80 & 53 & 4 & 24 & 77 & 49 & 2 & 21 & 70 \\
\hline Bengbu & Anhui & NEC & 64 & 5 & 19 & 102 & 85 & 6 & 32 & 136 & 77 & 5 & 23 & 141 \\
\hline Bozhou & Anhui & NEC & 60 & 4 & 37 & 98 & 85 & 6 & 48 & 114 & 92 & 5 & 29 & 164 \\
\hline Chuzhou & Anhui & NEC & 69 & 6 & 20 & 112 & 81 & 7 & 34 & 125 & 77 & 5 & 50 & 145 \\
\hline Fuyang & Anhui & NEC & 59 & 4 & 28 & 94 & 77 & 5 & 45 & 133 & 61 & 3 & 21 & 98 \\
\hline Hefei & Anhui & NEC & 67 & 5 & 14 & 99 & 79 & 6 & 26 & 120 & 69 & 4 & 25 & 102 \\
\hline Huaibei & Anhui & NEC & 66 & 7 & 27 & 129 & 63 & 4 & 42 & 84 & 85 & 7 & 23 & 168 \\
\hline
\end{tabular}




\begin{tabular}{|c|c|c|c|c|c|c|c|c|c|c|c|c|c|c|}
\hline Huainan & Anhui & NEC & 53 & 4 & 32 & 85 & 78 & 6 & 35 & 118 & 75 & 4 & 35 & 130 \\
\hline Huangshan & Anhui & NEC & 30 & 2 & 14 & 43 & 46 & 5 & 19 & 83 & 40 & 2 & 21 & 62 \\
\hline Lu'an & Anhui & NEC & 58 & 4 & 20 & 96 & 79 & 4 & 48 & 103 & 70 & 3 & 23 & 98 \\
\hline Ma'anshan & Anhui & NEC & 60 & 4 & 20 & 84 & 79 & 7 & 34 & 120 & 64 & 3 & 38 & 115 \\
\hline Suzhou & Anhui & NEC & 72 & 8 & 29 & 142 & 85 & 6 & 45 & 119 & 87 & 7 & 26 & 203 \\
\hline Tongling & Anhui & NEC & 83 & 6 & 38 & 142 & 102 & 9 & 49 & 150 & 69 & 3 & 39 & 96 \\
\hline Wuhu & Anhui & NEC & 54 & 3 & 26 & 78 & 74 & 5 & 35 & 107 & 68 & 4 & 40 & 107 \\
\hline Changzhou & Jiangsu & NEC & 66 & 7 & 24 & 126 & 79 & 9 & 14 & 121 & 67 & 5 & 37 & 127 \\
\hline Huaian & Jiangsu & NEC & 57 & 5 & 18 & 100 & 72 & 6 & 20 & 120 & 56 & 4 & 17 & 103 \\
\hline Lianyungang & Jiangsu & NEC & 55 & 5 & 23 & 92 & 69 & 7 & 24 & 139 & 56 & 5 & 17 & 121 \\
\hline Nanjing & Jiangsu & NEC & 61 & 5 & 12 & 89 & 69 & 7 & 27 & 105 & 60 & 4 & 30 & 112 \\
\hline Nantong & Jiangsu & NEC & 56 & 5 & 21 & 91 & 59 & 8 & 15 & 136 & 51 & 4 & 27 & 99 \\
\hline Suzhou & Jiangsu & NEC & 60 & 5 & 26 & 92 & 76 & 8 & 23 & 125 & 57 & 4 & 23 & 107 \\
\hline Suqian & Jiangsu & NEC & 56 & 5 & 24 & 94 & 67 & 4 & 28 & 104 & 64 & 6 & 17 & 165 \\
\hline Taizhou & Jiangsu & NEC & 74 & 7 & 24 & 128 & 75 & 7 & 28 & 123 & 67 & 3 & 43 & 109 \\
\hline Wuxi & Jiangsu & NEC & 61 & 5 & 27 & 96 & 79 & 8 & 25 & 130 & 63 & 4 & 30 & 114 \\
\hline Xuzhou & Jiangsu & NEC & 62 & 5 & 25 & 109 & 78 & 4 & 53 & 104 & 77 & 6 & 27 & 155 \\
\hline Yanchen & Jiangsu & NEC & 56 & 6 & 19 & 104 & 51 & 4 & 16 & 81 & 48 & 3 & 27 & 87 \\
\hline Yangzhou & Jiangsu & NEC & 64 & 6 & 20 & 112 & 63 & 5 & 30 & 82 & 57 & 4 & 31 & 106 \\
\hline Zhenjiang & Jiangsu & NEC & 50 & 5 & 12 & 92 & 57 & 6 & 14 & 86 & 50 & 4 & 24 & 112 \\
\hline Shanghai & Municipality & NEC & 52 & 4 & 27 & 92 & 72 & 10 & 17 & 149 & 43 & 3 & 23 & 84 \\
\hline Hangzhou & Zhejiang & NEC & 53 & 5 & 24 & 96 & 64 & 7 & 20 & 107 & 61 & 4 & 18 & 106 \\
\hline Huzhou & Zhejiang & NEC & 48 & 4 & 21 & 80 & 64 & 7 & 19 & 99 & 50 & 3 & 25 & 109 \\
\hline Jiaxing & Zhejiang & NEC & 51 & 4 & 29 & 74 & 72 & 8 & 25 & 134 & 55 & 3 & 24 & 117 \\
\hline Jinhua & Zhejiang & NEC & 49 & 3 & 18 & 78 & 69 & 8 & 18 & 118 & 61 & 4 & 22 & 114 \\
\hline Lishui & Zhejiang & NEC & 39 & 3 & 12 & 59 & 47 & 3 & 26 & 66 & 49 & 4 & 12 & 97 \\
\hline
\end{tabular}




\begin{tabular}{|c|c|c|c|c|c|c|c|c|c|c|c|c|c|c|}
\hline Ningbo & Zhejiang & NEC & 38 & 2 & 24 & 65 & 51 & 6 & 17 & 101 & 49 & 3 & 20 & 83 \\
\hline Quzhou & Zhejiang & NEC & 49 & 3 & 18 & 78 & 70 & 8 & 18 & 110 & 54 & 4 & 23 & 96 \\
\hline Shaoxing & Zhejiang & NEC & 48 & 4 & 23 & 89 & 76 & 9 & 24 & 138 & 63 & 4 & 18 & 100 \\
\hline Taizhou & Zhejiang & NEC & 41 & 2 & 31 & 57 & 45 & 5 & 20 & 81 & 48 & 2 & 30 & 85 \\
\hline Wenjiang & Zhejiang & NEC & 48 & 3 & 29 & 73 & 48 & 4 & 24 & 71 & 51 & 2 & 29 & 78 \\
\hline Zhoushan & Zhejiang & NEC & 28 & 1 & 22 & 44 & 34 & 5 & 11 & 72 & 31 & 2 & 17 & 65 \\
\hline Fuzhou & Jiangxi & NEC & 46 & 4 & 26 & 79 & 67 & 6 & 30 & 106 & 61 & 4 & 21 & 99 \\
\hline Ganzhou & Jiangxi & NEC & 60 & 5 & 33 & 102 & 61 & 5 & 32 & 99 & 61 & 3 & 33 & 93 \\
\hline Ji'an & Jiangxi & NEC & 51 & 4 & 23 & 89 & 57 & 6 & 24 & 100 & 55 & 3 & 23 & 75 \\
\hline Jingdezhen & Jiangxi & NEC & 53 & 5 & 20 & 85 & 71 & 6 & 31 & 99 & 51 & 3 & 19 & 81 \\
\hline Jiujiang & Jiangxi & NEC & 53 & 4 & 17 & 81 & 74 & 6 & 38 & 103 & 55 & 3 & 13 & 80 \\
\hline Nanchang & Jiangxi & NEC & 66 & 7 & 19 & 118 & 80 & 8 & 31 & 149 & 57 & 3 & 12 & 88 \\
\hline Pingxiang & Jiangxi & NEC & 47 & 3 & 29 & 79 & 83 & 7 & 33 & 124 & 73 & 4 & 30 & 104 \\
\hline Shangrao & Jiangxi & NEC & 54 & 4 & 23 & 77 & 62 & 6 & 30 & 108 & 55 & 3 & 30 & 95 \\
\hline Xinyu & Jiangxi & NEC & 53 & 3 & 26 & 79 & 70 & 5 & 35 & 99 & 63 & 2 & 32 & 81 \\
\hline Yichun & Jiangxi & NEC & 44 & 2 & 33 & 70 & 68 & 4 & 43 & 93 & 65 & 3 & 34 & 85 \\
\hline Yingtan & Jiangxi & NEC & 39 & 4 & 13 & 64 & 61 & 5 & 35 & 88 & 57 & 3 & 21 & 85 \\
\hline Changsha & Hunan & NEC & 47 & 3 & 15 & 72 & 85 & 6 & 31 & 124 & 72 & 5 & 25 & 124 \\
\hline Changde & Hunan & NEC & 41 & 3 & 17 & 62 & 59 & 4 & 25 & 80 & 65 & 4 & 28 & 104 \\
\hline Chenzhou & Hunan & NEC & 53 & 4 & 30 & 81 & 71 & 9 & 28 & 157 & 80 & 5 & 39 & 140 \\
\hline Huaihua & Hunan & NEC & 49 & 3 & 33 & 71 & 91 & 6 & 36 & 125 & 84 & 6 & 30 & 149 \\
\hline Loudi & Hunan & NEC & 52 & 3 & 28 & 88 & 87 & 6 & 41 & 117 & 73 & 5 & 26 & 139 \\
\hline Xiangtan & Hunan & NEC & 48 & 3 & 25 & 76 & 90 & 6 & 37 & 123 & 79 & 5 & 32 & 150 \\
\hline Yiyang & Hunan & NEC & 48 & 3 & 29 & 70 & 79 & 4 & 40 & 104 & 80 & 5 & 38 & 168 \\
\hline Yongzhou & Hunan & NEC & 57 & 3 & 40 & 88 & 69 & 5 & 37 & 100 & 81 & 6 & 39 & 183 \\
\hline Zhangjiajie & Hunan & NEC & 44 & 3 & 18 & 80 & 71 & 5 & 35 & 102 & 71 & 5 & 15 & 109 \\
\hline
\end{tabular}




\begin{tabular}{|c|c|c|c|c|c|c|c|c|c|c|c|c|c|c|}
\hline Zhuzhou & Hunan & NEC & 42 & 3 & 23 & 70 & 81 & 7 & 38 & 134 & 69 & 4 & 28 & 104 \\
\hline Dongguan & Guangdong & NEC & 39 & 2 & 21 & 63 & 50 & 4 & 27 & 78 & 55 & 3 & 31 & 93 \\
\hline Foshan & Guangdong & NEC & 52 & 3 & 35 & 83 & 63 & 6 & 27 & 105 & 55 & 3 & 27 & 81 \\
\hline Guangzhou & Guangdong & NEC & 55 & 2 & 34 & 75 & 57 & 5 & 31 & 93 & 65 & 3 & 37 & 100 \\
\hline Heyuan & Guangdong & NEC & 37 & 2 & 20 & 55 & 45 & 5 & 22 & 79 & 55 & 3 & 24 & 89 \\
\hline Huizhou & Guangdong & NEC & 44 & 3 & 24 & 72 & 45 & 5 & 19 & 76 & 43 & 4 & 20 & 82 \\
\hline Jiangmen & Guangdong & NEC & 33 & 3 & 16 & 58 & 52 & 7 & 18 & 94 & 49 & 3 & 27 & 80 \\
\hline Maoming & Guangdong & NEC & 34 & 4 & 13 & 81 & 46 & 8 & 9 & 94 & 43 & 4 & 20 & 83 \\
\hline Meizhou & Guangdong & NEC & 38 & 3 & 18 & 61 & 39 & 5 & 16 & 71 & 50 & 3 & 24 & 86 \\
\hline Qingyuan & Guangdong & NEC & 51 & 3 & 29 & 80 & 44 & 6 & 19 & 84 & 49 & 4 & 16 & 115 \\
\hline Shantou & Guangdong & NEC & 42 & 3 & 20 & 66 & 42 & 5 & 25 & 74 & 48 & 2 & 30 & 77 \\
\hline Shaoguan & Guangdong & NEC & 47 & 3 & 29 & 82 & 46 & 6 & 20 & 79 & 59 & 4 & 17 & 108 \\
\hline Shenzhen & Guangdong & NEC & 37 & 4 & 20 & 78 & 44 & 6 & 20 & 77 & 47 & 3 & 28 & 79 \\
\hline Zhujiang & Guangdong & NEC & 33 & 3 & 13 & 64 & 50 & 8 & 16 & 99 & 40 & 3 & 21 & 85 \\
\hline Zhaoqing & Guangdong & NEC & 47 & 3 & 27 & 77 & 54 & 6 & 27 & 104 & 55 & 4 & 17 & 96 \\
\hline Zhongshan & Guangdong & NEC & 31 & 4 & 15 & 76 & 39 & 6 & 11 & 80 & 43 & 3 & 21 & 81 \\
\hline Zhuhai & Guangdong & NEC & 38 & 5 & 21 & 95 & 47 & 8 & 17 & 101 & 53 & 4 & 21 & 108 \\
\hline Fuzhou & Fujian & NEC & 44 & 4 & 11 & 86 & 38 & 3 & 19 & 55 & 42 & 2 & 17 & 65 \\
\hline Longyan & Fujian & NEC & 33 & 3 & 10 & 65 & 34 & 4 & 17 & 58 & 46 & 3 & 19 & 78 \\
\hline Nanping & Fujian & NEC & 25 & 2 & 7 & 48 & 26 & 3 & 13 & 51 & 31 & 2 & 16 & 51 \\
\hline Ningde & Fujian & NEC & 35 & 3 & 10 & 59 & 31 & 2 & 20 & 43 & 34 & 2 & 14 & 51 \\
\hline Putian & Fujian & NEC & 38 & 3 & 18 & 58 & 31 & 2 & 19 & 41 & 40 & 2 & 21 & 56 \\
\hline Quanzhou & Fujian & NEC & 45 & 5 & 10 & 98 & 30 & 4 & 18 & 59 & 46 & 3 & 22 & 92 \\
\hline Sanming & Fujian & NEC & 44 & 4 & 11 & 74 & 41 & 5 & 18 & 78 & 48 & 4 & 11 & 81 \\
\hline Xiamen & Fujian & NEC & 37 & 3 & 21 & 65 & 32 & 2 & 21 & 51 & 45 & 2 & 20 & 62 \\
\hline Zhangzhou & Fujian & NEC & 43 & 3 & 24 & 80 & 42 & 2 & 33 & 59 & 52 & 2 & 24 & 84 \\
\hline
\end{tabular}




\begin{tabular}{|c|c|c|c|c|c|c|c|c|c|c|c|c|c|c|}
\hline Haikou & Hainan & NEC & 28 & 2 & 19 & 46 & 34 & 4 & 20 & 68 & 30 & 2 & 12 & 65 \\
\hline Sanya & Hainan & NEC & 21 & 1 & 16 & 30 & 24 & 2 & 15 & 33 & 23 & 1 & 10 & 35 \\
\hline Chongqing & Municipality & NEC & 58 & 5 & 21 & 104 & 82 & 5 & 42 & 106 & 49 & 4 & 19 & 86 \\
\hline Bazhong & Sichuan & NEC & 27 & 2 & 11 & 41 & 35 & 2 & 25 & 46 & 30 & 2 & 12 & 54 \\
\hline Chengdu & Sichuan & NEC & 62 & 7 & 15 & 134 & 88 & 8 & 42 & 139 & 53 & 5 & 16 & 158 \\
\hline Dazhou & Sichuan & NEC & 49 & 3 & 12 & 73 & 86 & 5 & 53 & 121 & 62 & 4 & 26 & 96 \\
\hline Deyang & Sichuan & NEC & 56 & 4 & 22 & 87 & 89 & 9 & 43 & 169 & 49 & 4 & 19 & 96 \\
\hline Guangyuan & Sichuan & NEC & 35 & 4 & 16 & 101 & 38 & 2 & 29 & 50 & 31 & 2 & 15 & 50 \\
\hline Leshan & Sichuan & NEC & 44 & 6 & 14 & 126 & 78 & 9 & 27 & 150 & 47 & 4 & 23 & 126 \\
\hline Luzhou & Sichuan & NEC & 49 & 7 & 14 & 113 & 80 & 9 & 32 & 145 & 43 & 3 & 14 & 71 \\
\hline Meishan & Sichuan & NEC & 54 & 5 & 14 & 84 & 79 & 8 & 38 & 136 & 45 & 5 & 15 & 159 \\
\hline Mianyang & Sichuan & NEC & 40 & 3 & 12 & 62 & 63 & 5 & 26 & 98 & 35 & 3 & 7 & 83 \\
\hline Nanchong & Sichuan & NEC & 54 & 5 & 20 & 93 & 71 & 4 & 41 & 97 & 51 & 4 & 25 & 113 \\
\hline Panzhihua & Sichuan & NEC & 44 & 3 & 22 & 69 & 53 & 4 & 34 & 81 & 53 & 2 & 22 & 71 \\
\hline Suining & Sichuan & NEC & 53 & 5 & 21 & 92 & 72 & 4 & 39 & 105 & 50 & 4 & 25 & 110 \\
\hline Ya'an & Sichuan & NEC & 30 & 3 & 12 & 57 & 35 & 2 & 24 & 58 & 29 & 2 & 14 & 69 \\
\hline Ziyang & Sichuan & NEC & 41 & 3 & 24 & 62 & 60 & 5 & 38 & 91 & 37 & 2 & 23 & 87 \\
\hline Zigong & Sichuan & NEC & 59 & 6 & 30 & 115 & 85 & 8 & 44 & 128 & 60 & 4 & 33 & 120 \\
\hline Baoshan & Yunnan & NEC & 37 & 2 & 27 & 51 & 37 & 1 & 29 & 46 & 32 & 2 & 7 & 50 \\
\hline Chuxiong & Yunnan & NEC & 26 & 2 & 14 & 41 & 41 & 6 & 11 & 75 & 23 & 2 & 11 & 38 \\
\hline Dali & Yunnan & NEC & 15 & 1 & 4 & 27 & 18 & 2 & 8 & 37 & 17 & 1 & 10 & 25 \\
\hline Dehong & Yunnan & NEC & 34 & 2 & 19 & 51 & 30 & 2 & 23 & 46 & 37 & 2 & 20 & 55 \\
\hline Honghe & Yunnan & NEC & 38 & 5 & 17 & 108 & 59 & 7 & 26 & 99 & 32 & 2 & 16 & 59 \\
\hline Kunming & Yunnan & NEC & 34 & 3 & 17 & 65 & 48 & 5 & 22 & 76 & 41 & 2 & 23 & 64 \\
\hline Lijiang & Yunnan & NEC & 30 & 1 & 24 & 37 & 33 & 1 & 29 & 43 & 35 & 1 & 28 & 41 \\
\hline Lincang & Yunnan & NEC & 27 & 1 & 19 & 40 & 27 & 1 & 22 & 38 & 28 & 1 & 23 & 60 \\
\hline
\end{tabular}




\begin{tabular}{|c|c|c|c|c|c|c|c|c|c|c|c|c|c|c|}
\hline Nujiang & Yunnan & NEC & 24 & 1 & 16 & 35 & 27 & 1 & 21 & 38 & 26 & 1 & 20 & 34 \\
\hline Qujing & Yunnan & NEC & 30 & 2 & 14 & 49 & 44 & 4 & 21 & 67 & 33 & 2 & 15 & 47 \\
\hline Wenshan & Yunnan & NEC & 26 & 3 & 7 & 68 & 45 & 3 & 24 & 64 & 26 & 2 & 12 & 40 \\
\hline Xishuangbanna & Yunnan & NEC & 22 & 1 & 10 & 31 & 29 & 2 & 15 & 40 & 40 & 2 & 20 & 57 \\
\hline Tuxi & Yunnan & NEC & 25 & 2 & 9 & 40 & 45 & 4 & 16 & 69 & 30 & 2 & 14 & 57 \\
\hline Zhaotong & Yunnan & NEC & 47 & 4 & 23 & 88 & 59 & 6 & 21 & 92 & 33 & 2 & 16 & 57 \\
\hline Anshun & Guizhou & NEC & 36 & 3 & 24 & 63 & 69 & 8 & 28 & 124 & 38 & 3 & 24 & 76 \\
\hline Bijie & Guizhou & NEC & 28 & 3 & 8 & 47 & 43 & 4 & 6 & 74 & 24 & 2 & 9 & 52 \\
\hline Guiyang & Guizhou & NEC & 34 & 4 & 16 & 79 & 63 & 6 & 13 & 97 & 39 & 3 & 17 & 78 \\
\hline Liupanshui & Guizhou & NEC & 51 & 5 & 24 & 94 & 74 & 7 & 25 & 120 & 39 & 2 & 15 & 59 \\
\hline Tongren & Guizhou & NEC & 27 & 1 & 25 & 35 & 41 & 2 & 28 & 54 & 40 & 3 & 23 & 75 \\
\hline Zunyi & Guizhou & NEC & 50 & 3 & 28 & 74 & 74 & 6 & 35 & 113 & 55 & 4 & 29 & 99 \\
\hline Baise & Guangxi & NEC & 46 & 4 & 17 & 85 & 51 & 5 & 12 & 83 & 53 & 3 & 29 & 90 \\
\hline Beihai & Guangxi & NEC & 34 & 3 & 16 & 63 & 41 & 8 & 12 & 87 & 36 & 3 & 18 & 79 \\
\hline Chongzuo & Guangxi & NEC & 42 & 4 & 11 & 73 & 53 & 7 & 13 & 96 & 47 & 4 & 23 & 88 \\
\hline Fangchenggang & Guangxi & NEC & 34 & 3 & 15 & 63 & 44 & 8 & 12 & 100 & 40 & 3 & 22 & 81 \\
\hline Guigang & Guangxi & NEC & 61 & 4 & 32 & 105 & 60 & 10 & 19 & 122 & 48 & 3 & 17 & 85 \\
\hline Guilin & Guangxi & NEC & 43 & 3 & 26 & 63 & 58 & 6 & 17 & 86 & 68 & 4 & 33 & 112 \\
\hline Hechi & Guangxi & NEC & 47 & 4 & 30 & 79 & 61 & 6 & 16 & 98 & 55 & 5 & 17 & 139 \\
\hline Hezhou & Guangxi & NEC & 50 & 3 & 30 & 73 & 51 & 7 & 17 & 85 & 46 & 4 & 7 & 79 \\
\hline Laibin & Guangxi & NEC & 59 & 4 & 29 & 99 & 67 & 9 & 23 & 116 & 58 & 4 & 16 & 102 \\
\hline Liuzhou & Guangxi & NEC & 56 & 4 & 31 & 110 & 70 & 9 & 24 & 112 & 66 & 5 & 28 & 116 \\
\hline Nanning & Guangxi & NEC & 58 & 8 & 19 & 135 & 69 & 12 & 13 & 151 & 53 & 5 & 18 & 125 \\
\hline Qinzhou & Guangxi & NEC & 44 & 4 & 20 & 78 & 49 & 9 & 13 & 111 & 44 & 4 & 24 & 91 \\
\hline Wuzhou & Guangxi & NEC & 55 & 4 & 31 & 90 & 49 & 7 & 19 & 83 & 51 & 3 & 20 & 78 \\
\hline Yulin & Guangxi & NEC & 45 & 4 & 23 & 78 & 45 & 7 & 17 & 91 & 41 & 3 & 12 & 78 \\
\hline
\end{tabular}




\begin{tabular}{|c|c|c|c|c|c|c|c|c|c|c|c|c|c|c|}
\hline Golog & Qinghai & NEC & 77 & 7 & 34 & 133 & 86 & 8 & 53 & 122 & 53 & 5 & 16 & 120 \\
\hline Haidong & Qinghai & NEC & 37 & 4 & 0 & 75 & 49 & 7 & 20 & 126 & 33 & 2 & 8 & 50 \\
\hline Xining & Qinghai & NEC & 84 & 14 & 36 & 326 & 72 & 4 & 40 & 97 & 67 & 5 & 22 & 114 \\
\hline Ali & Tibet & NEC & 25 & 4 & 4 & 60 & 18 & 3 & 8 & 37 & 16 & 1 & 1 & 32 \\
\hline Qamdo & Tibet & NEC & 37 & 4 & 12 & 98 & 38 & 4 & 18 & 81 & 37 & 3 & 0 & 67 \\
\hline Lhasa & Tibet & NEC & 44 & 4 & 24 & 99 & 44 & 3 & 25 & 72 & 46 & 3 & 27 & 77 \\
\hline Nyingchi & Tibet & NEC & 15 & 1 & 6 & 30 & 12 & 1 & 7 & 22 & 14 & 1 & 8 & 25 \\
\hline Naqu & Tibet & NEC & 84 & 11 & 19 & 166 & 110 & 16 & 48 & 241 & 105 & 5 & 68 & 159 \\
\hline Shigatse & Tibet & NEC & 24 & 2 & 11 & 53 & 23 & 2 & 13 & 41 & 21 & 1 & 8 & 46 \\
\hline Lhoka & Tibet & NEC & 29 & 3 & 7 & 53 & 22 & 2 & 9 & 43 & 26 & 2 & 12 & 52 \\
\hline
\end{tabular}

132

9

1

${ }^{a}$ EC and NEC denote emission control and non-emission control regions, respectively, of which the latter means regions without implementation of emission control measures.

$\mathrm{b}$ The pre-Parade Blue, Parade Blue, and post-Parade Blue periods indicate the periods of 1-19 August, 20 August-3 September, and 4-30 September 2015, respectively. 
144 Table S4. Summary of daily average $\mathrm{NO}_{2}$ concentrations $\left(\mu \mathrm{g} \mathrm{m}^{-3}\right)$ during the pre-Parade Blue, Parade Blue and post-Parade Blue periods in the 145291 cities across China

\begin{tabular}{|c|c|c|c|c|c|c|c|c|c|c|c|c|c|c|}
\hline \multirow{2}{*}{ City } & \multirow{2}{*}{ Province } & \multirow{2}{*}{ Region $^{a}$} & \multicolumn{4}{|c|}{ Pre-Parade Blue period $^{\mathrm{b}}$} & \multicolumn{4}{|c|}{ Parade Blue period $^{\mathrm{b}}$} & \multicolumn{4}{|c|}{ Post-Parade Blue period ${ }^{\mathrm{b}}$} \\
\hline & & & Mean & $\mathrm{SE}$ & Min & Max & Mean & SE & Min & Max & Mean & SE & Min & Max \\
\hline Beijing & Municipality & EC & 34 & 1 & 26 & 44 & 21 & 1 & 17 & 24 & 42 & 2 & 20 & 63 \\
\hline Tianjing & Municipality & $\mathrm{EC}$ & 26 & 2 & 14 & 45 & 21 & 2 & 12 & 33 & 25 & 2 & 11 & 41 \\
\hline Baoding & Hebei & $\mathrm{EC}$ & 34 & 2 & 23 & 51 & 25 & 2 & 15 & 40 & 44 & 3 & 16 & 73 \\
\hline Cangzhou & Hebei & EC & 34 & 1 & 26 & 44 & 21 & 1 & 17 & 24 & 41 & 3 & 21 & 87 \\
\hline Chengde & Hebei & EC & 27 & 1 & 21 & 35 & 21 & 1 & 14 & 26 & 28 & 1 & 16 & 41 \\
\hline Handan & Hebei & EC & 38 & 3 & 22 & 70 & 36 & 3 & 20 & 54 & 45 & 3 & 15 & 76 \\
\hline Hengshui & Hebei & $\mathrm{EC}$ & 29 & 2 & 18 & 59 & 27 & 1 & 18 & 33 & 44 & 3 & 20 & 77 \\
\hline Langfang & Hebei & $\mathrm{EC}$ & 42 & 1 & 30 & 55 & 36 & 2 & 21 & 55 & 54 & 3 & 26 & 89 \\
\hline Qinhuangdao & Hebei & EC & 41 & 2 & 17 & 53 & 35 & 3 & 18 & 52 & 35 & 2 & 19 & 58 \\
\hline Shijiazhuang & Hebei & EC & 29 & 2 & 15 & 56 & 22 & 3 & 7 & 37 & 48 & 4 & 9 & 79 \\
\hline Tangshan & Hebei & EC & 44 & 2 & 32 & 76 & 45 & 4 & 10 & 74 & 58 & 3 & 23 & 96 \\
\hline Xingtai & Hebei & EC & 50 & 2 & 34 & 63 & 38 & 4 & 14 & 74 & 54 & 3 & 22 & 81 \\
\hline Zhangjiakou & Hebei & $\mathrm{EC}$ & 17 & 1 & 11 & 24 & 24 & 2 & 14 & 32 & 25 & 1 & 16 & 34 \\
\hline Changzhi & Shanxi & $\mathrm{EC}$ & 25 & 2 & 10 & 41 & 31 & 1 & 22 & 41 & 27 & 2 & 12 & 55 \\
\hline Datong & Shanxi & EC & 19 & 1 & 9 & 30 & 16 & 1 & 11 & 23 & 23 & 2 & 8 & 41 \\
\hline Jincheng & Shanxi & EC & 29 & 2 & 10 & 46 & 29 & 2 & 15 & 38 & 30 & 2 & 11 & 43 \\
\hline Jinzhong & Shanxi & EC & 22 & 1 & 15 & 31 & 20 & 1 & 12 & 29 & 24 & 1 & 11 & 36 \\
\hline Linfen & Shanxi & EC & 20 & 1 & 14 & 25 & 19 & 1 & 12 & 32 & 23 & 1 & 12 & 35 \\
\hline Lvliang & Shanxi & $\mathrm{EC}$ & 22 & 1 & 14 & 34 & 18 & 1 & 13 & 23 & 30 & 2 & 16 & 56 \\
\hline Shuozhou & Shanxi & EC & 31 & 2 & 13 & 45 & 31 & 1 & 20 & 39 & 31 & 2 & 15 & 65 \\
\hline Taiyuan & Shanxi & EC & 30 & 1 & 22 & 41 & 28 & 2 & 20 & 41 & 34 & 2 & 17 & 51 \\
\hline
\end{tabular}




\begin{tabular}{|c|c|c|c|c|c|c|c|c|c|c|c|c|c|}
\hline Xinzhou & Shanxi & EC & 33 & 2 & 16 & 48 & 29 & 2 & 16 & 37 & 34 & 2 & 14 \\
\hline Yangquan & Shanxi & $\mathrm{EC}$ & 24 & 1 & 12 & 32 & 23 & 2 & 11 & 32 & 31 & 1 & 17 \\
\hline Yuncheng & Shanxi & $\mathrm{EC}$ & 13 & 2 & 4 & 30 & 20 & 2 & 10 & 33 & 21 & 2 & 8 \\
\hline Binzhou & Shandong & EC & 27 & 2 & 16 & 49 & 28 & 3 & 15 & 55 & 35 & 2 & 14 \\
\hline Dezhou & Shandong & EC & 29 & 2 & 19 & 46 & 30 & 2 & 16 & 42 & 40 & 3 & 17 \\
\hline Dongying & Shandong & EC & 27 & 2 & 9 & 43 & 22 & 3 & 8 & 40 & 34 & 3 & 7 \\
\hline Jinan & Shandong & EC & 35 & 2 & 20 & 49 & 41 & 2 & 26 & 54 & 54 & 3 & 34 \\
\hline Jining & Shandong & $\mathrm{EC}$ & 31 & 2 & 13 & 50 & 37 & 3 & 24 & 67 & 43 & 2 & 25 \\
\hline Laiwu & Shandong & $\mathrm{EC}$ & 29 & 2 & 12 & 47 & 38 & 2 & 23 & 54 & 46 & 2 & 24 \\
\hline Liaocheng & Shandong & $\mathrm{EC}$ & 29 & 2 & 18 & 43 & 27 & 3 & 10 & 44 & 40 & 2 & 20 \\
\hline Linyi & Shandong & EC & 33 & 2 & 16 & 54 & 39 & 3 & 27 & 62 & 41 & 3 & 18 \\
\hline Qingdao & Shandong & EC & 19 & 1 & 11 & 35 & 28 & 1 & 16 & 36 & 29 & 2 & 12 \\
\hline Rizhao & Shandong & $\mathrm{EC}$ & 20 & 2 & 5 & 39 & 28 & 3 & 16 & 44 & 33 & 3 & 7 \\
\hline Tai'an & Shandong & $\mathrm{EC}$ & 30 & 2 & 17 & 47 & 38 & 2 & 22 & 50 & 38 & 2 & 22 \\
\hline Weihai & Shandong & EC & 15 & 1 & 6 & 28 & 14 & 1 & 7 & 22 & 18 & 1 & 5 \\
\hline Weifang & Shandong & EC & 25 & 2 & 14 & 41 & 33 & 2 & 22 & 48 & 33 & 2 & 14 \\
\hline Yantai & Shandong & EC & 23 & 1 & 12 & 33 & 27 & 2 & 17 & 42 & 31 & 2 & 12 \\
\hline Zaozhuang & Shandong & EC & 26 & 2 & 15 & 42 & 29 & 1 & 19 & 38 & 31 & 2 & 14 \\
\hline Zibo & Shandong & $\mathrm{EC}$ & 52 & 2 & 31 & 68 & 55 & 3 & 38 & 70 & 60 & 3 & 38 \\
\hline Alxa League & Inner Mongolia & EC & 7 & 0 & 5 & 10 & 8 & 1 & 4 & 11 & 17 & 1 & 9 \\
\hline Bayannur & Inner Mongolia & $\mathrm{EC}$ & 19 & 1 & 14 & 24 & 22 & 2 & 13 & 39 & 21 & 1 & 13 \\
\hline Baotou & Inner Mongolia & EC & 34 & 2 & 20 & 49 & 33 & 2 & 18 & 43 & 37 & 2 & 14 \\
\hline Chifeng & Inner Mongolia & $\mathrm{EC}$ & 16 & 1 & 10 & 27 & 17 & 0 & 14 & 20 & 17 & 1 & 10 \\
\hline Ordos & Inner Mongolia & $\mathrm{EC}$ & 19 & 1 & 9 & 30 & 18 & 1 & 12 & 26 & 18 & 1 & 9 \\
\hline Hohhot & Inner Mongolia & $\mathrm{EC}$ & 32 & 1 & 21 & 43 & 32 & 2 & 18 & 42 & 34 & 3 & 13 \\
\hline Hulun Buir & Inner Mongolia & EC & 20 & 2 & 7 & 37 & 19 & 1 & 11 & 27 & 17 & 1 & 7 \\
\hline
\end{tabular}




\begin{tabular}{|c|c|c|c|c|c|c|c|c|c|c|c|c|c|}
\hline Tongliao & Inner Mongolia & EC & 16 & 1 & 10 & 25 & 13 & 1 & 8 & 20 & 20 & 1 & 7 \\
\hline Wuhai & Inner Mongolia & EC & 17 & 1 & 11 & 24 & 17 & 1 & 11 & 24 & 25 & 2 & 9 \\
\hline Hinggan League & Inner Mongolia & $\mathrm{EC}$ & 14 & 1 & 6 & 22 & 10 & 1 & 7 & 16 & 14 & 1 & 9 \\
\hline Anyang & Henan & EC & 30 & 2 & 18 & 47 & 32 & 2 & 20 & 42 & 46 & 2 & 19 \\
\hline Hebi & Henan & EC & 27 & 3 & 9 & 48 & 24 & 2 & 16 & 39 & 48 & 3 & 16 \\
\hline Jiaozuo & Henan & EC & 29 & 2 & 21 & 47 & 30 & 2 & 17 & 40 & 36 & 2 & 16 \\
\hline Kaifeng & Henan & EC & 25 & 2 & 15 & 49 & 24 & 2 & 14 & 36 & 36 & 2 & 19 \\
\hline Luoyang & Henan & EC & 23 & 1 & 15 & 41 & 25 & 2 & 13 & 33 & 35 & 2 & 16 \\
\hline Luohe & Henan & $\mathrm{EC}$ & 18 & 2 & 9 & 33 & 23 & 2 & 12 & 35 & 31 & 2 & 13 \\
\hline Nanyang & Henan & EC & 16 & 1 & 8 & 25 & 21 & 1 & 14 & 28 & 22 & 2 & 8 \\
\hline Pingdingshan & Henan & EC & 27 & 2 & 14 & 41 & 33 & 2 & 23 & 43 & 38 & 2 & 22 \\
\hline Sanmenxia & Henan & EC & 23 & 2 & 15 & 43 & 23 & 3 & 14 & 49 & 28 & 1 & 16 \\
\hline Shangqiu & Henan & EC & 16 & 1 & 8 & 31 & 21 & 2 & 11 & 39 & 28 & 2 & 14 \\
\hline Xinyang & Henan & EC & 19 & 1 & 12 & 30 & 28 & 1 & 22 & 32 & 29 & 1 & 15 \\
\hline Zhengzhou & Henan & EC & 39 & 3 & 23 & 73 & 45 & 3 & 24 & 69 & 56 & 3 & 26 \\
\hline Zhoukou & Henan & EC & 11 & 1 & 7 & 17 & 13 & 1 & 8 & 19 & 18 & 1 & 9 \\
\hline Zhumadian & Henan & EC & 22 & 2 & 8 & 34 & 24 & 1 & 16 & 32 & 25 & 1 & 13 \\
\hline Anshan & Liaoning & NEC & 18 & 2 & 11 & 33 & 27 & 1 & 16 & 31 & 31 & 2 & 11 \\
\hline Benxi & Liaoning & NEC & 19 & 1 & 11 & 30 & 25 & 1 & 14 & 34 & 36 & 2 & 17 \\
\hline Chaoyang & Liaoning & NEC & 12 & 1 & 7 & 16 & 13 & 1 & 8 & 17 & 14 & 1 & 8 \\
\hline Dalian & Liaoning & NEC & 20 & 1 & 14 & 25 & 23 & 1 & 17 & 31 & 30 & 1 & 15 \\
\hline Dandong & Liaoning & NEC & 29 & 8 & 1 & 108 & 15 & 1 & 7 & 20 & 20 & 1 & 12 \\
\hline Fushun & Liaoning & NEC & 15 & 1 & 10 & 22 & 18 & 1 & 8 & 24 & 30 & 2 & 12 \\
\hline Fuxin & Liaoning & NEC & 28 & 1 & 18 & 38 & 25 & 1 & 15 & 35 & 31 & 1 & 15 \\
\hline Huludao & Liaoning & NEC & 23 & 2 & 11 & 44 & 28 & 2 & 15 & 41 & 33 & 2 & 14 \\
\hline Jinzhou & Liaoning & NEC & 26 & 1 & 18 & 36 & 28 & 1 & 21 & 36 & 34 & 1 & 20 \\
\hline
\end{tabular}




\begin{tabular}{|c|c|c|c|c|c|c|c|c|c|c|c|c|c|}
\hline Liaoyang & Liaoning & NEC & 15 & 1 & 6 & 31 & 26 & 1 & 15 & 32 & 30 & 2 & 6 \\
\hline Panjin & Liaoning & NEC & 13 & 2 & 4 & 32 & 24 & 1 & 17 & 32 & 27 & 2 & 13 \\
\hline Shenyang & Liaoning & NEC & 31 & 2 & 20 & 49 & 35 & 2 & 27 & 48 & 44 & 2 & 25 \\
\hline Tieling & Liaoning & NEC & 20 & 1 & 13 & 26 & 23 & 1 & 14 & 29 & 29 & 1 & 16 \\
\hline Wafangdian & Liaoning & NEC & 17 & 1 & 11 & 24 & 21 & 1 & 15 & 33 & 31 & 1 & 18 \\
\hline Yingkou & Liaoning & NEC & 16 & 1 & 8 & 27 & 22 & 1 & 15 & 33 & 30 & 2 & 16 \\
\hline Baicheng & Jilin & NEC & 12 & 1 & 8 & 21 & 9 & 1 & 4 & 13 & 11 & 1 & 7 \\
\hline Baishan & Jilin & NEC & 14 & 0 & 9 & 18 & 16 & 1 & 12 & 19 & 21 & 1 & 13 \\
\hline Changchun & Jilin & NEC & 28 & 2 & 17 & 44 & 33 & 2 & 22 & 45 & 42 & 2 & 22 \\
\hline Jilin & Jilin & NEC & 20 & 1 & 13 & 28 & 24 & 1 & 16 & 31 & 31 & 1 & 25 \\
\hline Liaoyuan & Jilin & NEC & 15 & 2 & 3 & 30 & 15 & 1 & 8 & 24 & 26 & 1 & 11 \\
\hline Siping & Jilin & NEC & 17 & 2 & 4 & 33 & 23 & 3 & 13 & 62 & 32 & 2 & 12 \\
\hline Songyuan & Jilin & NEC & 16 & 1 & 9 & 24 & 14 & 1 & 6 & 22 & 26 & 2 & 10 \\
\hline Daqing & Heilongjiang & NEC & 16 & 1 & 12 & 23 & 16 & 1 & 11 & 24 & 21 & 1 & 10 \\
\hline Daxinganling & Heilongjiang & NEC & 11 & 1 & 8 & 15 & 15 & 1 & 10 & 20 & 11 & 1 & 6 \\
\hline Harbin & Heilongjiang & NEC & 31 & 2 & 21 & 51 & 31 & 2 & 20 & 42 & 37 & 2 & 18 \\
\hline Hegang & Heilongjiang & NEC & 6 & 1 & 3 & 10 & 4 & 0 & 2 & 6 & 9 & 1 & 4 \\
\hline Heihe & Heilongjiang & NEC & 11 & 1 & 5 & 15 & 9 & 1 & 7 & 14 & 12 & 1 & 6 \\
\hline Jixi & Heilongjiang & NEC & 16 & 1 & 11 & 21 & 13 & 1 & 8 & 19 & 18 & 1 & 9 \\
\hline Jiamusi & Heilongjiang & NEC & 18 & 1 & 8 & 27 & 15 & 2 & 8 & 31 & 22 & 2 & 5 \\
\hline Mudanjiang & Heilongjiang & NEC & 24 & 1 & 16 & 33 & 19 & 1 & 12 & 25 & 20 & 1 & 13 \\
\hline Qitaihe & Heilongjiang & NEC & 12 & 0 & 10 & 16 & 11 & 1 & 7 & 15 & 13 & 1 & 5 \\
\hline Qiqihar & Heilongjiang & NEC & 15 & 1 & 8 & 19 & 11 & 1 & 7 & 16 & 18 & 1 & 10 \\
\hline Shuangyashan & Heilongjiang & NEC & 15 & 0 & 12 & 19 & 15 & 1 & 9 & 18 & 18 & 1 & 13 \\
\hline Suihua & Heilongjiang & NEC & 20 & 2 & 10 & 41 & 15 & 1 & 10 & 24 & 15 & 1 & 7 \\
\hline Ankang & Shaanxi & NEC & 6 & 1 & 3 & 11 & 7 & 1 & 3 & 11 & 10 & 1 & 5 \\
\hline
\end{tabular}




\begin{tabular}{|c|c|c|c|c|c|c|c|c|c|c|c|c|c|}
\hline Baoji & Shaanxi & NEC & 23 & 1 & 16 & 34 & 28 & 1 & 16 & 33 & 28 & 1 & 17 \\
\hline Hanzhong & Shaanxi & NEC & 15 & 1 & 9 & 22 & 22 & 1 & 13 & 27 & 23 & 1 & 10 \\
\hline Shangluo & Shaanxi & NEC & 15 & 1 & 10 & 22 & 19 & 1 & 13 & 24 & 21 & 1 & 15 \\
\hline Tongchuan & Shaanxi & NEC & 24 & 2 & 15 & 47 & 26 & 1 & 17 & 32 & 27 & 1 & 15 \\
\hline Weinan & Shaanxi & NEC & 29 & 1 & 22 & 43 & 39 & 2 & 29 & 54 & 37 & 2 & 22 \\
\hline Xi'an & Shaanxi & NEC & 36 & 2 & 24 & 55 & 44 & 2 & 29 & 57 & 38 & 2 & 21 \\
\hline Xianyang & Shaanxi & NEC & 23 & 1 & 13 & 36 & 27 & 1 & 19 & 34 & 30 & 2 & 16 \\
\hline Yan'an & Shaanxi & NEC & 35 & 1 & 23 & 40 & 40 & 1 & 33 & 53 & 35 & 1 & 27 \\
\hline Yulin & Shaanxi & NEC & 27 & 2 & 12 & 43 & 33 & 3 & 14 & 56 & 26 & 2 & 15 \\
\hline Baiying & Gansu & NEC & 25 & 2 & 12 & 40 & 28 & 3 & 4 & 42 & 22 & 2 & 6 \\
\hline Dingxi & Gansu & NEC & 20 & 2 & 9 & 40 & 20 & 1 & 12 & 30 & 18 & 1 & 10 \\
\hline Gannan & Gansu & NEC & 17 & 2 & 8 & 40 & 14 & 1 & 10 & 19 & 15 & 1 & 10 \\
\hline Jiayuguan & Gansu & NEC & 25 & 1 & 13 & 37 & 19 & 1 & 13 & 29 & 28 & 1 & 15 \\
\hline Jiuquan & Gansu & NEC & 23 & 2 & 8 & 37 & 25 & 2 & 12 & 38 & 26 & 1 & 14 \\
\hline Lanzhou & Gansu & NEC & 38 & 2 & 20 & 53 & 42 & 3 & 25 & 59 & 42 & 2 & 23 \\
\hline Linxia & Gansu & NEC & 26 & 2 & 12 & 49 & 31 & 2 & 20 & 49 & 25 & 2 & 7 \\
\hline Pingliang & Gansu & NEC & 41 & 1 & 28 & 50 & 50 & 2 & 38 & 62 & 43 & 1 & 29 \\
\hline Qingyang & Gansu & NEC & 10 & 1 & 5 & 16 & 14 & 1 & 9 & 20 & 14 & 1 & 7 \\
\hline Tianshui & Gansu & NEC & 16 & 1 & 10 & 22 & 14 & 1 & 6 & 19 & 20 & 1 & 14 \\
\hline Wuwei & Gansu & NEC & 22 & 1 & 13 & 36 & 23 & 2 & 14 & 35 & 21 & 2 & 11 \\
\hline Zhangye & Gansu & NEC & 18 & 1 & 10 & 26 & 15 & 1 & 8 & 21 & 20 & 1 & 11 \\
\hline Guyuan & Ningxia & NEC & 18 & 2 & 9 & 30 & 22 & 2 & 11 & 31 & 17 & 1 & 9 \\
\hline Shizuishan & Ningxia & NEC & 17 & 1 & 12 & 33 & 20 & 1 & 13 & 30 & 23 & 1 & 11 \\
\hline Wuzhong & Ningxia & NEC & 17 & 1 & 9 & 29 & 18 & 2 & 5 & 28 & 16 & 2 & 6 \\
\hline Yinchuan & Ningxia & NEC & 27 & 2 & 13 & 48 & 29 & 3 & 16 & 45 & 25 & 2 & 10 \\
\hline Zhongwei & Ningxia & NEC & 13 & 1 & 7 & 18 & 17 & 1 & 9 & 22 & 15 & 1 & 6 \\
\hline
\end{tabular}




\begin{tabular}{|c|c|c|c|c|c|c|c|c|c|c|c|c|c|}
\hline Aksu & Sinkiang & NEC & 23 & 2 & 12 & 42 & 21 & 2 & 11 & 38 & 22 & 2 & 8 \\
\hline Hami & Sinkiang & NEC & 11 & 1 & 4 & 21 & 9 & 1 & 4 & 19 & 12 & 1 & 5 \\
\hline Hotan & Sinkiang & NEC & 21 & 2 & 11 & 35 & 18 & 2 & 10 & 29 & 23 & 2 & 11 \\
\hline Kashgar & Sinkiang & NEC & 22 & 2 & 10 & 36 & 19 & 2 & 9 & 36 & 23 & 2 & 11 \\
\hline Karamay & Sinkiang & NEC & 15 & 1 & 5 & 28 & 14 & 1 & 6 & 28 & 14 & 1 & 6 \\
\hline Kizilsu & Sinkiang & NEC & 9 & 1 & 1 & 19 & 9 & 1 & 6 & 15 & 9 & 1 & 1 \\
\hline Shihezi & Sinkiang & NEC & 28 & 3 & 7 & 64 & 22 & 3 & 6 & 38 & 26 & 2 & 10 \\
\hline Urumchi & Sinkiang & NEC & 41 & 2 & 20 & 58 & 38 & 2 & 24 & 50 & 35 & 3 & 15 \\
\hline Wujiaqu & Sinkiang & NEC & 30 & 3 & 9 & 53 & 24 & 3 & 10 & 49 & 21 & 2 & 4 \\
\hline Yili & Sinkiang & NEC & 25 & 2 & 11 & 34 & 24 & 2 & 12 & 34 & 26 & 2 & 10 \\
\hline Ezhou & Hubei & NEC & 13 & 1 & 8 & 24 & 19 & 1 & 11 & 30 & 21 & 1 & 10 \\
\hline Huanggang & Hubei & NEC & 14 & 2 & 4 & 23 & 14 & 1 & 6 & 26 & 20 & 2 & 8 \\
\hline Jingmen & Hubei & NEC & 18 & 1 & 12 & 38 & 27 & 2 & 17 & 38 & 25 & 2 & 13 \\
\hline Shiyan & Hubei & NEC & 12 & 1 & 8 & 19 & 13 & 0 & 10 & 16 & 15 & 1 & 8 \\
\hline Wuhan & Hubei & NEC & 27 & 2 & 11 & 52 & 41 & 4 & 18 & 64 & 40 & 3 & 19 \\
\hline Xianning & Hubei & NEC & 12 & 0 & 9 & 16 & 15 & 1 & 11 & 19 & 16 & 1 & 9 \\
\hline Xiangyang & Hubei & NEC & 17 & 1 & 10 & 34 & 23 & 1 & 16 & 31 & 30 & 2 & 16 \\
\hline Xiaogan & Hubei & NEC & 5 & 1 & 2 & 12 & 9 & 1 & 4 & 20 & 17 & 2 & 6 \\
\hline Yichang & Hubei & NEC & 24 & 1 & 19 & 32 & 26 & 1 & 23 & 32 & 33 & 1 & 23 \\
\hline Anqing & Anhui & NEC & 21 & 2 & 12 & 37 & 21 & 2 & 14 & 46 & 21 & 1 & 14 \\
\hline Bengbu & Anhui & NEC & 24 & 2 & 11 & 40 & 32 & 2 & 20 & 42 & 33 & 2 & 21 \\
\hline Bozhou & Anhui & NEC & 33 & 1 & 24 & 45 & 46 & 2 & 35 & 72 & 41 & 2 & 27 \\
\hline Chuzhou & Anhui & NEC & 29 & 2 & 18 & 41 & 22 & 1 & 11 & 30 & 22 & 2 & 12 \\
\hline Fuyang & Anhui & NEC & 28 & 1 & 21 & 46 & 43 & 3 & 30 & 64 & 41 & 2 & 25 \\
\hline Hefei & Anhui & NEC & 24 & 2 & 14 & 38 & 31 & 3 & 19 & 58 & 29 & 2 & 18 \\
\hline Huaibei & Anhui & NEC & 19 & 1 & 14 & 25 & 24 & 1 & 18 & 32 & 28 & 2 & 18 \\
\hline
\end{tabular}




\begin{tabular}{|c|c|c|c|c|c|c|c|c|c|c|c|c|c|}
\hline Huainan & Anhui & NEC & 18 & 1 & 11 & 29 & 20 & 1 & 13 & 26 & 23 & 1 & 14 \\
\hline Huangshan & Anhui & NEC & 9 & 0 & 8 & 10 & 9 & 0 & 8 & 10 & 10 & 0 & 8 \\
\hline Lu'an & Anhui & NEC & 15 & 1 & 8 & 27 & 15 & 1 & 9 & 21 & 15 & 1 & 7 \\
\hline Ma'anshan & Anhui & NEC & 20 & 1 & 10 & 30 & 21 & 1 & 15 & 28 & 19 & 1 & 6 \\
\hline Suzhou & Anhui & NEC & 18 & 0 & 15 & 21 & 19 & 1 & 16 & 26 & 21 & 1 & 16 \\
\hline Tongling & Anhui & NEC & 25 & 2 & 15 & 35 & 31 & 2 & 18 & 47 & 30 & 1 & 18 \\
\hline Wuhu & Anhui & NEC & 38 & 1 & 30 & 51 & 38 & 2 & 29 & 49 & 40 & 2 & 24 \\
\hline Changzhou & Jiangsu & NEC & 33 & 3 & 16 & 70 & 34 & 3 & 15 & 51 & 33 & 2 & 20 \\
\hline Huaian & Jiangsu & NEC & 12 & 1 & 3 & 22 & 15 & 2 & 5 & 30 & 16 & 1 & 6 \\
\hline Lianyungang & Jiangsu & NEC & 17 & 1 & 9 & 27 & 24 & 1 & 16 & 32 & 25 & 2 & 10 \\
\hline Nanjing & Jiangsu & NEC & 36 & 2 & 19 & 54 & 39 & 3 & 25 & 61 & 44 & 2 & 20 \\
\hline Nantong & Jiangsu & NEC & 24 & 3 & 7 & 49 & 25 & 3 & 8 & 50 & 24 & 1 & 12 \\
\hline Suzhou & Jiangsu & NEC & 31 & 2 & 18 & 46 & 42 & 2 & 32 & 57 & 42 & 2 & 27 \\
\hline Suqian & Jiangsu & NEC & 26 & 2 & 8 & 38 & 27 & 1 & 20 & 39 & 30 & 2 & 10 \\
\hline Taizhou & Jiangsu & NEC & 19 & 2 & 8 & 33 & 20 & 1 & 14 & 34 & 23 & 1 & 15 \\
\hline Wuxi & Jiangsu & NEC & 27 & 2 & 15 & 51 & 34 & 1 & 27 & 48 & 31 & 1 & 18 \\
\hline Xuzhou & Jiangsu & NEC & 26 & 2 & 12 & 43 & 33 & 2 & 21 & 48 & 35 & 2 & 18 \\
\hline Yanchen & Jiangsu & NEC & 17 & 1 & 8 & 26 & 22 & 1 & 14 & 32 & 21 & 1 & 11 \\
\hline Yangzhou & Jiangsu & NEC & 23 & 2 & 7 & 48 & 18 & 1 & 12 & 28 & 21 & 1 & 9 \\
\hline Zhenjiang & Jiangsu & NEC & 27 & 2 & 16 & 44 & 35 & 2 & 18 & 49 & 29 & 1 & 18 \\
\hline Shanghai & Municipality & NEC & 21 & 2 & 9 & 33 & 36 & 3 & 19 & 54 & 29 & 1 & 17 \\
\hline Hangzhou & Zhejiang & NEC & 29 & 2 & 11 & 48 & 33 & 2 & 20 & 45 & 40 & 2 & 20 \\
\hline Huzhou & Zhejiang & NEC & 25 & 2 & 9 & 40 & 32 & 2 & 22 & 49 & 30 & 3 & 12 \\
\hline Jiaxing & Zhejiang & NEC & 19 & 2 & 6 & 30 & 29 & 2 & 19 & 51 & 33 & 2 & 15 \\
\hline Jinhua & Zhejiang & NEC & 20 & 1 & 9 & 31 & 25 & 2 & 8 & 38 & 32 & 2 & 14 \\
\hline Lishui & Zhejiang & NEC & 14 & 1 & 5 & 20 & 14 & 1 & 10 & 19 & 20 & 1 & 10 \\
\hline
\end{tabular}




\begin{tabular}{|c|c|c|c|c|c|c|c|c|c|c|c|c|c|c|}
\hline Ningbo & Zhejiang & NEC & 21 & 2 & 8 & 38 & 29 & 2 & 14 & 42 & 36 & 3 & 13 & 66 \\
\hline Quzhou & Zhejiang & NEC & 20 & 1 & 9 & 31 & 26 & 2 & 8 & 41 & 28 & 1 & 16 & 45 \\
\hline Shaoxing & Zhejiang & NEC & 26 & 2 & 11 & 39 & 38 & 1 & 28 & 49 & 40 & 2 & 20 & 64 \\
\hline Taizhou & Zhejiang & NEC & 11 & 1 & 1 & 20 & 15 & 2 & 7 & 25 & 18 & 1 & 4 & 28 \\
\hline Wenjiang & Zhejiang & NEC & 25 & 3 & 2 & 44 & 29 & 3 & 10 & 45 & 34 & 2 & 12 & 55 \\
\hline Zhoushan & Zhejiang & NEC & 11 & 1 & 3 & 24 & 14 & 1 & 5 & 20 & 13 & 1 & 5 & 23 \\
\hline Fuzhou & Jiangxi & NEC & 10 & 1 & 5 & 14 & 12 & 1 & 6 & 19 & 13 & 1 & 7 & 22 \\
\hline Ganzhou & Jiangxi & NEC & 13 & 1 & 7 & 18 & 15 & 1 & 10 & 20 & 16 & 1 & 11 & 22 \\
\hline Ji'an & Jiangxi & NEC & 9 & 1 & 5 & 18 & 14 & 1 & 8 & 22 & 15 & 1 & 7 & 27 \\
\hline Jingdezhen & Jiangxi & NEC & 12 & 1 & 5 & 32 & 12 & 1 & 6 & 18 & 13 & 1 & 8 & 23 \\
\hline Jiujiang & Jiangxi & NEC & 15 & 1 & 8 & 25 & 19 & 1 & 12 & 29 & 20 & 1 & 11 & 31 \\
\hline Nanchang & Jiangxi & NEC & 20 & 2 & 8 & 35 & 21 & 2 & 14 & 40 & 19 & 1 & 12 & 27 \\
\hline Pingxiang & Jiangxi & NEC & 10 & 1 & 5 & 15 & 15 & 1 & 10 & 18 & 14 & 1 & 7 & 21 \\
\hline Shangrao & Jiangxi & NEC & 14 & 1 & 8 & 21 & 18 & 1 & 12 & 28 & 21 & 1 & 13 & 28 \\
\hline Xinyu & Jiangxi & NEC & 11 & 1 & 5 & 16 & 12 & 1 & 7 & 18 & 14 & 1 & 9 & 22 \\
\hline Yichun & Jiangxi & NEC & 13 & 0 & 9 & 17 & 14 & 1 & 10 & 19 & 16 & 1 & 10 & 23 \\
\hline Yingtan & Jiangxi & NEC & 10 & 0 & 6 & 14 & 13 & 1 & 9 & 18 & 18 & 1 & 9 & 22 \\
\hline Changsha & Hunan & NEC & 19 & 1 & 9 & 31 & 27 & 1 & 16 & 33 & 26 & 2 & 15 & 48 \\
\hline Changde & Hunan & NEC & 13 & 1 & 6 & 24 & 11 & 1 & 8 & 16 & 13 & 1 & 7 & 22 \\
\hline Chenzhou & Hunan & NEC & 18 & 1 & 9 & 26 & 20 & 1 & 12 & 25 & 23 & 1 & 13 & 36 \\
\hline Huaihua & Hunan & NEC & 8 & 1 & 4 & 14 & 14 & 1 & 5 & 22 & 12 & 1 & 4 & 21 \\
\hline Loudi & Hunan & NEC & 11 & 1 & 6 & 16 & 12 & 1 & 8 & 18 & 10 & 0 & 4 & 15 \\
\hline Xiangtan & Hunan & NEC & 24 & 1 & 13 & 34 & 30 & 2 & 17 & 42 & 31 & 2 & 16 & 53 \\
\hline Yiyang & Hunan & NEC & 15 & 1 & 9 & 22 & 18 & 1 & 12 & 27 & 19 & 1 & 10 & 34 \\
\hline Yongzhou & Hunan & NEC & 19 & 1 & 14 & 25 & 21 & 1 & 14 & 28 & 24 & 1 & 13 & 32 \\
\hline Zhangjiajie & Hunan & NEC & 18 & 1 & 11 & 29 & 22 & 1 & 18 & 26 & 21 & 1 & 15 & 28 \\
\hline
\end{tabular}




\begin{tabular}{|c|c|c|c|c|c|c|c|c|c|c|c|c|c|c|}
\hline Zhuzhou & Hunan & NEC & 17 & 1 & 12 & 25 & 25 & 1 & 18 & 30 & 27 & 1 & 17 & 39 \\
\hline Dongguan & Guangdong & NEC & 31 & 2 & 20 & 45 & 31 & 2 & 24 & 49 & 28 & 2 & 4 & 46 \\
\hline Foshan & Guangdong & NEC & 32 & 2 & 20 & 44 & 41 & 1 & 33 & 49 & 29 & 1 & 18 & 44 \\
\hline Guangzhou & Guangdong & NEC & 35 & 1 & 23 & 47 & 38 & 2 & 28 & 48 & 41 & 2 & 21 & 60 \\
\hline Heyuan & Guangdong & NEC & 18 & 1 & 10 & 27 & 19 & 1 & 11 & 26 & 20 & 1 & 13 & 39 \\
\hline Huizhou & Guangdong & NEC & 19 & 1 & 10 & 27 & 17 & 1 & 13 & 25 & 20 & 2 & 8 & 39 \\
\hline Jiangmen & Guangdong & NEC & 14 & 1 & 9 & 20 & 25 & 1 & 19 & 34 & 20 & 2 & 8 & 37 \\
\hline Maoming & Guangdong & NEC & 10 & 1 & 6 & 14 & 12 & 1 & 9 & 15 & 10 & 0 & 6 & 15 \\
\hline Meizhou & Guangdong & NEC & 15 & 1 & 10 & 19 & 16 & 1 & 11 & 25 & 19 & 1 & 12 & 28 \\
\hline Qingyuan & Guangdong & NEC & 33 & 1 & 22 & 44 & 24 & 2 & 14 & 40 & 24 & 1 & 13 & 40 \\
\hline Shantou & Guangdong & NEC & 16 & 1 & 10 & 26 & 16 & 1 & 12 & 19 & 13 & 1 & 7 & 22 \\
\hline Shaoguan & Guangdong & NEC & 19 & 1 & 12 & 25 & 15 & 1 & 10 & 18 & 18 & 1 & 9 & 34 \\
\hline Shenzhen & Guangdong & NEC & 34 & 2 & 22 & 47 & 33 & 2 & 24 & 48 & 30 & 1 & 21 & 45 \\
\hline Zhujiang & Guangdong & NEC & 14 & 1 & 6 & 20 & 16 & 1 & 8 & 22 & 13 & 1 & 6 & 23 \\
\hline Zhaoqing & Guangdong & NEC & 20 & 1 & 13 & 30 & 26 & 2 & 12 & 41 & 23 & 1 & 15 & 35 \\
\hline Zhongshan & Guangdong & NEC & 15 & 1 & 8 & 31 & 24 & 2 & 14 & 33 & 19 & 1 & 9 & 31 \\
\hline Zhuhai & Guangdong & NEC & 17 & 1 & 10 & 28 & 25 & 1 & 19 & 31 & 19 & 1 & 10 & 28 \\
\hline Fuzhou & Fujian & NEC & 21 & 1 & 4 & 30 & 22 & 2 & 8 & 36 & 24 & 2 & 9 & 47 \\
\hline Longyan & Fujian & NEC & 17 & 1 & 9 & 28 & 17 & 1 & 13 & 20 & 20 & 1 & 10 & 31 \\
\hline Nanping & Fujian & NEC & 10 & 1 & 7 & 15 & 9 & 1 & 6 & 13 & 10 & 0 & 6 & 14 \\
\hline Ningde & Fujian & NEC & 13 & 1 & 3 & 22 & 14 & 1 & 8 & 19 & 17 & 1 & 7 & 31 \\
\hline Putian & Fujian & NEC & 15 & 1 & 3 & 23 & 13 & 1 & 5 & 19 & 13 & 1 & 4 & 20 \\
\hline Quanzhou & Fujian & NEC & 18 & 2 & 2 & 27 & 14 & 1 & 6 & 23 & 16 & 1 & 8 & 34 \\
\hline Sanming & Fujian & NEC & 22 & 1 & 12 & 29 & 20 & 1 & 11 & 31 & 23 & 1 & 15 & 33 \\
\hline Xiamen & Fujian & NEC & 26 & 1 & 15 & 41 & 22 & 1 & 17 & 28 & 19 & 1 & 11 & 34 \\
\hline Zhangzhou & Fujian & NEC & 18 & 1 & 10 & 22 & 19 & 1 & 16 & 24 & 22 & 1 & 13 & 38 \\
\hline
\end{tabular}




\begin{tabular}{|c|c|c|c|c|c|c|c|c|c|c|c|c|c|}
\hline Haikou & Hainan & NEC & 13 & 1 & 8 & 21 & 16 & 1 & 12 & 21 & 12 & 1 & 5 \\
\hline Sanya & Hainan & NEC & 11 & 1 & 7 & 19 & 14 & 1 & 9 & 20 & 13 & 1 & 4 \\
\hline Chongqing & Municipality & NEC & 29 & 2 & 20 & 48 & 46 & 2 & 35 & 57 & 37 & 1 & 22 \\
\hline Bazhong & Sichuan & NEC & 18 & 1 & 11 & 23 & 22 & 1 & 17 & 27 & 21 & 1 & 15 \\
\hline Chengdu & Sichuan & NEC & 39 & 3 & 15 & 63 & 54 & 4 & 25 & 81 & 43 & 2 & 26 \\
\hline Dazhou & Sichuan & NEC & 31 & 1 & 21 & 38 & 35 & 1 & 26 & 45 & 34 & 1 & 24 \\
\hline Deyang & Sichuan & NEC & 25 & 2 & 9 & 39 & 30 & 2 & 17 & 48 & 24 & 2 & 9 \\
\hline Guangyuan & Sichuan & NEC & 24 & 1 & 19 & 39 & 26 & 2 & 18 & 40 & 27 & 1 & 13 \\
\hline Leshan & Sichuan & NEC & 23 & 1 & 15 & 35 & 28 & 2 & 17 & 42 & 24 & 1 & 17 \\
\hline Luzhou & Sichuan & NEC & 20 & 1 & 11 & 30 & 28 & 2 & 12 & 41 & 23 & 1 & 10 \\
\hline Meishan & Sichuan & NEC & 19 & 1 & 10 & 34 & 25 & 2 & 15 & 39 & 22 & 1 & 14 \\
\hline Mianyang & Sichuan & NEC & 32 & 2 & 17 & 43 & 37 & 2 & 21 & 51 & 28 & 2 & 15 \\
\hline Nanchong & Sichuan & NEC & 20 & 2 & 11 & 31 & 29 & 1 & 18 & 36 & 33 & 2 & 21 \\
\hline Panzhihua & Sichuan & NEC & 25 & 1 & 21 & 35 & 27 & 1 & 23 & 35 & 24 & 1 & 18 \\
\hline Suining & Sichuan & NEC & 11 & 1 & 4 & 19 & 16 & 1 & 7 & 27 & 14 & 1 & 5 \\
\hline Ya'an & Sichuan & NEC & 22 & 1 & 16 & 35 & 21 & 1 & 16 & 23 & 24 & 1 & 16 \\
\hline Ziyang & Sichuan & NEC & 13 & 1 & 9 & 23 & 20 & 1 & 15 & 29 & 16 & 1 & 10 \\
\hline Zigong & Sichuan & NEC & 22 & 1 & 15 & 34 & 28 & 2 & 16 & 38 & 23 & 1 & 17 \\
\hline Baoshan & Yunnan & NEC & 14 & 0 & 10 & 18 & 15 & 1 & 10 & 19 & 16 & 1 & 11 \\
\hline Chuxiong & Yunnan & NEC & 16 & 1 & 8 & 22 & 23 & 2 & 7 & 34 & 14 & 1 & 7 \\
\hline Dali & Yunnan & NEC & 11 & 1 & 6 & 19 & 12 & 1 & 4 & 20 & 10 & 1 & 6 \\
\hline Dehong & Yunnan & NEC & 10 & 0 & 7 & 13 & 10 & 0 & 8 & 13 & 14 & 0 & 9 \\
\hline Honghe & Yunnan & NEC & 10 & 1 & 5 & 19 & 14 & 1 & 10 & 20 & 8 & 1 & 3 \\
\hline Kunming & Yunnan & NEC & 20 & 1 & 13 & 27 & 28 & 2 & 16 & 39 & 23 & 1 & 16 \\
\hline Lijiang & Yunnan & NEC & 11 & 0 & 8 & 15 & 13 & 0 & 11 & 17 & 11 & 1 & 5 \\
\hline Lincang & Yunnan & NEC & 10 & 0 & 7 & 15 & 11 & 1 & 9 & 17 & 10 & 1 & 4 \\
\hline
\end{tabular}




\begin{tabular}{|c|c|c|c|c|c|c|c|c|c|c|c|c|c|}
\hline Nujiang & Yunnan & NEC & 7 & 0 & 5 & 11 & 9 & 0 & 7 & 11 & 9 & 0 & 7 \\
\hline Qujing & Yunnan & NEC & 17 & 1 & 11 & 24 & 20 & 1 & 15 & 31 & 17 & 1 & 11 \\
\hline Wenshan & Yunnan & NEC & 12 & 1 & 7 & 19 & 17 & 1 & 11 & 25 & 11 & 0 & 7 \\
\hline Xishuangbanna & Yunnan & NEC & 9 & 0 & 7 & 14 & 11 & 1 & 7 & 14 & 12 & 1 & 6 \\
\hline Tuxi & Yunnan & NEC & 15 & 1 & 8 & 21 & 19 & 1 & 14 & 25 & 14 & 1 & 9 \\
\hline Zhaotong & Yunnan & NEC & 17 & 1 & 11 & 26 & 22 & 1 & 12 & 30 & 15 & 1 & 10 \\
\hline Anshun & Guizhou & NEC & 9 & 1 & 4 & 24 & 15 & 2 & 5 & 29 & 10 & 1 & 5 \\
\hline Bijie & Guizhou & NEC & 23 & 2 & 11 & 36 & 27 & 2 & 18 & 39 & 22 & 1 & 10 \\
\hline Guiyang & Guizhou & NEC & 20 & 1 & 13 & 32 & 29 & 2 & 16 & 44 & 22 & 1 & 15 \\
\hline Liupanshui & Guizhou & NEC & 23 & 1 & 16 & 40 & 31 & 2 & 19 & 49 & 23 & 1 & 18 \\
\hline Tongren & Guizhou & NEC & 14 & 1 & 9 & 18 & 18 & 1 & 13 & 21 & 18 & 1 & 10 \\
\hline Zunyi & Guizhou & NEC & 20 & 1 & 16 & 27 & 32 & 2 & 15 & 42 & 25 & 1 & 16 \\
\hline Baise & Guangxi & NEC & 12 & 1 & 8 & 19 & 9 & 1 & 3 & 18 & 10 & 1 & 5 \\
\hline Beihai & Guangxi & NEC & 10 & 1 & 6 & 16 & 9 & 1 & 6 & 14 & 10 & 1 & 6 \\
\hline Chongzuo & Guangxi & NEC & 13 & 1 & 6 & 24 & 16 & 1 & 8 & 25 & 12 & 1 & 5 \\
\hline Fangchenggang & Guangxi & NEC & 11 & 0 & 7 & 14 & 12 & 1 & 8 & 16 & 12 & 0 & 7 \\
\hline Guigang & Guangxi & NEC & 20 & 1 & 13 & 25 & 20 & 1 & 12 & 27 & 18 & 1 & 9 \\
\hline Guilin & Guangxi & NEC & 15 & 1 & 10 & 20 & 17 & 1 & 13 & 22 & 17 & 1 & 10 \\
\hline Hechi & Guangxi & NEC & 13 & 1 & 9 & 21 & 15 & 1 & 9 & 21 & 20 & 1 & 13 \\
\hline Hezhou & Guangxi & NEC & 9 & 0 & 5 & 13 & 8 & 1 & 5 & 14 & 8 & 1 & 4 \\
\hline Laibin & Guangxi & NEC & 14 & 1 & 8 & 20 & 16 & 1 & 8 & 26 & 15 & 1 & 10 \\
\hline Liuzhou & Guangxi & NEC & 19 & 1 & 13 & 30 & 18 & 1 & 9 & 27 & 19 & 1 & 11 \\
\hline Nanning & Guangxi & NEC & 25 & 2 & 15 & 45 & 28 & 2 & 17 & 45 & 25 & 1 & 17 \\
\hline Qinzhou & Guangxi & NEC & 15 & 1 & 8 & 22 & 16 & 1 & 11 & 24 & 16 & 1 & 12 \\
\hline Wuzhou & Guangxi & NEC & 19 & 1 & 14 & 24 & 17 & 1 & 11 & 28 & 18 & 1 & 11 \\
\hline Yulin & Guangxi & NEC & 15 & 1 & 9 & 25 & 17 & 1 & 10 & 27 & 17 & 1 & 11 \\
\hline
\end{tabular}




\begin{tabular}{|c|c|c|c|c|c|c|c|c|c|c|c|c|c|c|}
\hline Golog & Qinghai & NEC & 32 & 1 & 22 & 40 & 38 & 1 & 30 & 49 & 30 & 1 & 21 & 45 \\
\hline Haidong & Qinghai & NEC & 31 & 2 & 19 & 45 & 37 & 2 & 24 & 60 & 35 & 1 & 24 & 47 \\
\hline Xining & Qinghai & NEC & 28 & 1 & 17 & 38 & 28 & 1 & 20 & 42 & 31 & 1 & 19 & 47 \\
\hline Ali & Tibet & NEC & 3 & 0 & 2 & 5 & 4 & 0 & 3 & 7 & 6 & 1 & 2 & 11 \\
\hline Qamdo & Tibet & NEC & 16 & 1 & 11 & 32 & 14 & 1 & 10 & 24 & 16 & 1 & 6 & 27 \\
\hline Lhasa & Tibet & NEC & 20 & 1 & 13 & 32 & 18 & 1 & 12 & 23 & 18 & 1 & 10 & 29 \\
\hline Nyingchi & Tibet & NEC & 6 & 0 & 3 & 8 & 6 & 0 & 4 & 8 & 6 & 0 & 3 & 14 \\
\hline Naqu & Tibet & NEC & 12 & 0 & 9 & 16 & 14 & 1 & 10 & 19 & 15 & 1 & 10 & 26 \\
\hline Shigatse & Tibet & NEC & 15 & 3 & 7 & 52 & 11 & 0 & 9 & 14 & 10 & 1 & 5 & 18 \\
\hline Lhoka & Tibet & NEC & 8 & 0 & 3 & 10 & 9 & 1 & 5 & 14 & 8 & 0 & 4 & 13 \\
\hline
\end{tabular}

${ }^{\mathrm{a}}$ EC and NEC denote emission control and non-emission control regions, respectively, of which the latter means regions without implementation 147 of emission control measures.

148 b The pre-Parade Blue, Parade Blue, and post-Parade Blue periods indicate the periods of 1-19 August, 20 August-3 September, and 4-30 
158 Table S5. Summary of daily average $\mathrm{SO}_{2}$ concentrations $\left(\mu \mathrm{g} \mathrm{m}^{-3}\right)$ during the pre-Parade Blue, Parade Blue and post-Parade Blue periods in the 159291 cities across China.

\begin{tabular}{|c|c|c|c|c|c|c|c|c|c|c|c|c|c|c|}
\hline \multirow{2}{*}{ City } & \multirow{2}{*}{ Province } & \multirow{2}{*}{ Region $^{a}$} & \multicolumn{4}{|c|}{ Pre-Parade Blue period ${ }^{\mathrm{b}}$} & \multicolumn{4}{|c|}{ Parade Blue period $^{\mathrm{b}}$} & \multicolumn{4}{|c|}{ Post-Parade Blue period } \\
\hline & & & Mean & SE & Min & Max & Mean & SE & Min & Max & Mean & SE & Min & Max \\
\hline Beijing & Municipality & EC & 3 & 0 & 2 & 7 & 2 & 0 & 2 & 3 & 5 & 1 & 2 & 15 \\
\hline Tianjing & Municipality & $\mathrm{EC}$ & 10 & 1 & 4 & 16 & 10 & 1 & 4 & 23 & 13 & 1 & 4 & 27 \\
\hline Baoding & Hebei & $\mathrm{EC}$ & 17 & 1 & 11 & 25 & 10 & 1 & 6 & 16 & 15 & 1 & 8 & 27 \\
\hline Cangzhou & Hebei & EC & 3 & 0 & 2 & 7 & 2 & 0 & 2 & 3 & 32 & 3 & 11 & 80 \\
\hline Chengde & Hebei & EC & 9 & 1 & 5 & 18 & 6 & 1 & 3 & 18 & 8 & 1 & 3 & 20 \\
\hline Handan & Hebei & $\mathrm{EC}$ & 28 & 2 & 16 & 48 & 34 & 5 & 9 & 62 & 25 & 2 & 8 & 58 \\
\hline Hengshui & Hebei & $\mathrm{EC}$ & 17 & 1 & 10 & 23 & 12 & 1 & 3 & 20 & 22 & 2 & 7 & 52 \\
\hline Langfang & Hebei & $\mathrm{EC}$ & 9 & 1 & 4 & 19 & 4 & 0 & 2 & 8 & 10 & 2 & 2 & 40 \\
\hline Qinhuangdao & Hebei & EC & 32 & 3 & 17 & 48 & 18 & 2 & 10 & 46 & 18 & 2 & 10 & 46 \\
\hline Shijiazhuang & Hebei & EC & 19 & 2 & 7 & 39 & 13 & 1 & 6 & 19 & 30 & 3 & 7 & 52 \\
\hline Tangshan & Hebei & EC & 29 & 3 & 12 & 64 & 29 & 4 & 13 & 68 & 39 & 3 & 16 & 73 \\
\hline Xingtai & Hebei & EC & 25 & 4 & 8 & 74 & 18 & 4 & 3 & 45 & 32 & 4 & 4 & 71 \\
\hline Zhangjiakou & Hebei & $\mathrm{EC}$ & 12 & 1 & 7 & 19 & 12 & 1 & 9 & 22 & 12 & 1 & 5 & 24 \\
\hline Changzhi & Shanxi & $\mathrm{EC}$ & 32 & 2 & 18 & 59 & 28 & 3 & 10 & 50 & 25 & 2 & 9 & 51 \\
\hline Datong & Shanxi & $\mathrm{EC}$ & 20 & 2 & 12 & 37 & 18 & 1 & 12 & 28 & 22 & 2 & 9 & 58 \\
\hline Jincheng & Shanxi & EC & 29 & 2 & 14 & 46 & 25 & 2 & 12 & 38 & 31 & 3 & 9 & 65 \\
\hline Jinzhong & Shanxi & EC & 13 & 3 & 3 & 37 & 16 & 2 & 7 & 29 & 31 & 4 & 6 & 74 \\
\hline Linfen & Shanxi & EC & 17 & 1 & 7 & 32 & 14 & 1 & 8 & 23 & 16 & 2 & 3 & 37 \\
\hline Lvliang & Shanxi & $\mathrm{EC}$ & 43 & 2 & 31 & 56 & 24 & 2 & 13 & 42 & 33 & 3 & 11 & 75 \\
\hline Shuozhou & Shanxi & EC & 39 & 3 & 18 & 60 & 37 & 3 & 17 & 52 & 27 & 2 & 11 & 45 \\
\hline Taiyuan & Shanxi & $\mathrm{EC}$ & 21 & 2 & 9 & 43 & 18 & 1 & 11 & 24 & 25 & 2 & 10 & 55 \\
\hline
\end{tabular}




\begin{tabular}{|c|c|c|c|c|c|c|c|c|c|c|c|c|c|c|}
\hline Xinzhou & Shanxi & $\mathrm{EC}$ & 41 & 3 & 23 & 58 & 32 & 1 & 21 & 39 & 36 & 3 & 13 & 69 \\
\hline Yangquan & Shanxi & EC & 24 & 2 & 7 & 36 & 21 & 2 & 7 & 35 & 30 & 3 & 7 & 79 \\
\hline Yuncheng & Shanxi & $\mathrm{EC}$ & 19 & 1 & 9 & 34 & 45 & 6 & 20 & 101 & 43 & 4 & 15 & 119 \\
\hline Binzhou & Shandong & $\mathrm{EC}$ & 30 & 4 & 9 & 87 & 26 & 3 & 11 & 60 & 38 & 3 & 14 & 89 \\
\hline Dezhou & Shandong & $\mathrm{EC}$ & 18 & 2 & 5 & 35 & 21 & 2 & 11 & 41 & 30 & 3 & 11 & 70 \\
\hline Dongying & Shandong & EC & 45 & 3 & 21 & 81 & 31 & 3 & 16 & 57 & 33 & 3 & 10 & 69 \\
\hline Jinan & Shandong & $\mathrm{EC}$ & 21 & 1 & 12 & 32 & 20 & 2 & 12 & 33 & 28 & 2 & 14 & 51 \\
\hline Jining & Shandong & $\mathrm{EC}$ & 36 & 3 & 21 & 88 & 43 & 3 & 21 & 66 & 50 & 3 & 25 & 82 \\
\hline Laiwu & Shandong & $\mathrm{EC}$ & 22 & 3 & 6 & 46 & 32 & 2 & 15 & 42 & 38 & 3 & 16 & 61 \\
\hline Liaocheng & Shandong & $\mathrm{EC}$ & 20 & 2 & 7 & 36 & 21 & 2 & 12 & 45 & 34 & 2 & 15 & 53 \\
\hline Linyi & Shandong & EC & 15 & 2 & 5 & 42 & 20 & 1 & 13 & 32 & 24 & 2 & 7 & 49 \\
\hline Qingdao & Shandong & $\mathrm{EC}$ & 17 & 1 & 12 & 28 & 20 & 1 & 14 & 33 & 20 & 1 & 13 & 27 \\
\hline Rizhao & Shandong & $\mathrm{EC}$ & 13 & 1 & 8 & 23 & 16 & 1 & 11 & 26 & 18 & 1 & 9 & 41 \\
\hline Tai'an & Shandong & $\mathrm{EC}$ & 20 & 2 & 8 & 34 & 28 & 2 & 14 & 46 & 31 & 2 & 13 & 55 \\
\hline Weihai & Shandong & EC & 10 & 1 & 3 & 20 & 13 & 1 & 8 & 18 & 14 & 1 & 6 & 29 \\
\hline Weifang & Shandong & $\mathrm{EC}$ & 19 & 2 & 11 & 38 & 27 & 3 & 13 & 62 & 26 & 2 & 13 & 68 \\
\hline Yantai & Shandong & EC & 11 & 1 & 5 & 18 & 12 & 1 & 7 & 15 & 13 & 1 & 8 & 23 \\
\hline Zaozhuang & Shandong & EC & 36 & 3 & 14 & 57 & 60 & 3 & 43 & 82 & 53 & 5 & 10 & 136 \\
\hline Zibo & Shandong & $\mathrm{EC}$ & 54 & 6 & 21 & 94 & 61 & 8 & 22 & 114 & 62 & 5 & 27 & 111 \\
\hline Alxa League & Inner Mongolia & EC & 5 & 1 & 1 & 11 & 7 & 1 & 2 & 13 & 5 & 0 & 2 & 10 \\
\hline Bayannur & Inner Mongolia & $\mathrm{EC}$ & 12 & 1 & 5 & 21 & 18 & 1 & 10 & 27 & 13 & 1 & 4 & 30 \\
\hline Baotou & Inner Mongolia & EC & 25 & 2 & 11 & 37 & 20 & 2 & 9 & 29 & 23 & 2 & 8 & 41 \\
\hline Chifeng & Inner Mongolia & $\mathrm{EC}$ & 14 & 1 & 9 & 25 & 12 & 1 & 8 & 18 & 15 & 1 & 7 & 24 \\
\hline Ordos & Inner Mongolia & $\mathrm{EC}$ & 11 & 1 & 4 & 22 & 14 & 1 & 7 & 26 & 14 & 1 & 5 & 36 \\
\hline Hohhot & Inner Mongolia & $\mathrm{EC}$ & 12 & 1 & 9 & 18 & 8 & 0 & 5 & 12 & 12 & 1 & 5 & 24 \\
\hline Hulun Buir & Inner Mongolia & EC & 2 & 0 & 1 & 4 & 3 & 0 & 1 & 7 & 3 & 0 & 2 & 8 \\
\hline
\end{tabular}




\begin{tabular}{|c|c|c|c|c|c|c|c|c|c|c|c|c|c|c|}
\hline Tongliao & Inner Mongolia & EC & 12 & 1 & 6 & 19 & 8 & 1 & 4 & 15 & 12 & 1 & 5 & 21 \\
\hline Wuhai & Inner Mongolia & EC & 48 & 7 & 6 & 122 & 34 & 7 & 4 & 95 & 57 & 11 & 3 & 178 \\
\hline Hinggan League & Inner Mongolia & EC & 5 & 0 & 2 & 9 & 3 & 0 & 2 & 6 & 7 & 1 & 3 & 17 \\
\hline Anyang & Henan & EC & 14 & 2 & 5 & 31 & 26 & 3 & 13 & 40 & 32 & 2 & 10 & 59 \\
\hline Hebi & Henan & EC & 18 & 1 & 12 & 30 & 17 & 2 & 9 & 28 & 28 & 2 & 11 & 46 \\
\hline Jiaozuo & Henan & EC & 20 & 2 & 5 & 33 & 23 & 2 & 10 & 37 & 28 & 3 & 9 & 65 \\
\hline Kaifeng & Henan & EC & 13 & 1 & 10 & 19 & 16 & 1 & 10 & 28 & 18 & 1 & 7 & 32 \\
\hline Luoyang & Henan & EC & 24 & 3 & 7 & 50 & 20 & 2 & 8 & 30 & 25 & 1 & 7 & 37 \\
\hline Luohe & Henan & EC & 13 & 1 & 7 & 30 & 16 & 1 & 12 & 21 & 28 & 3 & 11 & 84 \\
\hline Nanyang & Henan & EC & 22 & 1 & 10 & 33 & 22 & 1 & 15 & 29 & 19 & 2 & 8 & 37 \\
\hline Pingdingshan & Henan & EC & 32 & 2 & 15 & 49 & 32 & 2 & 23 & 46 & 34 & 2 & 15 & 57 \\
\hline Sanmenxia & Henan & EC & 18 & 1 & 8 & 28 & 25 & 2 & 13 & 42 & 26 & 2 & 10 & 45 \\
\hline Shangqiu & Henan & EC & 10 & 1 & 5 & 15 & 18 & 1 & 13 & 22 & 20 & 1 & 11 & 34 \\
\hline Xinyang & Henan & EC & 17 & 1 & 9 & 25 & 16 & 1 & 11 & 21 & 17 & 1 & 8 & 26 \\
\hline Zhengzhou & Henan & EC & 5 & 1 & 2 & 12 & 9 & 1 & 5 & 15 & 16 & 1 & 5 & 32 \\
\hline Zhoukou & Henan & EC & 7 & 0 & 6 & 9 & 9 & 0 & 6 & 11 & 12 & 1 & 7 & 20 \\
\hline Zhumadian & Henan & EC & 26 & 2 & 7 & 45 & 45 & 8 & 16 & 141 & 28 & 2 & 10 & 61 \\
\hline Anshan & Liaoning & NEC & 14 & 1 & 7 & 25 & 19 & 1 & 12 & 26 & 21 & 1 & 11 & 36 \\
\hline Benxi & Liaoning & NEC & 7 & 0 & 6 & 12 & 12 & 1 & 7 & 18 & 18 & 1 & 10 & 28 \\
\hline Chaoyang & Liaoning & NEC & 9 & 1 & 4 & 13 & 10 & 1 & 7 & 15 & 14 & 1 & 8 & 26 \\
\hline Dalian & Liaoning & NEC & 8 & 1 & 3 & 16 & 10 & 1 & 6 & 16 & 11 & 1 & 6 & 19 \\
\hline Dandong & Liaoning & NEC & 7 & 0 & 5 & 9 & 9 & 1 & 7 & 13 & 12 & 0 & 8 & 15 \\
\hline Fushun & Liaoning & NEC & 7 & 1 & 3 & 12 & 8 & 1 & 5 & 13 & 14 & 1 & 5 & 25 \\
\hline Fuxin & Liaoning & NEC & 29 & 3 & 12 & 64 & 30 & 2 & 22 & 49 & 32 & 2 & 15 & 60 \\
\hline Huludao & Liaoning & NEC & 20 & 3 & 7 & 56 & 29 & 2 & 20 & 43 & 28 & 2 & 10 & 52 \\
\hline Jinzhou & Liaoning & NEC & 25 & 1 & 18 & 39 & 17 & 1 & 12 & 27 & 27 & 2 & 14 & 42 \\
\hline
\end{tabular}




\begin{tabular}{|c|c|c|c|c|c|c|c|c|c|c|c|c|c|}
\hline Liaoyang & Liaoning & NEC & 8 & 1 & 4 & 13 & 13 & 1 & 8 & 22 & 18 & 1 & 7 \\
\hline Panjin & Liaoning & NEC & 12 & 1 & 6 & 21 & 18 & 1 & 13 & 27 & 18 & 1 & 9 \\
\hline Shenyang & Liaoning & NEC & 12 & 1 & 4 & 24 & 10 & 1 & 6 & 16 & 18 & 2 & 5 \\
\hline Tieling & Liaoning & NEC & 10 & 1 & 5 & 19 & 10 & 1 & 6 & 13 & 13 & 1 & 5 \\
\hline Wafangdian & Liaoning & NEC & 2 & 0 & 1 & 4 & 3 & 0 & 2 & 7 & 6 & 1 & 1 \\
\hline Yingkou & Liaoning & NEC & 11 & 1 & 6 & 16 & 11 & 1 & 8 & 18 & 14 & 1 & 7 \\
\hline Baicheng & Jilin & NEC & 6 & 0 & 4 & 12 & 6 & 0 & 2 & 9 & 11 & 1 & 5 \\
\hline Baishan & Jilin & NEC & 10 & 1 & 5 & 23 & 8 & 1 & 4 & 12 & 16 & 2 & 4 \\
\hline Changchun & Jilin & NEC & 6 & 0 & 3 & 8 & 6 & 1 & 4 & 10 & 8 & 0 & 3 \\
\hline Jilin & Jilin & NEC & 10 & 1 & 7 & 19 & 14 & 1 & 9 & 26 & 17 & 2 & 9 \\
\hline Liaoyuan & Jilin & NEC & 6 & 1 & 4 & 23 & 9 & 1 & 4 & 18 & 14 & 1 & 7 \\
\hline Siping & Jilin & NEC & 10 & 2 & 3 & 43 & 6 & 1 & 1 & 12 & 11 & 1 & 3 \\
\hline Songyuan & Jilin & NEC & 5 & 1 & 2 & 26 & 2 & 0 & 1 & 4 & 5 & 0 & 2 \\
\hline Daqing & Heilongjiang & NEC & 9 & 1 & 7 & 15 & 9 & 1 & 7 & 16 & 9 & 1 & 5 \\
\hline Daxinganling & Heilongjiang & NEC & 15 & 1 & 10 & 24 & 14 & 0 & 12 & 17 & 18 & 1 & 12 \\
\hline Harbin & Heilongjiang & NEC & 5 & 0 & 3 & 7 & 5 & 0 & 4 & 6 & 7 & 0 & 4 \\
\hline Hegang & Heilongjiang & NEC & 2 & 0 & 1 & 3 & 2 & 0 & 1 & 5 & 4 & 0 & 1 \\
\hline Heihe & Heilongjiang & NEC & 10 & 0 & 8 & 12 & 12 & 1 & 8 & 24 & 11 & 0 & 7 \\
\hline Jixi & Heilongjiang & NEC & 8 & 1 & 4 & 14 & 15 & 0 & 13 & 20 & 13 & 1 & 7 \\
\hline Jiamusi & Heilongjiang & NEC & 7 & 1 & 3 & 23 & 5 & 0 & 3 & 7 & 5 & 0 & 3 \\
\hline Mudanjiang & Heilongjiang & NEC & 7 & 0 & 5 & 10 & 8 & 0 & 5 & 10 & 6 & 0 & 3 \\
\hline Qitaihe & Heilongjiang & NEC & 18 & 0 & 16 & 21 & 17 & 1 & 14 & 29 & 15 & 0 & 9 \\
\hline Qiqihar & Heilongjiang & NEC & 13 & 2 & 8 & 32 & 5 & 1 & 1 & 11 & 4 & 0 & 2 \\
\hline Shuangyashan & Heilongjiang & NEC & 14 & 1 & 9 & 31 & 10 & 1 & 6 & 18 & 10 & 1 & 6 \\
\hline Suihua & Heilongjiang & NEC & 9 & 1 & 3 & 18 & 9 & 1 & 4 & 14 & 8 & 1 & 2 \\
\hline Ankang & Shaanxi & NEC & 11 & 1 & 3 & 23 & 11 & 1 & 6 & 16 & 14 & 1 & 8 \\
\hline
\end{tabular}




\begin{tabular}{|c|c|c|c|c|c|c|c|c|c|c|c|c|c|}
\hline Baoji & Shaanxi & NEC & 3 & 0 & 2 & 4 & 5 & 0 & 3 & 7 & 5 & 0 & 2 \\
\hline Hanzhong & Shaanxi & NEC & 7 & 0 & 3 & 10 & 10 & 1 & 3 & 13 & 7 & 0 & 3 \\
\hline Shangluo & Shaanxi & NEC & 21 & 4 & 4 & 61 & 19 & 3 & 5 & 44 & 21 & 3 & 5 \\
\hline Tongchuan & Shaanxi & NEC & 4 & 0 & 2 & 7 & 9 & 1 & 6 & 14 & 8 & 1 & 2 \\
\hline Weinan & Shaanxi & NEC & 10 & 1 & 4 & 15 & 13 & 1 & 6 & 23 & 10 & 1 & 4 \\
\hline Xi'an & Shaanxi & NEC & 7 & 0 & 2 & 10 & 13 & 1 & 8 & 18 & 12 & 1 & 5 \\
\hline Xianyang & Shaanxi & NEC & 11 & 0 & 8 & 16 & 14 & 1 & 7 & 20 & 11 & 1 & 4 \\
\hline Yan'an & Shaanxi & NEC & 7 & 0 & 6 & 9 & 10 & 0 & 8 & 12 & 8 & 0 & 6 \\
\hline Yulin & Shaanxi & NEC & 5 & 1 & 2 & 10 & 8 & 1 & 3 & 11 & 6 & 1 & 1 \\
\hline Baiying & Gansu & NEC & 24 & 5 & 3 & 97 & 71 & 12 & 14 & 161 & 38 & 5 & 3 \\
\hline Dingxi & Gansu & NEC & 8 & 2 & 3 & 33 & 7 & 1 & 3 & 10 & 5 & 0 & 3 \\
\hline Gannan & Gansu & NEC & 9 & 2 & 3 & 33 & 7 & 0 & 5 & 9 & 7 & 0 & 3 \\
\hline Jiayuguan & Gansu & NEC & 21 & 2 & 11 & 39 & 11 & 2 & 6 & 31 & 21 & 2 & 3 \\
\hline Jiuquan & Gansu & NEC & 9 & 1 & 3 & 27 & 7 & 2 & 2 & 24 & 6 & 0 & 3 \\
\hline Lanzhou & Gansu & NEC & 12 & 1 & 6 & 23 & 11 & 1 & 5 & 17 & 13 & 1 & 7 \\
\hline Linxia & Gansu & NEC & 8 & 1 & 2 & 12 & 10 & 0 & 8 & 13 & 9 & 1 & 2 \\
\hline Pingliang & Gansu & NEC & 9 & 1 & 3 & 23 & 9 & 1 & 1 & 15 & 11 & 2 & 3 \\
\hline Qingyang & Gansu & NEC & 5 & 0 & 3 & 8 & 6 & 0 & 2 & 9 & 4 & 0 & 1 \\
\hline Tianshui & Gansu & NEC & 8 & 0 & 5 & 14 & 6 & 0 & 4 & 9 & 5 & 0 & 3 \\
\hline Wuwei & Gansu & NEC & 7 & 0 & 5 & 10 & 9 & 0 & 7 & 13 & 8 & 0 & 5 \\
\hline Zhangye & Gansu & NEC & 13 & 0 & 10 & 16 & 15 & 1 & 11 & 20 & 14 & 1 & 6 \\
\hline Guyuan & Ningxia & NEC & 7 & 1 & 3 & 13 & 8 & 1 & 3 & 15 & 4 & 0 & 2 \\
\hline Shizuishan & Ningxia & NEC & 55 & 4 & 27 & 90 & 37 & 4 & 12 & 62 & 33 & 3 & 13 \\
\hline Wuzhong & Ningxia & NEC & 11 & 2 & 2 & 24 & 18 & 2 & 3 & 30 & 8 & 1 & 2 \\
\hline Yinchuan & Ningxia & NEC & 15 & 2 & 3 & 29 & 21 & 2 & 5 & 30 & 16 & 2 & 4 \\
\hline Zhongwei & Ningxia & NEC & 11 & 2 & 5 & 27 & 18 & 2 & 2 & 29 & 8 & 1 & 3 \\
\hline
\end{tabular}




\begin{tabular}{|c|c|c|c|c|c|c|c|c|c|c|c|c|c|}
\hline Aksu & Sinkiang & NEC & 6 & 1 & 1 & 13 & 5 & 1 & 2 & 15 & 5 & 1 & 1 \\
\hline Hami & Sinkiang & NEC & 6 & 1 & 1 & 16 & 5 & 1 & 1 & 14 & 5 & 1 & 1 \\
\hline Hotan & Sinkiang & NEC & 38 & 4 & 9 & 74 & 27 & 4 & 8 & 55 & 31 & 3 & 8 \\
\hline Kashgar & Sinkiang & NEC & 10 & 1 & 5 & 16 & 9 & 1 & 6 & 14 & 12 & 1 & 6 \\
\hline Karamay & Sinkiang & NEC & 7 & 0 & 4 & 11 & 9 & 1 & 4 & 20 & 7 & 0 & 5 \\
\hline Kizilsu & Sinkiang & NEC & 5 & 2 & 0 & 27 & 6 & 1 & 2 & 16 & 2 & 0 & 1 \\
\hline Shihezi & Sinkiang & NEC & 9 & 1 & 2 & 25 & 8 & 1 & 2 & 14 & 9 & 1 & 3 \\
\hline Urumchi & Sinkiang & NEC & 10 & 1 & 2 & 18 & 9 & 1 & 2 & 15 & 10 & 1 & 3 \\
\hline Wujiaqu & Sinkiang & NEC & 9 & 3 & 1 & 56 & 13 & 3 & 4 & 44 & 7 & 1 & 2 \\
\hline Yili & Sinkiang & NEC & 7 & 0 & 4 & 9 & 7 & 0 & 4 & 10 & 7 & 1 & 2 \\
\hline Ezhou & Hubei & NEC & 16 & 2 & 5 & 34 & 15 & 1 & 9 & 25 & 21 & 2 & 6 \\
\hline Huanggang & Hubei & NEC & 10 & 2 & 1 & 29 & 7 & 1 & 3 & 15 & 18 & 3 & 2 \\
\hline Jingmen & Hubei & NEC & 14 & 1 & 7 & 27 & 17 & 1 & 10 & 21 & 19 & 2 & 6 \\
\hline Shiyan & Hubei & NEC & 18 & 1 & 10 & 23 & 22 & 1 & 15 & 33 & 18 & 1 & 10 \\
\hline Wuhan & Hubei & NEC & 12 & 1 & 8 & 18 & 14 & 1 & 9 & 18 & 17 & 1 & 11 \\
\hline Xianning & Hubei & NEC & 14 & 1 & 9 & 22 & 14 & 1 & 10 & 19 & 12 & 1 & 5 \\
\hline Xiangyang & Hubei & NEC & 10 & 1 & 3 & 20 & 12 & 1 & 7 & 18 & 14 & 1 & 6 \\
\hline Xiaogan & Hubei & NEC & 5 & 1 & 1 & 23 & 6 & 1 & 2 & 19 & 6 & 1 & 0 \\
\hline Yichang & Hubei & NEC & 9 & 1 & 5 & 24 & 11 & 1 & 8 & 19 & 10 & 0 & 6 \\
\hline Anqing & Anhui & NEC & 14 & 1 & 10 & 18 & 15 & 0 & 13 & 17 & 18 & 1 & 11 \\
\hline Bengbu & Anhui & NEC & 14 & 1 & 5 & 23 & 18 & 1 & 14 & 23 & 22 & 1 & 13 \\
\hline Bozhou & Anhui & NEC & 40 & 2 & 27 & 59 & 40 & 3 & 15 & 61 & 34 & 2 & 14 \\
\hline Chuzhou & Anhui & NEC & 16 & 1 & 12 & 22 & 12 & 1 & 6 & 18 & 11 & 1 & 6 \\
\hline Fuyang & Anhui & NEC & 16 & 1 & 13 & 23 & 19 & 1 & 14 & 34 & 20 & 1 & 15 \\
\hline Hefei & Anhui & NEC & 8 & 1 & 6 & 14 & 7 & 0 & 4 & 9 & 9 & 1 & 5 \\
\hline Huaibei & Anhui & NEC & 28 & 2 & 18 & 48 & 25 & 1 & 16 & 34 & 27 & 3 & 14 \\
\hline
\end{tabular}




\begin{tabular}{|c|c|c|c|c|c|c|c|c|c|c|c|c|c|c|}
\hline Huainan & Anhui & NEC & 9 & 1 & 5 & 16 & 9 & 0 & 6 & 12 & 11 & 0 & 6 & 15 \\
\hline Huangshan & Anhui & NEC & 7 & 0 & 5 & 8 & 8 & 0 & 7 & 9 & 8 & 0 & 7 & 11 \\
\hline Lu'an & Anhui & NEC & 21 & 5 & 5 & 68 & 10 & 0 & 9 & 13 & 12 & 0 & 9 & 16 \\
\hline Ma'anshan & Anhui & NEC & 14 & 2 & 5 & 31 & 19 & 2 & 11 & 29 & 14 & 2 & 5 & 41 \\
\hline Suzhou & Anhui & NEC & 9 & 1 & 5 & 14 & 11 & 1 & 7 & 18 & 14 & 1 & 9 & 22 \\
\hline Tongling & Anhui & NEC & 29 & 3 & 10 & 69 & 36 & 3 & 18 & 64 & 28 & 1 & 12 & 42 \\
\hline Wuhu & Anhui & NEC & 22 & 1 & 17 & 31 & 18 & 1 & 14 & 24 & 19 & 1 & 14 & 28 \\
\hline Changzhou & Jiangsu & NEC & 22 & 2 & 10 & 36 & 23 & 2 & 10 & 36 & 24 & 2 & 12 & 47 \\
\hline Huaian & Jiangsu & NEC & 20 & 2 & 8 & 41 & 13 & 1 & 8 & 18 & 14 & 1 & 5 & 27 \\
\hline Lianyungang & Jiangsu & NEC & 12 & 1 & 5 & 21 & 19 & 1 & 12 & 27 & 22 & 1 & 10 & 36 \\
\hline Nanjing & Jiangsu & NEC & 14 & 2 & 5 & 26 & 12 & 1 & 7 & 18 & 14 & 1 & 8 & 25 \\
\hline Nantong & Jiangsu & NEC & 25 & 2 & 12 & 43 & 25 & 3 & 9 & 46 & 23 & 1 & 14 & 44 \\
\hline Suzhou & Jiangsu & NEC & 14 & 1 & 6 & 21 & 18 & 2 & 9 & 39 & 17 & 1 & 8 & 29 \\
\hline Suqian & Jiangsu & NEC & 12 & 2 & 3 & 35 & 15 & 1 & 5 & 24 & 16 & 1 & 5 & 28 \\
\hline Taizhou & Jiangsu & NEC & 17 & 1 & 11 & 28 & 18 & 1 & 12 & 24 & 19 & 1 & 12 & 29 \\
\hline Wuxi & Jiangsu & NEC & 19 & 1 & 11 & 29 & 24 & 1 & 16 & 36 & 27 & 1 & 14 & 41 \\
\hline Xuzhou & Jiangsu & NEC & 26 & 1 & 20 & 41 & 34 & 3 & 18 & 57 & 29 & 2 & 17 & 52 \\
\hline Yanchen & Jiangsu & NEC & 14 & 2 & 5 & 27 & 14 & 1 & 10 & 18 & 17 & 1 & 7 & 26 \\
\hline Yangzhou & Jiangsu & NEC & 25 & 3 & 8 & 58 & 19 & 1 & 13 & 23 & 17 & 1 & 8 & 28 \\
\hline Zhenjiang & Jiangsu & NEC & 22 & 2 & 10 & 35 & 19 & 1 & 10 & 25 & 20 & 2 & 10 & 41 \\
\hline Shanghai & Municipality & NEC & 11 & 1 & 6 & 17 & 13 & 1 & 8 & 24 & 11 & 1 & 7 & 18 \\
\hline Hangzhou & Zhejiang & NEC & 11 & 1 & 6 & 17 & 11 & 1 & 7 & 19 & 14 & 1 & 7 & 20 \\
\hline Huzhou & Zhejiang & NEC & 10 & 1 & 4 & 18 & 14 & 2 & 4 & 31 & 12 & 1 & 4 & 20 \\
\hline Jiaxing & Zhejiang & NEC & 11 & 1 & 3 & 19 & 18 & 2 & 8 & 34 & 16 & 1 & 3 & 32 \\
\hline Jinhua & Zhejiang & NEC & 15 & 2 & 3 & 32 & 16 & 1 & 9 & 26 & 18 & 1 & 8 & 37 \\
\hline Lishui & Zhejiang & NEC & 6 & 1 & 1 & 12 & 10 & 1 & 2 & 21 & 7 & 1 & 2 & 22 \\
\hline
\end{tabular}




\begin{tabular}{|c|c|c|c|c|c|c|c|c|c|c|c|c|c|}
\hline Ningbo & Zhejiang & NEC & 10 & 1 & 6 & 16 & 13 & 1 & 9 & 20 & 14 & 1 & 7 \\
\hline Quzhou & Zhejiang & NEC & 15 & 2 & 3 & 32 & 18 & 2 & 9 & 34 & 17 & 1 & 8 \\
\hline Shaoxing & Zhejiang & NEC & 13 & 1 & 6 & 24 & 23 & 2 & 14 & 37 & 28 & 2 & 14 \\
\hline Taizhou & Zhejiang & NEC & 6 & 1 & 1 & 11 & 6 & 1 & 3 & 11 & 7 & 0 & 3 \\
\hline Wenjiang & Zhejiang & NEC & 8 & 1 & 2 & 12 & 9 & 1 & 5 & 13 & 8 & 1 & 2 \\
\hline Zhoushan & Zhejiang & NEC & 5 & 1 & 2 & 15 & 6 & 0 & 3 & 9 & 7 & 0 & 5 \\
\hline Fuzhou & Jiangxi & NEC & 14 & 1 & 3 & 23 & 18 & 3 & 6 & 37 & 18 & 2 & 7 \\
\hline Ganzhou & Jiangxi & NEC & 17 & 2 & 10 & 33 & 20 & 2 & 10 & 37 & 26 & 2 & 13 \\
\hline Ji'an & Jiangxi & NEC & 23 & 3 & 8 & 43 & 24 & 2 & 11 & 40 & 30 & 2 & 6 \\
\hline Jingdezhen & Jiangxi & NEC & 7 & 1 & 2 & 17 & 10 & 1 & 2 & 20 & 8 & 0 & 4 \\
\hline Jiujiang & Jiangxi & NEC & 40 & 1 & 27 & 52 & 39 & 2 & 29 & 49 & 37 & 1 & 26 \\
\hline Nanchang & Jiangxi & NEC & 26 & 2 & 12 & 50 & 26 & 1 & 20 & 33 & 24 & 1 & 8 \\
\hline Pingxiang & Jiangxi & NEC & 20 & 2 & 12 & 47 & 25 & 2 & 14 & 33 & 22 & 2 & 10 \\
\hline Shangrao & Jiangxi & NEC & 44 & 4 & 10 & 72 & 44 & 5 & 20 & 99 & 33 & 2 & 14 \\
\hline Xinyu & Jiangxi & NEC & 25 & 3 & 5 & 53 & 22 & 2 & 8 & 30 & 19 & 1 & 6 \\
\hline Yichun & Jiangxi & NEC & 11 & 1 & 5 & 21 & 15 & 1 & 9 & 19 & 17 & 1 & 8 \\
\hline Yingtan & Jiangxi & NEC & 21 & 2 & 13 & 41 & 26 & 3 & 15 & 48 & 37 & 3 & 14 \\
\hline Changsha & Hunan & NEC & 14 & 1 & 8 & 20 & 14 & 1 & 7 & 20 & 13 & 1 & 4 \\
\hline Changde & Hunan & NEC & 17 & 1 & 6 & 22 & 14 & 1 & 10 & 20 & 14 & 1 & 5 \\
\hline Chenzhou & Hunan & NEC & 11 & 1 & 7 & 19 & 17 & 2 & 9 & 35 & 17 & 1 & 8 \\
\hline Huaihua & Hunan & NEC & 11 & 1 & 4 & 24 & 13 & 1 & 6 & 22 & 13 & 1 & 3 \\
\hline Loudi & Hunan & NEC & 29 & 1 & 16 & 37 & 33 & 1 & 26 & 42 & 29 & 1 & 15 \\
\hline Xiangtan & Hunan & NEC & 14 & 1 & 6 & 22 & 16 & 1 & 9 & 29 & 20 & 2 & 7 \\
\hline Yiyang & Hunan & NEC & 17 & 1 & 8 & 25 & 23 & 1 & 17 & 31 & 24 & 1 & 13 \\
\hline Yongzhou & Hunan & NEC & 39 & 2 & 17 & 57 & 27 & 2 & 9 & 41 & 31 & 2 & 12 \\
\hline Zhangjiajie & Hunan & NEC & 6 & 0 & 4 & 9 & 6 & 0 & 4 & 7 & 6 & 0 & 2 \\
\hline
\end{tabular}




\begin{tabular}{|c|c|c|c|c|c|c|c|c|c|c|c|c|c|c|}
\hline Zhuzhou & Hunan & NEC & 17 & 2 & 8 & 36 & 18 & 1 & 7 & 27 & 19 & 1 & 10 & 28 \\
\hline Dongguan & Guangdong & NEC & 11 & 1 & 6 & 19 & 11 & 1 & 7 & 17 & 13 & 1 & 7 & 20 \\
\hline Foshan & Guangdong & NEC & 16 & 1 & 11 & 22 & 17 & 1 & 9 & 26 & 14 & 1 & 7 & 21 \\
\hline Guangzhou & Guangdong & NEC & 12 & 0 & 8 & 17 & 11 & 1 & 6 & 17 & 10 & 1 & 5 & 16 \\
\hline Heyuan & Guangdong & NEC & 7 & 0 & 4 & 12 & 7 & 1 & 3 & 13 & 8 & 0 & 4 & 13 \\
\hline Huizhou & Guangdong & NEC & 10 & 1 & 7 & 14 & 9 & 1 & 7 & 16 & 9 & 0 & 6 & 15 \\
\hline Jiangmen & Guangdong & NEC & 12 & 1 & 9 & 17 & 18 & 1 & 8 & 28 & 15 & 1 & 10 & 24 \\
\hline Maoming & Guangdong & NEC & 12 & 2 & 5 & 33 & 16 & 2 & 9 & 30 & 10 & 1 & 2 & 20 \\
\hline Meizhou & Guangdong & NEC & 7 & 0 & 5 & 9 & 6 & 0 & 4 & 8 & 8 & 0 & 5 & 11 \\
\hline Qingyuan & Guangdong & NEC & 18 & 1 & 12 & 25 & 10 & 1 & 5 & 15 & 12 & 1 & 6 & 22 \\
\hline Shantou & Guangdong & NEC & 14 & 1 & 9 & 18 & 13 & 1 & 8 & 18 & 12 & 1 & 8 & 23 \\
\hline Shaoguan & Guangdong & NEC & 19 & 1 & 11 & 28 & 16 & 1 & 9 & 24 & 16 & 1 & 7 & 24 \\
\hline Shenzhen & Guangdong & NEC & 9 & 0 & 6 & 13 & 8 & 1 & 6 & 12 & 7 & 0 & 5 & 13 \\
\hline Zhanjiang & Guangdong & NEC & 9 & 1 & 5 & 14 & 6 & 1 & 2 & 9 & 5 & 1 & 1 & 10 \\
\hline Zhaoqing & Guangdong & NEC & 19 & 1 & 10 & 28 & 17 & 2 & 9 & 28 & 21 & 1 & 12 & 34 \\
\hline Zhongshan & Guangdong & NEC & 7 & 1 & 4 & 17 & 8 & 1 & 4 & 17 & 9 & 1 & 4 & 19 \\
\hline Zhuhai & Guangdong & NEC & 5 & 1 & 2 & 14 & 8 & 1 & 3 & 15 & 7 & 1 & 3 & 17 \\
\hline Fuzhou & Fujian & NEC & 5 & 0 & 3 & 6 & 4 & 0 & 3 & 6 & 4 & 0 & 2 & 5 \\
\hline Longyan & Fujian & NEC & 7 & 1 & 3 & 12 & 6 & 0 & 4 & 9 & 9 & 1 & 5 & 16 \\
\hline Nanping & Fujian & NEC & 12 & 0 & 8 & 15 & 15 & 1 & 11 & 21 & 15 & 1 & 11 & 27 \\
\hline Ningde & Fujian & NEC & 2 & 0 & 1 & 5 & 3 & 0 & 1 & 5 & 3 & 0 & 1 & 7 \\
\hline Putian & Fujian & NEC & 5 & 1 & 2 & 11 & 4 & 0 & 2 & 7 & 5 & 0 & 3 & 8 \\
\hline Quanzhou & Fujian & NEC & 13 & 1 & 3 & 25 & 9 & 1 & 6 & 17 & 9 & 1 & 4 & 24 \\
\hline Sanming & Fujian & NEC & 13 & 1 & 4 & 19 & 10 & 1 & 4 & 19 & 12 & 1 & 6 & 23 \\
\hline Xiamen & Fujian & NEC & 9 & 1 & 3 & 16 & 6 & 1 & 2 & 12 & 6 & 0 & 2 & 9 \\
\hline Zhangzhou & Fujian & NEC & 9 & 1 & 5 & 16 & 11 & 1 & 7 & 19 & 11 & 1 & 5 & 17 \\
\hline
\end{tabular}




\begin{tabular}{|c|c|c|c|c|c|c|c|c|c|c|c|c|c|}
\hline Haikou & Hainan & NEC & 5 & 0 & 3 & 10 & 5 & 0 & 3 & 8 & 4 & 0 & 2 \\
\hline Sanya & Hainan & NEC & 3 & 0 & 2 & 7 & 2 & 0 & 2 & 3 & 3 & 0 & 2 \\
\hline Chongqing & Municipality & NEC & 10 & 1 & 4 & 16 & 12 & 1 & 7 & 16 & 10 & 1 & 4 \\
\hline Bazhong & Sichuan & NEC & 2 & 0 & 1 & 5 & 2 & 0 & 1 & 5 & 2 & 0 & 1 \\
\hline Chengdu & Sichuan & NEC & 13 & 1 & 7 & 20 & 15 & 1 & 7 & 24 & 12 & 1 & 6 \\
\hline Dazhou & Sichuan & NEC & 15 & 1 & 6 & 31 & 12 & 1 & 7 & 19 & 10 & 1 & 4 \\
\hline Deyang & Sichuan & NEC & 17 & 1 & 7 & 30 & 14 & 1 & 4 & 23 & 8 & 1 & 2 \\
\hline Guangyuan & Sichuan & NEC & 17 & 1 & 12 & 22 & 16 & 1 & 13 & 23 & 12 & 0 & 7 \\
\hline Leshan & Sichuan & NEC & 14 & 1 & 6 & 25 & 19 & 3 & 6 & 38 & 13 & 1 & 6 \\
\hline Luzhou & Sichuan & NEC & 12 & 1 & 6 & 20 & 15 & 2 & 6 & 27 & 11 & 1 & 5 \\
\hline Meishan & Sichuan & NEC & 15 & 1 & 8 & 26 & 13 & 2 & 2 & 30 & 8 & 1 & 2 \\
\hline Mianyang & Sichuan & NEC & 10 & 1 & 4 & 21 & 11 & 1 & 5 & 18 & 10 & 1 & 5 \\
\hline Nanchong & Sichuan & NEC & 12 & 1 & 5 & 18 & 10 & 1 & 5 & 22 & 9 & 1 & 5 \\
\hline Panzhihua & Sichuan & NEC & 23 & 2 & 12 & 44 & 30 & 2 & 19 & 44 & 35 & 2 & 16 \\
\hline Suining & Sichuan & NEC & 10 & 1 & 3 & 19 & 7 & 1 & 4 & 17 & 7 & 1 & 3 \\
\hline Ya'an & Sichuan & NEC & 14 & 2 & 6 & 56 & 8 & 0 & 6 & 10 & 10 & 0 & 6 \\
\hline Ziyang & Sichuan & NEC & 21 & 2 & 12 & 36 & 22 & 1 & 17 & 30 & 16 & 1 & 9 \\
\hline Zigong & Sichuan & NEC & 16 & 1 & 9 & 27 & 19 & 1 & 11 & 27 & 17 & 1 & 10 \\
\hline Baoshan & Yunnan & NEC & 12 & 1 & 2 & 22 & 5 & 1 & 1 & 10 & 9 & 1 & 3 \\
\hline Chuxiong & Yunnan & NEC & 23 & 2 & 12 & 42 & 27 & 3 & 12 & 56 & 19 & 2 & 10 \\
\hline Dali & Yunnan & NEC & 6 & 0 & 4 & 9 & 6 & 1 & 3 & 10 & 5 & 0 & 3 \\
\hline Dehong & Yunnan & NEC & 20 & 2 & 6 & 32 & 12 & 2 & 2 & 19 & 15 & 1 & 5 \\
\hline Honghe & Yunnan & NEC & 19 & 4 & 4 & 66 & 31 & 7 & 9 & 97 & 22 & 4 & 6 \\
\hline Kunming & Yunnan & NEC & 13 & 1 & 6 & 20 & 16 & 2 & 7 & 38 & 12 & 1 & 7 \\
\hline Lijiang & Yunnan & NEC & 11 & 1 & 5 & 17 & 8 & 0 & 6 & 11 & 8 & 0 & 3 \\
\hline Lincang & Yunnan & NEC & 17 & 3 & 4 & 50 & 18 & 3 & 3 & 43 & 22 & 2 & 11 \\
\hline
\end{tabular}




\begin{tabular}{|c|c|c|c|c|c|c|c|c|c|c|c|c|c|c|}
\hline Nujiang & Yunnan & NEC & 8 & 1 & 4 & 13 & 7 & 0 & 5 & 9 & 7 & 1 & 3 & 16 \\
\hline Qujing & Yunnan & NEC & 20 & 2 & 9 & 35 & 24 & 2 & 17 & 37 & 17 & 1 & 10 & 33 \\
\hline Wenshan & Yunnan & NEC & 10 & 1 & 3 & 23 & 8 & 1 & 4 & 14 & 7 & 1 & 2 & 20 \\
\hline Xishuangbanna & Yunnan & NEC & 10 & 1 & 6 & 19 & 8 & 0 & 6 & 10 & 8 & 0 & 2 & 12 \\
\hline Tuxi & Yunnan & NEC & 13 & 1 & 6 & 18 & 17 & 1 & 9 & 26 & 13 & 1 & 9 & 23 \\
\hline Zhaotong & Yunnan & NEC & 32 & 5 & 4 & 60 & 47 & 7 & 15 & 103 & 27 & 2 & 11 & 52 \\
\hline Anshun & Guizhou & NEC & 25 & 2 & 13 & 51 & 32 & 4 & 18 & 69 & 20 & 2 & 11 & 43 \\
\hline Bijie & Guizhou & NEC & 10 & 1 & 3 & 17 & 11 & 1 & 7 & 16 & 10 & 0 & 4 & 15 \\
\hline Guiyang & Guizhou & NEC & 7 & 0 & 5 & 13 & 8 & 1 & 4 & 12 & 7 & 0 & 5 & 12 \\
\hline Liupanshui & Guizhou & NEC & 10 & 1 & 6 & 19 & 13 & 1 & 7 & 22 & 11 & 1 & 6 & 18 \\
\hline Tongren & Guizhou & NEC & 7 & 1 & 3 & 16 & 8 & 1 & 5 & 16 & 7 & 0 & 3 & 10 \\
\hline Zunyi & Guizhou & NEC & 7 & 1 & 2 & 15 & 6 & 1 & 2 & 9 & 6 & 0 & 2 & 10 \\
\hline Baise & Guangxi & NEC & 10 & 1 & 5 & 18 & 7 & 0 & 5 & 10 & 12 & 1 & 5 & 18 \\
\hline Beihai & Guangxi & NEC & 8 & 1 & 5 & 16 & 9 & 1 & 6 & 14 & 9 & 0 & 6 & 15 \\
\hline Chongzuo & Guangxi & NEC & 8 & 1 & 5 & 14 & 14 & 2 & 5 & 24 & 10 & 1 & 4 & 21 \\
\hline Fangchenggang & Guangxi & NEC & 4 & 0 & 2 & 7 & 3 & 0 & 2 & 6 & 4 & 0 & 2 & 8 \\
\hline Guigang & Guangxi & NEC & 22 & 2 & 6 & 42 & 17 & 2 & 7 & 30 & 18 & 1 & 10 & 28 \\
\hline Guilin & Guangxi & NEC & 18 & 2 & 9 & 32 & 19 & 2 & 10 & 32 & 22 & 1 & 10 & 39 \\
\hline Hechi & Guangxi & NEC & 33 & 5 & 9 & 77 & 31 & 4 & 5 & 57 & 21 & 3 & 6 & 69 \\
\hline Hezhou & Guangxi & NEC & 15 & 1 & 11 & 22 & 20 & 1 & 13 & 30 & 16 & 1 & 8 & 23 \\
\hline Laibin & Guangxi & NEC & 25 & 4 & 6 & 68 & 14 & 2 & 5 & 32 & 20 & 2 & 7 & 54 \\
\hline Liuzhou & Guangxi & NEC & 20 & 2 & 10 & 36 & 26 & 2 & 12 & 37 & 24 & 2 & 10 & 41 \\
\hline Nanning & Guangxi & NEC & 11 & 1 & 8 & 18 & 13 & 1 & 8 & 21 & 11 & 0 & 8 & 17 \\
\hline Qinzhou & Guangxi & NEC & 13 & 1 & 8 & 18 & 14 & 1 & 8 & 21 & 14 & 1 & 9 & 24 \\
\hline Wuzhou & Guangxi & NEC & 16 & 1 & 9 & 22 & 15 & 1 & 7 & 23 & 13 & 1 & 8 & 23 \\
\hline Yulin & Guangxi & NEC & 15 & 2 & 6 & 36 & 15 & 2 & 6 & 34 & 22 & 4 & 4 & 107 \\
\hline
\end{tabular}




\begin{tabular}{|c|c|c|c|c|c|c|c|c|c|c|c|c|c|}
\hline Golog & Qinghai & NEC & 23 & 1 & 18 & 26 & 16 & 1 & 12 & 26 & 25 & 1 & 15 \\
\hline Haidong & Qinghai & NEC & 21 & 2 & 7 & 44 & 34 & 2 & 25 & 51 & 33 & 3 & 13 \\
\hline Xining & Qinghai & NEC & 17 & 2 & 7 & 38 & 26 & 2 & 9 & 37 & 18 & 2 & 5 \\
\hline Ali & Tibet & NEC & 13 & 0 & 7 & 15 & 9 & 0 & 8 & 11 & 8 & 0 & 2 \\
\hline Qamdo & Tibet & NEC & 4 & 0 & 3 & 5 & 4 & 0 & 3 & 4 & 5 & 0 & 4 \\
\hline Lhasa & Tibet & NEC & 7 & 0 & 5 & 9 & 7 & 0 & 5 & 9 & 10 & 0 & 8 \\
\hline Nyingchi & Tibet & NEC & 3 & 0 & 2 & 3 & 3 & 0 & 3 & 3 & 3 & 0 & 3 \\
\hline Naqu & Tibet & NEC & 25 & 0 & 21 & 29 & 27 & 0 & 24 & 30 & 26 & 1 & 20 \\
\hline Shigatse & Tibet & NEC & 6 & 0 & 3 & 7 & 6 & 0 & 6 & 7 & 6 & 0 & 3 \\
\hline Lhoka & Tibet & NEC & 4 & 0 & 3 & 5 & 3 & 0 & 3 & 4 & 3 & 0 & 3 \\
\hline
\end{tabular}

160

\section{5}

7

69 1

${ }^{a}$ EC and NEC denote emission control and non-emission control regions, respectively, of which the latter means regions without implementation of emission control measures.

b The pre-Parade Blue, Parade Blue, and post-Parade Blue periods indicate the periods of 1-19 August, 20 August-3 September, and 4-30 September 2015, respectively. 
172 Table S6. Summary of daily average CO concentrations ( $\mathrm{mg} \mathrm{m}^{-3}$ ) during the pre-Parade Blue, Parade Blue and post-Parade Blue periods in the 173291 cities across China.

\begin{tabular}{|c|c|c|c|c|c|c|c|c|c|c|c|c|c|c|}
\hline \multirow{2}{*}{ City } & \multirow{2}{*}{ Province } & \multirow{2}{*}{ Region $^{\mathrm{a}}$} & \multicolumn{4}{|c|}{ Pre-Parade Blue period ${ }^{\mathrm{b}}$} & \multicolumn{4}{|c|}{ Parade Blue period $^{\mathrm{b}}$} & \multicolumn{4}{|c|}{ Post-Parade Blue period ${ }^{\mathrm{b}}$} \\
\hline & & & Mean & $\mathrm{SE}$ & Min & Max & Mean & $\mathrm{SE}$ & Min & Max & Mean & $\mathrm{SE}$ & Min & Max \\
\hline Beijing & Municipality & $\mathrm{EC}$ & 0.90 & 0.06 & 0.51 & 1.42 & 0.56 & 0.04 & 0.43 & 0.94 & 0.84 & 0.06 & 0.35 & 1.48 \\
\hline Tianjing & Municipality & $\mathrm{EC}$ & 0.97 & 0.05 & 0.67 & 1.40 & 1.04 & 0.08 & 0.61 & 1.79 & 0.89 & 0.06 & 0.39 & 1.88 \\
\hline Baoding & Hebei & $\mathrm{EC}$ & 1.01 & 0.07 & 0.51 & 1.74 & 0.64 & 0.05 & 0.34 & 1.08 & 0.81 & 0.06 & 0.26 & 1.39 \\
\hline Cangzhou & Hebei & $\mathrm{EC}$ & 0.90 & 0.06 & 0.51 & 1.42 & 0.56 & 0.04 & 0.43 & 0.94 & 0.94 & 0.07 & 0.39 & 1.98 \\
\hline Chengde & Hebei & $\mathrm{EC}$ & 0.78 & 0.04 & 0.48 & 1.04 & 0.49 & 0.02 & 0.38 & 0.75 & 0.65 & 0.05 & 0.30 & 1.25 \\
\hline Handan & Hebei & $\mathrm{EC}$ & 0.99 & 0.07 & 0.48 & 1.51 & 1.51 & 0.23 & 0.67 & 3.55 & 1.20 & 0.13 & 0.58 & 3.63 \\
\hline Hengshui & Hebei & $\mathrm{EC}$ & 1.09 & 0.03 & 0.85 & 1.33 & 1.00 & 0.04 & 0.74 & 1.36 & 1.37 & 0.06 & 0.78 & 1.96 \\
\hline Langfang & Hebei & $\mathrm{EC}$ & 1.13 & 0.08 & 0.65 & 1.92 & 0.79 & 0.10 & 0.38 & 1.81 & 1.08 & 0.08 & 0.25 & 1.74 \\
\hline Qinhuangdao & Hebei & $\mathrm{EC}$ & 1.38 & 0.15 & 0.38 & 2.46 & 0.63 & 0.10 & 0.36 & 1.87 & 1.16 & 0.13 & 0.35 & 2.45 \\
\hline Shijiazhuang & Hebei & $\mathrm{EC}$ & 0.66 & 0.05 & 0.36 & 1.17 & 0.57 & 0.05 & 0.24 & 0.95 & 1.03 & 0.08 & 0.40 & 1.96 \\
\hline Tangshan & Hebei & $\mathrm{EC}$ & 1.81 & 0.21 & 0.84 & 4.53 & 1.89 & 0.23 & 0.59 & 3.91 & 1.99 & 0.19 & 0.49 & 4.76 \\
\hline Xingtai & Hebei & EC & 1.12 & 0.12 & 0.51 & 2.40 & 0.93 & 0.11 & 0.33 & 1.70 & 1.27 & 0.09 & 0.49 & 2.17 \\
\hline Zhangjiakou & Hebei & $\mathrm{EC}$ & 0.51 & 0.02 & 0.37 & 0.66 & 0.45 & 0.02 & 0.37 & 0.58 & 0.63 & 0.03 & 0.33 & 1.03 \\
\hline Changzhi & Shanxi & EC & 1.42 & 0.08 & 1.01 & 2.06 & 1.57 & 0.04 & 1.30 & 1.79 & 1.57 & 0.05 & 1.18 & 2.44 \\
\hline Datong & Shanxi & $\mathrm{EC}$ & 0.79 & 0.01 & 0.69 & 0.88 & 0.87 & 0.02 & 0.77 & 1.00 & 1.14 & 0.04 & 0.79 & 1.49 \\
\hline Jincheng & Shanxi & $\mathrm{EC}$ & 1.71 & 0.19 & 0.59 & 3.97 & 1.64 & 0.15 & 0.78 & 2.83 & 1.25 & 0.08 & 0.67 & 2.64 \\
\hline Jinzhong & Shanxi & EC & 0.88 & 0.05 & 0.61 & 1.31 & 0.59 & 0.03 & 0.36 & 0.82 & 1.03 & 0.05 & 0.42 & 1.45 \\
\hline Linfen & Shanxi & $\mathrm{EC}$ & 1.59 & 0.07 & 1.13 & 2.32 & 1.64 & 0.04 & 1.34 & 1.94 & 2.07 & 0.05 & 1.45 & 2.66 \\
\hline Lvliang & Shanxi & $\mathrm{EC}$ & 1.73 & 0.04 & 1.47 & 2.13 & 1.85 & 0.03 & 1.63 & 2.00 & 2.48 & 0.05 & 1.97 & 2.90 \\
\hline Shuozhou & Shanxi & $\mathrm{EC}$ & 1.26 & 0.04 & 0.84 & 1.51 & 1.11 & 0.02 & 1.01 & 1.27 & 1.24 & 0.04 & 0.88 & 1.76 \\
\hline Taiyuan & Shanxi & EC & 0.85 & 0.03 & 0.68 & 1.15 & 0.84 & 0.03 & 0.71 & 1.08 & 1.17 & 0.03 & 0.71 & 1.51 \\
\hline
\end{tabular}




\begin{tabular}{|c|c|c|c|c|c|c|c|c|c|c|c|c|c|c|}
\hline Xinzhou & Shanxi & $\mathrm{EC}$ & 1.00 & 0.03 & 0.70 & 1.31 & 1.84 & 0.43 & 0.84 & 7.71 & 1.21 & 0.05 & 0.82 & 1.73 \\
\hline Yangquan & Shanxi & EC & 0.85 & 0.03 & 0.67 & 1.14 & 0.63 & 0.02 & 0.52 & 0.85 & 0.95 & 0.05 & 0.34 & 1.50 \\
\hline Yuncheng & Shanxi & $\mathrm{EC}$ & 2.11 & 0.05 & 1.77 & 2.49 & 2.30 & 0.15 & 1.02 & 3.17 & 1.61 & 0.06 & 1.16 & 2.31 \\
\hline Binzhou & Shandong & EC & 1.71 & 0.08 & 1.17 & 2.32 & 1.45 & 0.07 & 1.12 & 2.24 & 1.46 & 0.06 & 0.88 & 2.29 \\
\hline Dezhou & Shandong & $\mathrm{EC}$ & 1.92 & 0.05 & 1.62 & 2.34 & 1.66 & 0.06 & 1.32 & 2.08 & 1.76 & 0.08 & 1.11 & 2.65 \\
\hline Dongying & Shandong & EC & 1.00 & 0.07 & 0.61 & 1.50 & 0.87 & 0.05 & 0.60 & 1.12 & 0.94 & 0.05 & 0.50 & 1.31 \\
\hline Jinan & Shandong & $\mathrm{EC}$ & 0.92 & 0.05 & 0.57 & 1.33 & 0.97 & 0.04 & 0.76 & 1.25 & 1.16 & 0.06 & 0.50 & 1.66 \\
\hline Jining & Shandong & $\mathrm{EC}$ & 0.99 & 0.04 & 0.72 & 1.26 & 0.92 & 0.04 & 0.70 & 1.19 & 0.97 & 0.05 & 0.59 & 1.50 \\
\hline Laiwu & Shandong & $\mathrm{EC}$ & 1.28 & 0.06 & 0.91 & 1.60 & 1.47 & 0.06 & 1.09 & 1.85 & 1.28 & 0.06 & 0.70 & 1.95 \\
\hline Liaocheng & Shandong & $\mathrm{EC}$ & 1.39 & 0.04 & 1.16 & 1.72 & 1.18 & 0.05 & 0.86 & 1.50 & 1.50 & 0.06 & 0.92 & 2.30 \\
\hline Linyi & Shandong & $\mathrm{EC}$ & 1.09 & 0.06 & 0.75 & 1.85 & 1.17 & 0.05 & 0.86 & 1.49 & 1.17 & 0.07 & 0.52 & 2.13 \\
\hline Qingdao & Shandong & $\mathrm{EC}$ & 0.62 & 0.04 & 0.33 & 0.99 & 0.63 & 0.03 & 0.48 & 0.91 & 0.61 & 0.03 & 0.41 & 1.07 \\
\hline Rizhao & Shandong & $\mathrm{EC}$ & 0.81 & 0.05 & 0.49 & 1.37 & 0.95 & 0.04 & 0.72 & 1.26 & 0.98 & 0.05 & 0.61 & 1.74 \\
\hline Tai'an & Shandong & $\mathrm{EC}$ & 1.45 & 0.08 & 1.13 & 2.56 & 1.24 & 0.06 & 0.86 & 1.62 & 1.09 & 0.05 & 0.65 & 1.53 \\
\hline Weihai & Shandong & EC & 0.68 & 0.05 & 0.39 & 1.37 & 0.54 & 0.02 & 0.37 & 0.67 & 0.47 & 0.02 & 0.24 & 0.73 \\
\hline Weifang & Shandong & $\mathrm{EC}$ & 0.71 & 0.06 & 0.34 & 1.19 & 0.87 & 0.07 & 0.58 & 1.35 & 0.88 & 0.06 & 0.40 & 1.65 \\
\hline Yantai & Shandong & EC & 0.70 & 0.05 & 0.41 & 1.28 & 0.49 & 0.02 & 0.34 & 0.62 & 0.56 & 0.03 & 0.35 & 0.87 \\
\hline Zaozhuang & Shandong & $\mathrm{EC}$ & 0.71 & 0.05 & 0.37 & 1.00 & 0.86 & 0.02 & 0.75 & 1.04 & 0.87 & 0.04 & 0.46 & 1.55 \\
\hline Zibo & Shandong & $\mathrm{EC}$ & 1.91 & 0.09 & 1.41 & 2.63 & 2.11 & 0.10 & 1.53 & 2.78 & 1.89 & 0.09 & 1.18 & 3.10 \\
\hline Alxa League & Inner Mongolia & $\mathrm{EC}$ & 0.71 & 0.04 & 0.43 & 1.05 & 0.75 & 0.04 & 0.57 & 1.07 & 0.69 & 0.02 & 0.52 & 0.93 \\
\hline Bayannur & Inner Mongolia & $\mathrm{EC}$ & 0.47 & 0.06 & 0.19 & 1.18 & 0.36 & 0.02 & 0.27 & 0.54 & 0.41 & 0.03 & 0.19 & 0.89 \\
\hline Baotou & Inner Mongolia & $\mathrm{EC}$ & 1.01 & 0.06 & 0.60 & 1.54 & 0.98 & 0.06 & 0.60 & 1.39 & 0.99 & 0.04 & 0.58 & 1.62 \\
\hline Chifeng & Inner Mongolia & $\mathrm{EC}$ & 0.86 & 0.09 & 0.35 & 1.51 & 0.54 & 0.03 & 0.39 & 0.78 & 0.67 & 0.05 & 0.34 & 1.42 \\
\hline Ordos & Inner Mongolia & $\mathrm{EC}$ & 0.60 & 0.03 & 0.40 & 0.88 & 0.61 & 0.01 & 0.48 & 0.72 & 0.66 & 0.03 & 0.43 & 1.01 \\
\hline Hohhot & Inner Mongolia & $\mathrm{EC}$ & 0.63 & 0.03 & 0.46 & 0.98 & 0.63 & 0.02 & 0.49 & 0.74 & 0.68 & 0.03 & 0.43 & 1.08 \\
\hline Hulun Buir & Inner Mongolia & EC & 0.31 & 0.03 & 0.15 & 0.58 & 0.21 & 0.03 & 0.05 & 0.42 & 0.23 & 0.03 & 0.08 & 0.87 \\
\hline
\end{tabular}




\begin{tabular}{|c|c|c|c|c|c|c|c|c|c|c|c|c|c|c|}
\hline Tongliao & Inner Mongolia & $\mathrm{EC}$ & 0.75 & 0.05 & 0.52 & 1.21 & 0.51 & 0.01 & 0.40 & 0.65 & 0.57 & 0.04 & 0.26 & 1.07 \\
\hline Wuhai & Inner Mongolia & $\mathrm{EC}$ & 0.70 & 0.06 & 0.31 & 1.20 & 0.64 & 0.06 & 0.37 & 1.04 & 0.80 & 0.06 & 0.24 & 1.42 \\
\hline Hinggan League & Inner Mongolia & EC & 0.69 & 0.04 & 0.51 & 1.31 & 0.49 & 0.02 & 0.40 & 0.66 & 0.57 & 0.03 & 0.40 & 1.12 \\
\hline Anyang & Henan & EC & 1.41 & 0.08 & 0.75 & 2.10 & 1.63 & 0.10 & 1.24 & 2.44 & 1.77 & 0.14 & 0.91 & 4.78 \\
\hline Hebi & Henan & $\mathrm{EC}$ & 0.99 & 0.06 & 0.66 & 1.54 & 1.18 & 0.14 & 0.52 & 2.25 & 1.25 & 0.07 & 0.47 & 1.93 \\
\hline Jiaozuo & Henan & $\mathrm{EC}$ & 1.50 & 0.05 & 1.14 & 1.79 & 1.38 & 0.09 & 0.90 & 2.00 & 1.40 & 0.05 & 0.87 & 2.13 \\
\hline Kaifeng & Henan & $\mathrm{EC}$ & 1.14 & 0.05 & 0.77 & 1.58 & 1.30 & 0.04 & 1.02 & 1.76 & 1.38 & 0.06 & 0.89 & 1.95 \\
\hline Luoyang & Henan & EC & 1.67 & 0.06 & 1.19 & 2.25 & 1.36 & 0.04 & 1.12 & 1.57 & 1.75 & 0.05 & 1.18 & 2.45 \\
\hline Luohe & Henan & EC & 0.66 & 0.04 & 0.46 & 1.00 & 0.74 & 0.03 & 0.53 & 1.00 & 0.81 & 0.04 & 0.49 & 1.42 \\
\hline Nanyang & Henan & $\mathrm{EC}$ & 0.69 & 0.06 & 0.31 & 1.22 & 0.70 & 0.02 & 0.57 & 0.90 & 1.03 & 0.05 & 0.68 & 1.71 \\
\hline Pingdingshan & Henan & $\mathrm{EC}$ & 0.90 & 0.04 & 0.56 & 1.10 & 0.87 & 0.04 & 0.64 & 1.13 & 1.02 & 0.04 & 0.65 & 1.61 \\
\hline Sanmenxia & Henan & $\mathrm{EC}$ & 1.05 & 0.03 & 0.76 & 1.27 & 1.13 & 0.02 & 0.97 & 1.28 & 1.15 & 0.03 & 0.81 & 1.40 \\
\hline Shangqiu & Henan & $\mathrm{EC}$ & 0.59 & 0.05 & 0.28 & 1.14 & 0.64 & 0.03 & 0.47 & 0.88 & 0.69 & 0.04 & 0.34 & 1.3 \\
\hline Xinyang & Henan & $\mathrm{EC}$ & 0.86 & 0.03 & 0.54 & 1.04 & 0.76 & 0.02 & 0.59 & 0.89 & 0.85 & 0.03 & 0.56 & 1.15 \\
\hline Zhengzhou & Henan & $\mathrm{EC}$ & 1.05 & 0.04 & 0.77 & 1.43 & 1.12 & 0.04 & 0.87 & 1.42 & 1.30 & 0.05 & 0.83 & 1.95 \\
\hline Zhoukou & Henan & $\mathrm{EC}$ & 1.02 & 0.05 & 0.77 & 1.47 & 1.35 & 0.04 & 1.06 & 1.55 & 1.27 & 0.05 & 0.76 & 1.66 \\
\hline Zhumadian & Henan & $\mathrm{EC}$ & 0.50 & 0.04 & 0.26 & 0.87 & 0.65 & 0.03 & 0.46 & 0.83 & 0.68 & 0.04 & 0.34 & 1.17 \\
\hline Anshan & Liaoning & NEC & 1.43 & 0.06 & 0.97 & 1.99 & 1.32 & 0.06 & 0.97 & 1.67 & 1.18 & 0.06 & 0.74 & 2.01 \\
\hline Benxi & Liaoning & NEC & 1.10 & 0.04 & 0.84 & 1.42 & 1.01 & 0.07 & 0.60 & 1.72 & 1.04 & 0.07 & 0.52 & 1.83 \\
\hline Chaoyang & Liaoning & NEC & 1.71 & 0.06 & 1.43 & 2.31 & 1.52 & 0.01 & 1.42 & 1.62 & 1.80 & 0.07 & 1.27 & 2.77 \\
\hline Dalian & Liaoning & NEC & 0.78 & 0.04 & 0.46 & 1.06 & 0.72 & 0.04 & 0.51 & 1.16 & 0.71 & 0.03 & 0.45 & 1.04 \\
\hline Dandong & Liaoning & NEC & 7.13 & 1.59 & 0.97 & 24.00 & 1.26 & 0.03 & 1.06 & 1.40 & 1.34 & 0.02 & 1.12 & 1.59 \\
\hline Fushun & Liaoning & NEC & 1.05 & 0.04 & 0.77 & 1.46 & 0.94 & 0.03 & 0.71 & 1.15 & 0.98 & 0.05 & 0.51 & 1.57 \\
\hline Fuxin & Liaoning & NEC & 0.86 & 0.08 & 0.45 & 1.51 & 0.55 & 0.03 & 0.44 & 0.88 & 0.80 & 0.05 & 0.41 & 1.4 \\
\hline Huludao & Liaoning & NEC & 1.26 & 0.11 & 0.55 & 1.89 & 0.90 & 0.07 & 0.60 & 1.80 & 1.12 & 0.08 & 0.65 & 2.0 \\
\hline Jinzhou & Liaoning & NEC & 1.17 & 0.09 & 0.68 & 1.95 & 0.65 & 0.04 & 0.47 & 1.08 & 0.94 & 0.05 & 0.55 & 1.6 \\
\hline
\end{tabular}




\begin{tabular}{|c|c|c|c|c|c|c|c|c|c|c|c|c|c|c|}
\hline Liaoyang & Liaoning & NEC & 1.07 & 0.04 & 0.75 & 1.44 & 1.14 & 0.05 & 0.89 & 1.59 & 1.06 & 0.06 & 0.54 & 1.50 \\
\hline Panjin & Liaoning & NEC & 0.89 & 0.06 & 0.50 & 1.47 & 0.68 & 0.04 & 0.54 & 1.18 & 0.79 & 0.04 & 0.53 & 1.48 \\
\hline Shenyang & Liaoning & NEC & 0.88 & 0.05 & 0.55 & 1.31 & 0.58 & 0.03 & 0.38 & 0.82 & 0.81 & 0.06 & 0.32 & 1.42 \\
\hline Tieling & Liaoning & NEC & 0.72 & 0.06 & 0.38 & 1.13 & 0.71 & 0.03 & 0.55 & 0.97 & 0.68 & 0.04 & 0.39 & 1.02 \\
\hline Wafangdian & Liaoning & NEC & 0.44 & 0.03 & 0.22 & 0.66 & 0.39 & 0.03 & 0.27 & 0.60 & 0.47 & 0.04 & 0.16 & 0.82 \\
\hline Yingkou & Liaoning & NEC & 0.79 & 0.05 & 0.38 & 1.08 & 0.71 & 0.05 & 0.41 & 1.02 & 0.82 & 0.08 & 0.31 & 1.93 \\
\hline Baicheng & Jilin & NEC & 0.39 & 0.02 & 0.26 & 0.56 & 0.39 & 0.03 & 0.20 & 0.62 & 0.41 & 0.03 & 0.24 & 1.16 \\
\hline Baishan & Jilin & NEC & 0.83 & 0.07 & 0.54 & 1.50 & 0.72 & 0.03 & 0.51 & 0.89 & 0.90 & 0.04 & 0.61 & 1.34 \\
\hline Changchun & Jilin & NEC & 0.68 & 0.03 & 0.52 & 1.08 & 0.64 & 0.04 & 0.48 & 0.91 & 0.70 & 0.04 & 0.40 & 1.10 \\
\hline Jilin & Jilin & NEC & 0.63 & 0.02 & 0.49 & 0.79 & 0.64 & 0.03 & 0.45 & 0.76 & 0.68 & 0.03 & 0.42 & 0.98 \\
\hline Liaoyuan & Jilin & NEC & 0.93 & 0.04 & 0.55 & 1.26 & 0.87 & 0.07 & 0.63 & 1.63 & 0.94 & 0.03 & 0.64 & 1.20 \\
\hline Siping & Jilin & NEC & 0.74 & 0.08 & 0.16 & 1.65 & 0.40 & 0.05 & 0.11 & 0.84 & 0.88 & 0.09 & 0.01 & 2.17 \\
\hline Songyuan & Jilin & NEC & 0.98 & 0.10 & 0.36 & 1.78 & 0.55 & 0.03 & 0.39 & 0.70 & 1.13 & 0.11 & 0.51 & 2.16 \\
\hline Daqing & Heilongjiang & NEC & 0.55 & 0.02 & 0.40 & 0.66 & 0.40 & 0.02 & 0.27 & 0.58 & 0.43 & 0.02 & 0.28 & 0.70 \\
\hline Daxinganling & Heilongjiang & NEC & 0.68 & 0.05 & 0.47 & 1.30 & 0.77 & 0.03 & 0.64 & 1.19 & 0.85 & 0.05 & 0.65 & 1.68 \\
\hline Harbin & Heilongjiang & NEC & 0.86 & 0.02 & 0.70 & 1.05 & 0.70 & 0.04 & 0.47 & 0.95 & 0.82 & 0.02 & 0.58 & 1.05 \\
\hline Hegang & Heilongjiang & NEC & 1.15 & 0.11 & 0.49 & 2.09 & 0.59 & 0.03 & 0.39 & 0.79 & 0.73 & 0.04 & 0.46 & 1.18 \\
\hline Heihe & Heilongjiang & NEC & 0.40 & 0.02 & 0.32 & 0.68 & 0.34 & 0.02 & 0.20 & 0.49 & 0.30 & 0.02 & 0.17 & 0.54 \\
\hline Jixi & Heilongjiang & NEC & 0.51 & 0.02 & 0.39 & 0.73 & 0.52 & 0.04 & 0.25 & 0.86 & 0.57 & 0.04 & 0.21 & 0.97 \\
\hline Jiamusi & Heilongjiang & NEC & 0.81 & 0.02 & 0.70 & 0.96 & 0.67 & 0.03 & 0.53 & 0.93 & 0.79 & 0.03 & 0.57 & 1.27 \\
\hline Mudanjiang & Heilongjiang & NEC & 0.45 & 0.01 & 0.36 & 0.54 & 0.42 & 0.02 & 0.30 & 0.55 & 0.46 & 0.02 & 0.32 & 0.74 \\
\hline Qitaihe & Heilongjiang & NEC & 0.41 & 0.01 & 0.28 & 0.56 & 0.42 & 0.02 & 0.27 & 0.60 & 0.33 & 0.02 & 0.15 & 0.58 \\
\hline Qiqihar & Heilongjiang & NEC & 0.61 & 0.03 & 0.37 & 0.92 & 0.44 & 0.02 & 0.35 & 0.64 & 0.54 & 0.02 & 0.35 & 0.81 \\
\hline Shuangyashan & Heilongjiang & NEC & 0.72 & 0.05 & 0.36 & 1.34 & 0.54 & 0.06 & 0.28 & 1.00 & 0.56 & 0.03 & 0.34 & 0.89 \\
\hline Suihua & Heilongjiang & NEC & 0.36 & 0.03 & 0.18 & 0.60 & 0.32 & 0.02 & 0.24 & 0.46 & 0.37 & 0.03 & 0.17 & 0.75 \\
\hline Ankang & Shaanxi & NEC & 0.56 & 0.02 & 0.39 & 0.77 & 0.63 & 0.01 & 0.57 & 0.75 & 0.76 & 0.02 & 0.51 & 0.97 \\
\hline
\end{tabular}




\begin{tabular}{|c|c|c|c|c|c|c|c|c|c|c|c|c|c|c|}
\hline Baoji & Shaanxi & NEC & 0.89 & 0.03 & 0.63 & 1.14 & 0.90 & 0.04 & 0.65 & 1.12 & 0.80 & 0.03 & 0.51 & 1.11 \\
\hline Hanzhong & Shaanxi & NEC & 1.10 & 0.04 & 0.78 & 1.43 & 1.53 & 0.03 & 1.30 & 1.72 & 0.97 & 0.06 & 0.57 & 1.85 \\
\hline Shangluo & Shaanxi & NEC & 1.06 & 0.04 & 0.80 & 1.43 & 1.51 & 0.06 & 1.17 & 1.93 & 2.09 & 0.05 & 1.61 & 2.69 \\
\hline Tongchuan & Shaanxi & NEC & 0.74 & 0.04 & 0.44 & 1.05 & 0.71 & 0.02 & 0.55 & 0.82 & 0.86 & 0.04 & 0.48 & 1.27 \\
\hline Weinan & Shaanxi & NEC & 0.61 & 0.02 & 0.49 & 0.73 & 0.70 & 0.03 & 0.53 & 0.86 & 1.01 & 0.05 & 0.64 & 1.53 \\
\hline Xi'an & Shaanxi & NEC & 1.00 & 0.02 & 0.83 & 1.19 & 1.16 & 0.03 & 0.96 & 1.45 & 1.18 & 0.04 & 0.82 & 1.65 \\
\hline Xianyang & Shaanxi & NEC & 0.84 & 0.03 & 0.56 & 0.99 & 0.89 & 0.03 & 0.60 & 1.08 & 0.94 & 0.04 & 0.68 & 1.31 \\
\hline Yan'an & Shaanxi & NEC & 1.15 & 0.06 & 0.69 & 1.48 & 1.05 & 0.03 & 0.89 & 1.37 & 1.19 & 0.04 & 0.94 & 1.60 \\
\hline Yulin & Shaanxi & NEC & 0.94 & 0.07 & 0.40 & 1.31 & 0.68 & 0.03 & 0.49 & 0.90 & 0.82 & 0.03 & 0.48 & 1.18 \\
\hline Baiying & Gansu & NEC & 0.76 & 0.04 & 0.48 & 1.06 & 0.71 & 0.02 & 0.59 & 0.83 & 0.91 & 0.04 & 0.73 & 1.38 \\
\hline Dingxi & Gansu & NEC & 0.53 & 0.04 & 0.30 & 1.02 & 0.43 & 0.03 & 0.30 & 0.64 & 0.41 & 0.02 & 0.31 & 0.67 \\
\hline Gannan & Gansu & NEC & 0.64 & 0.05 & 0.10 & 1.02 & 0.62 & 0.04 & 0.34 & 0.91 & 0.82 & 0.05 & 0.11 & 1.52 \\
\hline Jiayuguan & Gansu & NEC & 0.67 & 0.04 & 0.37 & 1.01 & 0.60 & 0.03 & 0.44 & 0.88 & 0.52 & 0.03 & 0.28 & 0.89 \\
\hline Jiuquan & Gansu & NEC & 0.55 & 0.03 & 0.23 & 0.80 & 0.56 & 0.04 & 0.31 & 0.94 & 0.60 & 0.03 & 0.40 & 1.08 \\
\hline Lanzhou & Gansu & NEC & 0.89 & 0.04 & 0.56 & 1.18 & 1.05 & 0.04 & 0.81 & 1.26 & 1.03 & 0.04 & 0.76 & 1.50 \\
\hline Linxia & Gansu & NEC & 0.76 & 0.01 & 0.67 & 0.92 & 0.82 & 0.03 & 0.71 & 0.99 & 0.84 & 0.03 & 0.61 & 1.11 \\
\hline Pingliang & Gansu & NEC & 0.71 & 0.06 & 0.36 & 1.64 & 0.80 & 0.03 & 0.65 & 0.97 & 0.64 & 0.03 & 0.31 & 0.96 \\
\hline Qingyang & Gansu & NEC & 0.78 & 0.02 & 0.65 & 0.97 & 0.87 & 0.03 & 0.75 & 1.07 & 0.85 & 0.03 & 0.59 & 1.18 \\
\hline Tianshui & Gansu & NEC & 0.46 & 0.01 & 0.38 & 0.62 & 0.51 & 0.02 & 0.37 & 0.69 & 0.57 & 0.03 & 0.34 & 0.90 \\
\hline Wuwei & Gansu & NEC & 2.68 & 0.04 & 2.37 & 3.02 & 2.56 & 0.11 & 2.16 & 3.22 & 1.20 & 0.17 & 0.33 & 2.51 \\
\hline Zhangye & Gansu & NEC & 0.42 & 0.03 & 0.28 & 0.82 & 0.41 & 0.02 & 0.29 & 0.51 & 0.57 & 0.08 & 0.26 & 2.30 \\
\hline Guyuan & Ningxia & NEC & 0.60 & 0.02 & 0.48 & 0.81 & 0.59 & 0.04 & 0.39 & 0.85 & 0.49 & 0.02 & 0.29 & 0.72 \\
\hline Shizuishan & Ningxia & NEC & 0.52 & 0.04 & 0.26 & 1.02 & 0.59 & 0.04 & 0.38 & 0.85 & 0.66 & 0.05 & 0.29 & 1.36 \\
\hline Wuzhong & Ningxia & NEC & 0.93 & 0.05 & 0.73 & 1.59 & 0.89 & 0.02 & 0.73 & 1.07 & 0.66 & 0.03 & 0.39 & 1.10 \\
\hline Yinchuan & Ningxia & NEC & 0.83 & 0.04 & 0.52 & 1.12 & 0.87 & 0.03 & 0.69 & 1.10 & 0.87 & 0.04 & 0.53 & 1.31 \\
\hline Zhongwei & Ningxia & NEC & 1.02 & 0.01 & 0.95 & 1.19 & 1.07 & 0.04 & 0.84 & 1.34 & 0.77 & 0.07 & 0.30 & 1.41 \\
\hline
\end{tabular}




\begin{tabular}{|c|c|c|c|c|c|c|c|c|c|c|c|c|c|c|}
\hline Aksu & Sinkiang & NEC & 0.80 & 0.10 & 0.37 & 1.76 & 1.32 & 0.14 & 0.36 & 2.35 & 0.88 & 0.14 & 0.00 & 2.49 \\
\hline Hami & Sinkiang & NEC & 0.97 & 0.06 & 0.58 & 1.42 & 0.71 & 0.05 & 0.40 & 1.02 & 0.88 & 0.07 & 0.36 & 1.82 \\
\hline Hotan & Sinkiang & NEC & 0.60 & 0.05 & 0.24 & 1.08 & 0.99 & 0.06 & 0.65 & 1.40 & 0.68 & 0.05 & 0.24 & 1.23 \\
\hline Kashgar & Sinkiang & NEC & 0.57 & 0.03 & 0.30 & 0.87 & 0.55 & 0.04 & 0.35 & 0.85 & 0.66 & 0.05 & 0.28 & 1.14 \\
\hline Karamay & Sinkiang & NEC & 1.12 & 0.03 & 0.88 & 1.36 & 1.21 & 0.03 & 1.06 & 1.36 & 1.28 & 0.04 & 0.98 & 1.65 \\
\hline Kizilsu & Sinkiang & NEC & 1.06 & 0.06 & 0.61 & 1.56 & 1.23 & 0.12 & 0.59 & 1.79 & 1.14 & 0.10 & 0.12 & 1.85 \\
\hline Shihezi & Sinkiang & NEC & 0.67 & 0.05 & 0.44 & 1.27 & 0.84 & 0.04 & 0.61 & 1.11 & 0.71 & 0.04 & 0.38 & 1.07 \\
\hline Urumchi & Sinkiang & NEC & 0.74 & 0.03 & 0.51 & 1.03 & 0.66 & 0.03 & 0.47 & 0.93 & 0.72 & 0.04 & 0.45 & 1.37 \\
\hline Wujiaqu & Sinkiang & NEC & 0.63 & 0.04 & 0.29 & 0.97 & 0.52 & 0.06 & 0.20 & 0.86 & 0.63 & 0.05 & 0.30 & 1.48 \\
\hline Yili & Sinkiang & NEC & 1.16 & 0.03 & 0.87 & 1.39 & 1.34 & 0.06 & 0.89 & 1.74 & 0.94 & 0.06 & 0.52 & 1.71 \\
\hline Ezhou & Hubei & NEC & 1.03 & 0.06 & 0.77 & 1.79 & 1.10 & 0.03 & 0.95 & 1.29 & 1.20 & 0.04 & 0.89 & 1.58 \\
\hline Huanggang & Hubei & NEC & 0.87 & 0.05 & 0.36 & 1.18 & 1.05 & 0.03 & 0.75 & 1.15 & 1.28 & 0.03 & 0.99 & 1.65 \\
\hline Jingmen & Hubei & NEC & 0.65 & 0.04 & 0.44 & 1.07 & 0.82 & 0.02 & 0.65 & 0.96 & 0.79 & 0.03 & 0.47 & 1.05 \\
\hline Shiyan & Hubei & NEC & 0.94 & 0.04 & 0.65 & 1.39 & 0.86 & 0.05 & 0.53 & 1.30 & 1.26 & 0.05 & 0.87 & 1.94 \\
\hline Wuhan & Hubei & NEC & 0.66 & 0.05 & 0.42 & 1.07 & 0.93 & 0.05 & 0.54 & 1.16 & 0.93 & 0.02 & 0.61 & 1.14 \\
\hline Xianning & Hubei & NEC & 1.10 & 0.05 & 0.74 & 1.48 & 1.44 & 0.03 & 1.11 & 1.63 & 1.30 & 0.03 & 1.07 & 1.60 \\
\hline Xiangyang & Hubei & NEC & 0.75 & 0.04 & 0.60 & 1.14 & 0.87 & 0.03 & 0.72 & 1.13 & 0.97 & 0.03 & 0.72 & 1.44 \\
\hline Xiaogan & Hubei & NEC & 1.40 & 0.06 & 1.11 & 1.92 & 1.72 & 0.05 & 1.40 & 2.14 & 1.41 & 0.10 & 0.54 & 2.81 \\
\hline Yichang & Hubei & NEC & 0.82 & 0.04 & 0.54 & 1.13 & 0.97 & 0.02 & 0.86 & 1.17 & 1.07 & 0.03 & 0.69 & 1.45 \\
\hline Anqing & Anhui & NEC & 0.45 & 0.05 & 0.16 & 0.78 & 0.62 & 0.03 & 0.43 & 0.81 & 0.65 & 0.03 & 0.39 & 0.93 \\
\hline Bengbu & Anhui & NEC & 0.65 & 0.04 & 0.30 & 0.92 & 0.85 & 0.03 & 0.69 & 1.10 & 0.76 & 0.04 & 0.49 & 1.36 \\
\hline Bozhou & Anhui & NEC & 0.48 & 0.04 & 0.25 & 0.78 & 0.88 & 0.04 & 0.60 & 1.15 & 0.93 & 0.04 & 0.54 & 1.45 \\
\hline Chuzhou & Anhui & NEC & 0.54 & 0.06 & 0.28 & 1.00 & 0.74 & 0.04 & 0.48 & 0.96 & 0.72 & 0.03 & 0.49 & 1.09 \\
\hline Fuyang & Anhui & NEC & 0.53 & 0.07 & 0.18 & 1.13 & 0.72 & 0.04 & 0.50 & 1.02 & 0.64 & 0.05 & 0.34 & 1.36 \\
\hline Hefei & Anhui & NEC & 0.75 & 0.04 & 0.45 & 1.06 & 0.85 & 0.04 & 0.66 & 1.15 & 0.86 & 0.02 & 0.68 & 1.15 \\
\hline Huaibei & Anhui & NEC & 0.85 & 0.05 & 0.57 & 1.24 & 1.20 & 0.03 & 1.01 & 1.44 & 0.99 & 0.05 & 0.58 & 1.53 \\
\hline
\end{tabular}




\begin{tabular}{|c|c|c|c|c|c|c|c|c|c|c|c|c|c|c|}
\hline Huainan & Anhui & NEC & 0.64 & 0.06 & 0.24 & 1.07 & 0.89 & 0.03 & 0.67 & 1.16 & 0.81 & 0.04 & 0.44 & 1.39 \\
\hline Huangshan & Anhui & NEC & 0.12 & 0.01 & 0.07 & 0.18 & 0.17 & 0.02 & 0.11 & 0.36 & 0.13 & 0.01 & 0.08 & 0.18 \\
\hline Lu'an & Anhui & NEC & 0.68 & 0.04 & 0.36 & 1.07 & 0.56 & 0.03 & 0.39 & 0.76 & 0.53 & 0.02 & 0.39 & 0.77 \\
\hline Ma'anshan & Anhui & NEC & 1.10 & 0.03 & 0.84 & 1.36 & 1.34 & 0.05 & 1.02 & 1.58 & 1.33 & 0.05 & 0.94 & 2.00 \\
\hline Suzhou & Anhui & NEC & 0.57 & 0.07 & 0.23 & 1.14 & 0.97 & 0.06 & 0.71 & 1.41 & 0.93 & 0.08 & 0.46 & 2.09 \\
\hline Tongling & Anhui & NEC & 0.82 & 0.05 & 0.53 & 1.23 & 1.05 & 0.06 & 0.76 & 1.53 & 0.97 & 0.03 & 0.67 & 1.25 \\
\hline Wuhu & Anhui & NEC & 0.65 & 0.05 & 0.33 & 1.00 & 0.81 & 0.04 & 0.63 & 1.19 & 0.72 & 0.03 & 0.50 & 1.08 \\
\hline Changzhou & Jiangsu & NEC & 0.80 & 0.03 & 0.59 & 1.15 & 0.85 & 0.06 & 0.43 & 1.18 & 0.71 & 0.03 & 0.46 & 1.19 \\
\hline Huaian & Jiangsu & NEC & 0.69 & 0.05 & 0.32 & 1.07 & 0.89 & 0.06 & 0.46 & 1.29 & 0.69 & 0.03 & 0.50 & 0.95 \\
\hline Lianyungang & Jiangsu & NEC & 0.62 & 0.04 & 0.30 & 0.96 & 0.87 & 0.05 & 0.52 & 1.24 & 0.71 & 0.04 & 0.32 & 1.11 \\
\hline Nanjing & Jiangsu & NEC & 0.69 & 0.04 & 0.40 & 0.98 & 0.84 & 0.05 & 0.50 & 1.19 & 0.73 & 0.03 & 0.55 & 1.09 \\
\hline Nantong & Jiangsu & NEC & 0.54 & 0.05 & 0.32 & 1.18 & 0.72 & 0.06 & 0.43 & 1.37 & 0.54 & 0.03 & 0.36 & 0.93 \\
\hline Suzhou & Jiangsu & NEC & 0.69 & 0.03 & 0.43 & 0.85 & 0.94 & 0.05 & 0.60 & 1.32 & 0.75 & 0.03 & 0.57 & 1.04 \\
\hline Suqian & Jiangsu & NEC & 0.64 & 0.04 & 0.33 & 0.97 & 1.01 & 0.05 & 0.68 & 1.46 & 0.90 & 0.05 & 0.46 & 1.73 \\
\hline Taizhou & Jiangsu & NEC & 0.84 & 0.05 & 0.52 & 1.30 & 0.92 & 0.04 & 0.66 & 1.16 & 0.87 & 0.03 & 0.67 & 1.25 \\
\hline Wuxi & Jiangsu & NEC & 0.84 & 0.03 & 0.66 & 1.32 & 1.18 & 0.05 & 0.96 & 1.65 & 1.07 & 0.02 & 0.83 & 1.31 \\
\hline Xuzhou & Jiangsu & NEC & 0.98 & 0.05 & 0.66 & 1.33 & 1.31 & 0.06 & 1.00 & 1.75 & 1.14 & 0.06 & 0.71 & 2.36 \\
\hline Yanchen & Jiangsu & NEC & 0.59 & 0.04 & 0.33 & 1.06 & 0.68 & 0.04 & 0.44 & 0.93 & 0.57 & 0.02 & 0.41 & 0.89 \\
\hline Yangzhou & Jiangsu & NEC & 0.62 & 0.05 & 0.26 & 1.01 & 0.60 & 0.03 & 0.39 & 0.74 & 0.70 & 0.03 & 0.35 & 0.94 \\
\hline Zhenjiang & Jiangsu & NEC & 0.74 & 0.03 & 0.47 & 1.05 & 0.80 & 0.04 & 0.52 & 1.00 & 0.66 & 0.03 & 0.50 & 1.05 \\
\hline Shanghai & Municipality & NEC & 0.65 & 0.02 & 0.50 & 0.76 & 0.89 & 0.05 & 0.67 & 1.26 & 0.67 & 0.03 & 0.48 & 1.00 \\
\hline Hangzhou & Zhejiang & NEC & 0.68 & 0.03 & 0.44 & 0.87 & 0.90 & 0.03 & 0.68 & 1.16 & 0.78 & 0.02 & 0.54 & 1.01 \\
\hline Huzhou & Zhejiang & NEC & 0.63 & 0.03 & 0.43 & 0.86 & 0.87 & 0.03 & 0.69 & 1.05 & 0.74 & 0.02 & 0.55 & 1.09 \\
\hline Jiaxing & Zhejiang & NEC & 0.56 & 0.02 & 0.39 & 0.74 & 0.76 & 0.04 & 0.47 & 1.02 & 0.56 & 0.03 & 0.30 & 0.95 \\
\hline Jinhua & Zhejiang & NEC & 0.67 & 0.03 & 0.47 & 0.85 & 0.85 & 0.07 & 0.63 & 1.45 & 0.80 & 0.02 & 0.53 & 0.99 \\
\hline Lishui & Zhejiang & NEC & 0.52 & 0.02 & 0.39 & 0.66 & 0.58 & 0.02 & 0.44 & 0.71 & 0.62 & 0.02 & 0.44 & 0.85 \\
\hline
\end{tabular}




\begin{tabular}{|c|c|c|c|c|c|c|c|c|c|c|c|c|c|c|}
\hline Ningbo & Zhejiang & NEC & 0.71 & 0.02 & 0.58 & 0.88 & 0.87 & 0.04 & 0.67 & 1.14 & 0.83 & 0.02 & 0.67 & 1.05 \\
\hline Quzhou & Zhejiang & NEC & 0.67 & 0.03 & 0.47 & 0.85 & 0.87 & 0.06 & 0.63 & 1.45 & 0.76 & 0.02 & 0.57 & 0.97 \\
\hline Shaoxing & Zhejiang & NEC & 0.64 & 0.04 & 0.42 & 0.92 & 0.86 & 0.05 & 0.54 & 1.23 & 0.79 & 0.03 & 0.53 & 1.09 \\
\hline Taizhou & Zhejiang & NEC & 0.61 & 0.02 & 0.41 & 0.82 & 0.70 & 0.04 & 0.46 & 0.88 & 0.72 & 0.02 & 0.54 & 0.94 \\
\hline Wenjiang & Zhejiang & NEC & 0.71 & 0.03 & 0.52 & 0.92 & 0.72 & 0.03 & 0.53 & 0.92 & 0.69 & 0.02 & 0.52 & 0.95 \\
\hline Zhoushan & Zhejiang & NEC & 0.50 & 0.03 & 0.34 & 0.82 & 0.64 & 0.04 & 0.42 & 0.93 & 0.55 & 0.03 & 0.38 & 0.92 \\
\hline Fuzhou & Jiangxi & NEC & 0.75 & 0.02 & 0.65 & 0.88 & 0.83 & 0.03 & 0.59 & 0.96 & 1.00 & 0.03 & 0.65 & 1.24 \\
\hline Ganzhou & Jiangxi & NEC & 1.22 & 0.02 & 1.06 & 1.32 & 1.29 & 0.04 & 1.09 & 1.53 & 1.26 & 0.02 & 1.11 & 1.57 \\
\hline Ji'an & Jiangxi & NEC & 0.69 & 0.02 & 0.52 & 0.82 & 0.89 & 0.02 & 0.75 & 1.05 & 0.90 & 0.02 & 0.70 & 1.18 \\
\hline Jingdezhen & Jiangxi & NEC & 0.59 & 0.02 & 0.41 & 0.75 & 0.75 & 0.04 & 0.52 & 1.09 & 0.62 & 0.01 & 0.46 & 0.83 \\
\hline Jiujiang & Jiangxi & NEC & 0.61 & 0.02 & 0.40 & 0.82 & 0.69 & 0.03 & 0.48 & 0.86 & 0.65 & 0.01 & 0.56 & 0.79 \\
\hline Nanchang & Jiangxi & NEC & 0.81 & 0.02 & 0.69 & 1.00 & 0.89 & 0.04 & 0.67 & 1.06 & 0.69 & 0.02 & 0.51 & 0.89 \\
\hline Pingxiang & Jiangxi & NEC & 0.60 & 0.04 & 0.40 & 1.10 & 0.95 & 0.07 & 0.58 & 1.76 & 0.77 & 0.03 & 0.55 & 1.04 \\
\hline Shangrao & Jiangxi & NEC & 0.49 & 0.04 & 0.31 & 0.74 & 0.48 & 0.04 & 0.33 & 0.81 & 0.39 & 0.02 & 0.31 & 0.69 \\
\hline Xinyu & Jiangxi & NEC & 1.38 & 0.05 & 1.01 & 1.96 & 1.36 & 0.05 & 1.07 & 1.67 & 1.27 & 0.02 & 1.10 & 1.71 \\
\hline Yichun & Jiangxi & NEC & 0.87 & 0.03 & 0.62 & 1.08 & 0.94 & 0.03 & 0.67 & 1.13 & 0.86 & 0.02 & 0.66 & 1.06 \\
\hline Yingtan & Jiangxi & NEC & 0.55 & 0.02 & 0.41 & 0.73 & 0.70 & 0.02 & 0.60 & 0.81 & 0.70 & 0.02 & 0.48 & 0.89 \\
\hline Changsha & Hunan & NEC & 0.64 & 0.02 & 0.53 & 0.78 & 0.82 & 0.03 & 0.63 & 1.02 & 0.80 & 0.02 & 0.60 & 1.00 \\
\hline Changde & Hunan & NEC & 1.15 & 0.05 & 0.60 & 1.52 & 1.16 & 0.04 & 0.87 & 1.42 & 1.26 & 0.04 & 0.91 & 1.75 \\
\hline Chenzhou & Hunan & NEC & 0.82 & 0.04 & 0.54 & 1.18 & 1.10 & 0.05 & 0.86 & 1.44 & 1.08 & 0.03 & 0.79 & 1.41 \\
\hline Huaihua & Hunan & NEC & 1.33 & 0.03 & 1.08 & 1.54 & 1.36 & 0.03 & 1.05 & 1.54 & 1.19 & 0.03 & 0.94 & 1.55 \\
\hline Loudi & Hunan & NEC & 1.20 & 0.08 & 0.65 & 1.75 & 1.76 & 0.05 & 1.49 & 2.09 & 1.28 & 0.05 & 0.70 & 1.61 \\
\hline Xiangtan & Hunan & NEC & 1.01 & 0.03 & 0.72 & 1.18 & 1.06 & 0.04 & 0.80 & 1.31 & 0.96 & 0.03 & 0.73 & 1.24 \\
\hline Yiyang & Hunan & NEC & 0.53 & 0.05 & 0.24 & 0.90 & 0.85 & 0.03 & 0.65 & 1.06 & 0.89 & 0.02 & 0.66 & 1.06 \\
\hline Yongzhou & Hunan & NEC & 0.46 & 0.02 & 0.33 & 0.62 & 0.63 & 0.03 & 0.37 & 0.74 & 0.67 & 0.03 & 0.36 & 0.96 \\
\hline Zhangjiajie & Hunan & NEC & 1.23 & 0.05 & 0.76 & 1.58 & 1.58 & 0.06 & 1.27 & 2.02 & 1.88 & 0.08 & 1.11 & 2.55 \\
\hline
\end{tabular}




\begin{tabular}{|c|c|c|c|c|c|c|c|c|c|c|c|c|c|c|}
\hline Zhuzhou & Hunan & NEC & 0.60 & 0.03 & 0.45 & 0.79 & 0.85 & 0.04 & 0.62 & 1.10 & 0.76 & 0.02 & 0.56 & 1.00 \\
\hline Dongguan & Guangdong & NEC & 0.68 & 0.02 & 0.55 & 0.95 & 0.82 & 0.01 & 0.72 & 0.94 & 0.72 & 0.02 & 0.59 & 1.02 \\
\hline Foshan & Guangdong & NEC & 0.75 & 0.02 & 0.63 & 0.96 & 0.86 & 0.02 & 0.72 & 1.00 & 0.86 & 0.02 & 0.65 & 1.07 \\
\hline Guangzhou & Guangdong & NEC & 0.83 & 0.02 & 0.62 & 0.98 & 0.90 & 0.02 & 0.75 & 0.98 & 0.88 & 0.02 & 0.66 & 1.04 \\
\hline Heyuan & Guangdong & NEC & 0.77 & 0.02 & 0.56 & 0.94 & 0.83 & 0.01 & 0.77 & 0.93 & 0.93 & 0.02 & 0.76 & 1.13 \\
\hline Huizhou & Guangdong & NEC & 0.72 & 0.02 & 0.60 & 0.86 & 0.78 & 0.03 & 0.62 & 0.97 & 0.56 & 0.02 & 0.42 & 0.77 \\
\hline Jiangmen & Guangdong & NEC & 0.78 & 0.02 & 0.62 & 0.98 & 0.99 & 0.04 & 0.80 & 1.26 & 0.89 & 0.03 & 0.66 & 1.13 \\
\hline Maoming & Guangdong & NEC & 0.79 & 0.02 & 0.67 & 0.96 & 0.93 & 0.04 & 0.73 & 1.18 & 0.77 & 0.02 & 0.55 & 1.03 \\
\hline Meizhou & Guangdong & NEC & 0.72 & 0.02 & 0.58 & 0.91 & 0.88 & 0.01 & 0.79 & 0.97 & 1.00 & 0.02 & 0.79 & 1.17 \\
\hline Qingyuan & Guangdong & NEC & 0.87 & 0.03 & 0.60 & 1.11 & 0.93 & 0.02 & 0.83 & 1.07 & 0.96 & 0.02 & 0.75 & 1.29 \\
\hline Shantou & Guangdong & NEC & 0.85 & 0.03 & 0.67 & 1.15 & 0.86 & 0.02 & 0.74 & 1.09 & 0.85 & 0.02 & 0.68 & 1.24 \\
\hline Shaoguan & Guangdong & NEC & 0.89 & 0.02 & 0.73 & 1.00 & 0.81 & 0.03 & 0.61 & 0.97 & 1.04 & 0.03 & 0.86 & 1.32 \\
\hline Shenzhen & Guangdong & NEC & 0.83 & 0.02 & 0.69 & 0.95 & 0.82 & 0.03 & 0.67 & 1.01 & 0.76 & 0.02 & 0.62 & 0.96 \\
\hline Zhujiang & Guangdong & NEC & 0.74 & 0.03 & 0.44 & 0.90 & 0.70 & 0.02 & 0.55 & 0.84 & 0.71 & 0.02 & 0.57 & 0.94 \\
\hline Zhaoqing & Guangdong & NEC & 0.62 & 0.02 & 0.50 & 0.81 & 0.84 & 0.04 & 0.55 & 1.11 & 0.87 & 0.02 & 0.66 & 1.18 \\
\hline Zhongshan & Guangdong & NEC & 0.88 & 0.03 & 0.59 & 1.11 & 1.00 & 0.07 & 0.59 & 1.28 & 0.87 & 0.03 & 0.56 & 1.20 \\
\hline Zhuhai & Guangdong & NEC & 0.72 & 0.02 & 0.53 & 0.87 & 0.83 & 0.04 & 0.60 & 1.00 & 0.74 & 0.02 & 0.56 & 0.99 \\
\hline Fuzhou & Fujian & NEC & 0.57 & 0.02 & 0.43 & 0.80 & 0.62 & 0.03 & 0.41 & 0.79 & 0.69 & 0.02 & 0.50 & 1.06 \\
\hline Longyan & Fujian & NEC & 0.68 & 0.02 & 0.47 & 0.84 & 0.71 & 0.03 & 0.54 & 0.88 & 0.85 & 0.02 & 0.65 & 1.14 \\
\hline Nanping & Fujian & NEC & 0.78 & 0.02 & 0.60 & 0.92 & 0.77 & 0.01 & 0.67 & 0.84 & 0.81 & 0.02 & 0.67 & 1.02 \\
\hline Ningde & Fujian & NEC & 0.65 & 0.04 & 0.27 & 1.08 & 0.60 & 0.05 & 0.34 & 0.85 & 0.70 & 0.03 & 0.49 & 1.22 \\
\hline Putian & Fujian & NEC & 0.63 & 0.03 & 0.46 & 0.97 & 0.59 & 0.03 & 0.38 & 0.75 & 0.61 & 0.03 & 0.38 & 1.01 \\
\hline Quanzhou & Fujian & NEC & 0.55 & 0.02 & 0.36 & 0.70 & 0.59 & 0.02 & 0.44 & 0.72 & 0.59 & 0.02 & 0.43 & 0.95 \\
\hline Sanming & Fujian & NEC & 1.17 & 0.05 & 0.82 & 1.46 & 1.04 & 0.06 & 0.74 & 1.41 & 1.09 & 0.04 & 0.80 & 1.52 \\
\hline Xiamen & Fujian & NEC & 0.44 & 0.02 & 0.31 & 0.64 & 0.56 & 0.03 & 0.33 & 0.72 & 0.60 & 0.02 & 0.45 & 0.80 \\
\hline Zhangzhou & Fujian & NEC & 0.55 & 0.02 & 0.37 & 0.71 & 0.57 & 0.02 & 0.45 & 0.64 & 0.60 & 0.02 & 0.44 & 0.85 \\
\hline
\end{tabular}




\begin{tabular}{|c|c|c|c|c|c|c|c|c|c|c|c|c|c|c|}
\hline Haikou & Hainan & NEC & 0.52 & 0.02 & 0.35 & 0.72 & 0.63 & 0.03 & 0.48 & 0.79 & 0.58 & 0.02 & 0.40 & 0.87 \\
\hline Sanya & Hainan & NEC & 0.57 & 0.02 & 0.44 & 0.75 & 0.64 & 0.04 & 0.46 & 0.89 & 0.71 & 0.02 & 0.59 & 0.88 \\
\hline Chongqing & Municipality & NEC & 0.84 & 0.04 & 0.57 & 1.12 & 1.11 & 0.03 & 0.86 & 1.28 & 1.11 & 0.02 & 0.77 & 1.35 \\
\hline Bazhong & Sichuan & NEC & 0.67 & 0.02 & 0.55 & 0.79 & 0.82 & 0.02 & 0.64 & 0.93 & 0.81 & 0.02 & 0.56 & 1.02 \\
\hline Chengdu & Sichuan & NEC & 0.84 & 0.03 & 0.52 & 1.06 & 1.07 & 0.05 & 0.79 & 1.44 & 0.96 & 0.03 & 0.77 & 1.32 \\
\hline Dazhou & Sichuan & NEC & 0.66 & 0.05 & 0.40 & 1.06 & 0.96 & 0.05 & 0.60 & 1.23 & 0.82 & 0.03 & 0.52 & 1.18 \\
\hline Deyang & Sichuan & NEC & 0.90 & 0.03 & 0.67 & 1.08 & 1.24 & 0.04 & 0.94 & 1.51 & 0.90 & 0.03 & 0.66 & 1.26 \\
\hline Guangyuan & Sichuan & NEC & 0.26 & 0.01 & 0.18 & 0.37 & 0.37 & 0.02 & 0.26 & 0.45 & 0.43 & 0.01 & 0.28 & 0.59 \\
\hline Leshan & Sichuan & NEC & 0.81 & 0.03 & 0.64 & 1.03 & 1.03 & 0.06 & 0.70 & 1.45 & 0.92 & 0.02 & 0.71 & 1.37 \\
\hline Luzhou & Sichuan & NEC & 0.52 & 0.03 & 0.32 & 0.82 & 0.66 & 0.03 & 0.42 & 0.90 & 0.47 & 0.03 & 0.18 & 0.83 \\
\hline Meishan & Sichuan & NEC & 0.46 & 0.02 & 0.29 & 0.63 & 0.67 & 0.03 & 0.43 & 0.90 & 0.55 & 0.03 & 0.34 & 0.92 \\
\hline Mianyang & Sichuan & NEC & 0.80 & 0.03 & 0.60 & 1.00 & 0.90 & 0.03 & 0.73 & 1.12 & 0.79 & 0.02 & 0.57 & 0.99 \\
\hline Nanchong & Sichuan & NEC & 0.64 & 0.03 & 0.48 & 0.92 & 0.73 & 0.02 & 0.59 & 0.88 & 0.61 & 0.02 & 0.44 & 0.81 \\
\hline Panzhihua & Sichuan & NEC & 1.43 & 0.05 & 1.12 & 1.83 & 1.47 & 0.06 & 1.13 & 1.93 & 1.34 & 0.03 & 1.02 & 1.67 \\
\hline Suining & Sichuan & NEC & 0.82 & 0.02 & 0.69 & 1.02 & 0.78 & 0.02 & 0.69 & 0.91 & 0.92 & 0.02 & 0.80 & 1.17 \\
\hline Ya'an & Sichuan & NEC & 0.65 & 0.03 & 0.50 & 0.98 & 0.74 & 0.02 & 0.56 & 0.89 & 0.77 & 0.03 & 0.53 & 1.04 \\
\hline Ziyang & Sichuan & NEC & 0.50 & 0.02 & 0.38 & 0.72 & 0.62 & 0.02 & 0.44 & 0.75 & 0.62 & 0.02 & 0.43 & 0.80 \\
\hline Zigong & Sichuan & NEC & 0.51 & 0.04 & 0.29 & 0.85 & 0.78 & 0.04 & 0.52 & 1.07 & 0.68 & 0.03 & 0.37 & 0.90 \\
\hline Baoshan & Yunnan & NEC & 0.71 & 0.02 & 0.59 & 0.94 & 0.88 & 0.04 & 0.67 & 1.16 & 0.61 & 0.04 & 0.27 & 1.03 \\
\hline Chuxiong & Yunnan & NEC & 0.70 & 0.03 & 0.53 & 0.94 & 0.83 & 0.04 & 0.57 & 1.13 & 0.70 & 0.02 & 0.55 & 0.84 \\
\hline Dali & Yunnan & NEC & 0.41 & 0.02 & 0.27 & 0.58 & 0.45 & 0.04 & 0.25 & 0.79 & 0.30 & 0.01 & 0.21 & 0.40 \\
\hline Dehong & Yunnan & NEC & 0.49 & 0.02 & 0.35 & 0.67 & 0.63 & 0.02 & 0.53 & 0.75 & 0.66 & 0.02 & 0.41 & 0.86 \\
\hline Honghe & Yunnan & NEC & 0.46 & 0.04 & 0.28 & 0.95 & 0.54 & 0.05 & 0.31 & 0.86 & 0.46 & 0.02 & 0.29 & 0.79 \\
\hline Kunming & Yunnan & NEC & 0.77 & 0.04 & 0.47 & 1.05 & 0.85 & 0.03 & 0.64 & 1.04 & 0.84 & 0.03 & 0.65 & 1.19 \\
\hline Lijiang & Yunnan & NEC & 0.98 & 0.03 & 0.71 & 1.17 & 0.84 & 0.02 & 0.72 & 0.97 & 0.75 & 0.03 & 0.41 & 1.07 \\
\hline Lincang & Yunnan & NEC & 0.30 & 0.04 & 0.11 & 0.91 & 0.49 & 0.02 & 0.36 & 0.63 & 0.58 & 0.03 & 0.39 & 1.12 \\
\hline
\end{tabular}




\begin{tabular}{|c|c|c|c|c|c|c|c|c|c|c|c|c|c|c|}
\hline Nujiang & Yunnan & NEC & 0.62 & 0.04 & 0.30 & 0.95 & 0.54 & 0.02 & 0.43 & 0.66 & 0.64 & 0.02 & 0.44 & 0.77 \\
\hline Qujing & Yunnan & NEC & 0.76 & 0.03 & 0.56 & 1.10 & 1.06 & 0.04 & 0.81 & 1.38 & 0.92 & 0.03 & 0.62 & 1.22 \\
\hline Wenshan & Yunnan & NEC & 0.57 & 0.02 & 0.40 & 0.82 & 0.58 & 0.02 & 0.49 & 0.73 & 0.50 & 0.02 & 0.31 & 0.63 \\
\hline Xishuangbanna & Yunnan & NEC & 0.59 & 0.02 & 0.47 & 0.82 & 0.64 & 0.02 & 0.54 & 0.79 & 0.64 & 0.02 & 0.47 & 0.79 \\
\hline Tuxi & Yunnan & NEC & 0.97 & 0.07 & 0.68 & 1.97 & 1.29 & 0.06 & 0.99 & 1.70 & 1.12 & 0.06 & 0.80 & 1.99 \\
\hline Zhaotong & Yunnan & NEC & 0.71 & 0.04 & 0.40 & 1.08 & 0.98 & 0.04 & 0.74 & 1.23 & 0.96 & 0.04 & 0.60 & 1.35 \\
\hline Anshun & Guizhou & NEC & 0.31 & 0.02 & 0.20 & 0.47 & 0.49 & 0.03 & 0.37 & 0.70 & 0.41 & 0.02 & 0.23 & 0.69 \\
\hline Bijie & Guizhou & NEC & 0.80 & 0.05 & 0.45 & 1.21 & 1.10 & 0.05 & 0.77 & 1.42 & 0.91 & 0.03 & 0.61 & 1.19 \\
\hline Guiyang & Guizhou & NEC & 0.53 & 0.02 & 0.39 & 0.73 & 0.71 & 0.02 & 0.63 & 0.89 & 0.62 & 0.02 & 0.41 & 0.84 \\
\hline Liupanshui & Guizhou & NEC & 0.46 & 0.06 & 0.14 & 1.30 & 0.61 & 0.04 & 0.41 & 0.98 & 0.52 & 0.03 & 0.29 & 0.85 \\
\hline Tongren & Guizhou & NEC & 0.48 & 0.02 & 0.33 & 0.68 & 0.67 & 0.01 & 0.60 & 0.72 & 0.51 & 0.02 & 0.20 & 0.73 \\
\hline Zunyi & Guizhou & NEC & 0.63 & 0.02 & 0.50 & 0.80 & 0.80 & 0.03 & 0.56 & 1.00 & 0.84 & 0.02 & 0.54 & 1.11 \\
\hline Baise & Guangxi & NEC & 1.00 & 0.03 & 0.75 & 1.20 & 0.48 & 0.03 & 0.31 & 0.78 & 0.60 & 0.02 & 0.37 & 0.88 \\
\hline Beihai & Guangxi & NEC & 0.81 & 0.03 & 0.63 & 0.99 & 1.08 & 0.03 & 0.87 & 1.25 & 1.04 & 0.02 & 0.85 & 1.23 \\
\hline Chongzuo & Guangxi & NEC & 0.76 & 0.02 & 0.62 & 0.93 & 0.87 & 0.03 & 0.68 & 1.04 & 0.90 & 0.03 & 0.67 & 1.15 \\
\hline Fangchenggang & Guangxi & NEC & 0.65 & 0.01 & 0.60 & 0.72 & 0.85 & 0.03 & 0.65 & 1.03 & 0.77 & 0.03 & 0.48 & 1.13 \\
\hline Guigang & Guangxi & NEC & 0.79 & 0.02 & 0.65 & 1.03 & 0.92 & 0.04 & 0.66 & 1.21 & 1.00 & 0.03 & 0.73 & 1.47 \\
\hline Guilin & Guangxi & NEC & 0.77 & 0.01 & 0.68 & 0.85 & 0.83 & 0.02 & 0.69 & 0.99 & 1.04 & 0.02 & 0.83 & 1.25 \\
\hline Hechi & Guangxi & NEC & 1.10 & 0.05 & 0.76 & 1.60 & 1.09 & 0.06 & 0.71 & 1.50 & 1.19 & 0.06 & 0.65 & 2.18 \\
\hline Hezhou & Guangxi & NEC & 1.06 & 0.09 & 0.60 & 2.33 & 0.84 & 0.04 & 0.43 & 1.09 & 0.94 & 0.05 & 0.56 & 1.32 \\
\hline Laibin & Guangxi & NEC & 0.82 & 0.02 & 0.65 & 0.98 & 0.94 & 0.04 & 0.76 & 1.17 & 0.93 & 0.03 & 0.67 & 1.24 \\
\hline Liuzhou & Guangxi & NEC & 0.91 & 0.02 & 0.77 & 1.11 & 0.98 & 0.03 & 0.75 & 1.15 & 1.02 & 0.03 & 0.73 & 1.36 \\
\hline Nanning & Guangxi & NEC & 0.77 & 0.02 & 0.58 & 0.96 & 0.89 & 0.03 & 0.71 & 1.02 & 0.92 & 0.02 & 0.73 & 1.13 \\
\hline Qinzhou & Guangxi & NEC & 1.16 & 0.04 & 0.87 & 1.57 & 1.11 & 0.03 & 0.90 & 1.39 & 1.01 & 0.02 & 0.75 & 1.22 \\
\hline Wuzhou & Guangxi & NEC & 0.75 & 0.02 & 0.66 & 0.94 & 0.88 & 0.06 & 0.63 & 1.33 & 0.94 & 0.03 & 0.71 & 1.26 \\
\hline Yulin & Guangxi & NEC & 0.90 & 0.04 & 0.61 & 1.23 & 0.76 & 0.02 & 0.57 & 0.89 & 0.98 & 0.03 & 0.76 & 1.29 \\
\hline
\end{tabular}




\begin{tabular}{|c|c|c|c|c|c|c|c|c|c|c|c|c|c|c|}
\hline Golog & Qinghai & NEC & 0.55 & 0.01 & 0.42 & 0.63 & 0.64 & 0.03 & 0.47 & 0.92 & 0.78 & 0.08 & 0.44 & 1.89 \\
\hline Haidong & Qinghai & NEC & 0.82 & 0.04 & 0.56 & 1.22 & 0.90 & 0.04 & 0.66 & 1.15 & 0.93 & 0.04 & 0.63 & 1.51 \\
\hline Xining & Qinghai & NEC & 1.08 & 0.04 & 0.82 & 1.54 & 1.09 & 0.05 & 0.80 & 1.46 & 1.16 & 0.05 & 0.80 & 1.82 \\
\hline Ali & Tibet & NEC & 0.35 & 0.01 & 0.23 & 0.42 & 0.20 & 0.03 & 0.13 & 0.63 & 0.33 & 0.02 & 0.20 & 0.57 \\
\hline Qamdo & Tibet & NEC & 0.80 & 0.03 & 0.53 & 1.03 & 1.13 & 0.03 & 0.92 & 1.31 & 1.39 & 0.05 & 0.95 & 2.15 \\
\hline Lhasa & Tibet & NEC & 0.57 & 0.01 & 0.46 & 0.68 & 0.50 & 0.02 & 0.41 & 0.61 & 0.64 & 0.02 & 0.44 & 0.77 \\
\hline Nyingchi & Tibet & NEC & 0.41 & 0.01 & 0.34 & 0.50 & 0.44 & 0.01 & 0.39 & 0.53 & 0.47 & 0.02 & 0.32 & 0.61 \\
\hline Naqu & Tibet & NEC & 0.96 & 0.04 & 0.60 & 1.22 & 1.41 & 0.02 & 1.30 & 1.56 & 1.20 & 0.05 & 0.76 & 1.76 \\
\hline Shigatse & Tibet & NEC & 0.40 & 0.02 & 0.27 & 0.55 & 0.34 & 0.01 & 0.29 & 0.41 & 0.38 & 0.01 & 0.23 & 0.51 \\
\hline Lhoka & Tibet & NEC & 0.48 & 0.02 & 0.31 & 0.66 & 0.64 & 0.02 & 0.49 & 0.76 & 0.40 & 0.03 & 0.27 & 0.86 \\
\hline
\end{tabular}

${ }^{\mathrm{a}} \mathrm{EC}$ and NEC denote emission control and non-emission control regions, respectively, of which the latter means regions without implementation 175 of emission control measures.

176 b The pre-Parade Blue, Parade Blue, and post-Parade Blue periods indicate the periods of 1-19 August, 20 August-3 September, and 4-30 177 September 2015, respectively. 